\title{
SUPERÁLGEBRAS DE JORDAN DE DIMENSÃO FINITA
}

Faber Alberto Gómez González

DISSERTAÇÃO APRESENTADA

$\mathrm{AO}$

INSTITUTO DE MATEMÁTICA E ESTATÍSTICA

DA

UNIVERSIDADE DE SÃO PAULO

PARA

OBTENÇÃO DO TÍTULO DE MESTRE

EM

MATEMÁTICA.

Orientador: Prof. Dr. Ivan P. Shestakov.

Durante a elaboração deste trabalho, o autor recebeu auxílio financeiro da FAPESP.

São Paulo

2004 


\section{SUPERÁLGEBRAS DE JORDAN DE DIMENSÃO FINITA}

Este exemplar corresponde à redação final da dissertação debidamente corrigida e defendida por Faber Alberto Gómez González e aprovada pela comissão julgadora.

São Paulo 19 de agosto de 2004

Comissão Julgadora:

- Prof. Dr. Ivan P. Chestakov (Orientador) IME-USP.

- Prof. Dr. Luiz Antonio Peresi IME-USP.

- Prof. Dr. Plamen Koshlukov UNICAMP. 
Dedico este trabalho a:

Uriel Gómez meu pai, Stella González minha mãe, Vanessa minha irma. e a Lina as pessoas que amo. 


\section{AGRADECIMENTOS}

Agradeço primeiramente meu orientador, professor Ivan Shestakov, pela confiança, dedicação, paciência e amizade.

Agredeço a professora Lucia. Satie Ikemoto, pela ajuda e as observações feitas.

Agradeço a minhas amigas Valeria, Regina e Carol, que corrigiram alguns dos meus muitos erros de português.

Agradeço aos professores do IME-USP, Michael Dokuchaev, Luiz Antonio Peresi, Cesar Polcino, Luiz Fishmann, pelo mundo de conhecimento que compartilharam comigo.

Agradeço a meus amigos da POLI-USP, que sempre me tiraram um sorriso quando me achava com vontade de fugir.

Agradeço a todo o pessoal do IME-USP.

Agradeço a FAPESP, por ter financiado este projeto e por ter acreditado em mim.

Agradeço a todos aqueles que acreditaram que meu sonho pode ser feito realidade.

MUITO OBRIGADO!!! 


\section{RESUMO}

Neste trabalho estudamos as superálgebras de Jordan de dimensão finita. Mais precisamente, estudamos os teoremas de classificação de superálgebras de Jordan simples de dimensão finita em corpos algebricamente fechados de característica zero e também em corpos de característica prima. 


\section{Sumário}

INTRODUÇÃO

1 NOÇÕES BÁSICAS * 4

1.1 Preliminares . . . . . . . . . . . . . . . . 4

1.2 Álgebra de multiplicação . . . . . . . . . . . . . . . . 6

1.3 Álgebras simples . . . . . . . . . . . . . . . . . . 6

1.4 Álgebras livres e variedades de álgebras . . . . . . . . . . . . . . 9

1.4 .1 Álgebras livres . . . . . . . . . . . . . . . . . 9 9

1.4 .2 Variedades . . . . . . . . . . . . . . 10

1.4 .3 Variedades homogêneas . . . . . . . . . . . . 13

1.5 Radical . . . . . . . . . . . . . . . 15

1.6 Bimódulos . . . . . . . . . . . . . . . . . . 18

2 ÁlGEBRAS DE JORDAN DE DIMENSÃO FINITA 20

2.1 Álgebras de Jordan especiais . . . . . . . . . . . . . . 21

2.1.1 Exemplos de álgebras de Jordan . . . . . . . . . 23

2.2 Envolventes multiplicativas . . . . . . . . . . . . 25

2.2 .1 Identidades em $\mathcal{R}(J) \ldots \ldots \ldots$

2.3 Álgebras de Jordan de dimensão finita . . . . . . . . . . . . . 27

2.4 Decomposição de Peirce . . . . . . . . . . . . . . . . . . . 32

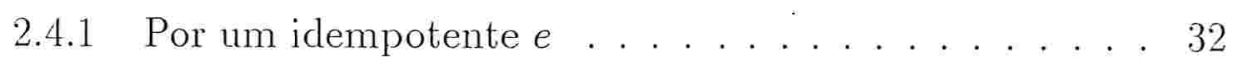


2.4.2 Por um conjunto de idempotentes . . . . . . . . . . 37

2.5 Álgebras de Jordan reduzidas . . . . . . . . . . . . . . . . 38

2.5.1 Triplo produto de Jordan e inversos . . . . . . . . . . 40

2.6 Teorema de Coordenatização . . . . . . . . . . . . . . . . 44

2.7 Álgebras de Jordan Simples . . . . . . . . . . . . . . . . 50

\section{SUPERÁLGEBRAS}

3.1 Superálgebras Simples . . . . . . . . . . . . . . . 54

3.2 Superálgebra de Grassmann . . . . . . . . . . . . . 57

3.2 .1 Álgebra de Grassmann . . . . . . . . . . . . . . . 57

3.3 Outros exemplos de superálgebras . . . . . . . . . . . . 61

3.3.1 Superálgebra de Clifford . . . . . . . . . . . . 62

3.3.2 Superálgebra $W_{m} \ldots \ldots \ldots \ldots$. . . . . . 63

3.3.3 Superálgebras de colchetes . . . . . . . . . . 63

4 SUPERÁLGEBRAS DE JORDAN 66

4.1 Em corpos de característica zero . . . . . . . . . . 67

4.2 Em corpos de característica prima $p \ldots \ldots . \ldots 78$

4.2.1 Parte par de $J=A+M$ semi-simples . . . . . . . 78

4.2.2 Parte par de $J=A+M$ não é semi-simples . . . . . . 80

4.3 Algumas observações . . . . . . . . . . . . . . . . 82 


\section{INTRODUÇÃO}

As álgebras de Jordan, eram junto com as álgebras de Lie, as primeiras álgebras não-associativas que atraíram a atenção de matemáticos. As álgebras de Jordan foram introduzidas na década dos 30 por Jordan, Von Neuman e Wigner como uma ferramenta algébrica para um formalismo da mecânica quântica, a idéia foi capturar a essência algébrica dos observáveis na mecânica quântica interpretados como operadores hermitianos no espaço de Hilbert. Dado que o produto $x y$ de dois observáveis não é observável (hermitiano), só o produto simétrico $x \circ y=x y+y x$ tem um sentido físico. Este produto de Jordan é comutativo, mas não é associativo, embora satisfaz a regra debilitada de associatividade $\left(x^{2} \circ y\right) \circ x=x^{2} \circ(y \cdot x)$ Identidade de Jordan.

A teoria de Jordan busca a descrição intrínseca desses sistemas, sem referência à hipótese-mas-não-observável produto associativo. Cada sistema abstrato com produto comutativo que satisfaz a identidade de Jordan é dito uma álgebra de Jordan. A teoria das álgebras de Jordan tornou-se um dos ramos mais importantes da Álgebra, com muitas aplicações na física teórica e na matemática.

A teoria estrutural de álgebras de Jordan de climensão finita, está bem desenvolvida, ver [2], [4]. Esta teoria tem as mesmas características das álgebras de Lie e das álgebras associativas. A existência de um ideal radical (ideal maximal nilpotente), tal que ao fazer o quociente por este ideal 
a subálgebra resultante é soma de álgebras simples. Além disso, sobre as restrições naturais, a álgebra contém uma subálgebra semi-simples isomorfa à álgebra quociente (Teorema de Wederburn).

Durante as últimas duas décadas, a teoria de superálgebras foi desenvolvida intensivamente. Superálgebras são sistemas algébricos graduados $A=A_{0} \dot{+} A_{1}$, que constam duma parte par $A_{0}$ e uma parte ímpar $A_{1}$, que se multiplicam segundo a regra $A_{i} A_{j} \subseteq A_{i+j}$ onde os subíndices são considerados módulo 2.

Uma superálgebra $J$ é dita uma superálgebra de Jordan quando a álgebra envolvente de Grassman de $J, \Gamma(J)$ é uma álgebra de Jordan. Sendo $\Gamma=\operatorname{Alg}\left\langle e_{1}, \ldots, e_{n} \ldots / e_{i}{ }^{2}=0, \forall i, e_{i} e_{j}=-e_{j} e_{i}, \forall i \neq j\right\rangle$ a. álgebra de Grasmman nas variáveis $e_{1}, \ldots, e_{n}, \ldots$ com componentes $\Gamma_{0}$ e $\Gamma_{1} . \Gamma(J)=$ $\Gamma_{0} \otimes J_{0}+\Gamma_{1} \otimes J_{1}$.

Em geral uma superálgebra de Jordan não é uma álgebra de Jordan, embora a parte par $J_{0}$ é de Jordan e a parte ímpar $J_{1}$ possui um produto do tipo Lie com valores na parte par. Esses objetos apareceram primeiro na Física das Partículas Elementares, onde as duas partes capturaram a supersimetria entre Bosones e Fermiones, mas resultaram ser importantes desde o ponto de vista puramente algébrico. Elas produziram idéias e métodos novos e permitiram resolver alguns problemas algébricos conhecidos. A teoria destas estruturas novas ainda está sendo construída e contém muitos problemas abertos.

Toda superálgebra de Jordan de dimensão finita $J$ tem um radical solúvel $S(J)$, de modo que a álgebra quociente $J / S(J)$ é semi-simples, isto é, já não possui os ideais solúveis. O radical solúvel no caso das superálgebras de Jordan a diferença do ideal radical solúvel no caso das álgebras de Jordan nem sempre é nilpotente, como foi demonstrado por Shestakov em [18]. A 
estrutura de superálgebras de Jordan semi-simples se reduz às superálgebras simples.

O estudo das superálgebras de Jordan de dimensão finita simples foi iniciado por $V$. Kac em [6], que fez uma primeira classificação destas em corpos algebricamente fechados de característica zero, empregando resultados conhecidos na classificação de superálgebras de Lie simples. Logo Kantor em [8], completou a lista. Em corpos algebricamente fechados de característica prima $p>2$, a classificação foi dada por Racine e Zelmanov em [11], que acrescentaram à lista inicial outras superálgebras sobre corpos de característica três. Finalmente, Martinez e Zelmanov em [12], estudaram as superálgebras de Jordan de dimensão finita com parte par não semi-simples.

Podemos concluir, então, que o estudo das superálgebras de Jordan tem grande importância tanto na Matemática como na Física e que o estudo das propriedades delas é um dos ramos principais em álgebra. O estudo das propriedades de superálgebras de Jordan está ainda pouco desenvolvido em comparação com a mesma teoria para as superálgebras associativas e superálgebras de Lie. Parece uma tarefa natural verificar quais resultados da teoria de álgebras de Jordan de dimensão finita admitem uma generalização para superálgebras. Em particular, sería interesante verificar a validade do Teoremade Wedderburn sobre a decomposição de uma álgebra numa soma do radical e a parte semi-simples. 


\section{Capítulo 1}

\section{NOÇÕES BÁSICAS}

\subsection{Preliminares}

Definição 1.1 Seja $V$ um espaço vetorial sobre um corpo $\mathbb{F}$. Se é possível definir em $V$ uma operação binária "o", que chamamos de multiplicação, tal que para todo $u, v, w \in V$ e para todo $\alpha \in \mathbb{F}$ se cumpre:

1. $v \circ(w+u)=v \circ w+v \circ u$

2. $(w+u) \circ v=w \circ v+u \circ v$

3. $\alpha(v \circ w)=(\alpha v) \circ w=v \circ(\alpha w)$

Então, dizemos que $(V, \circ)$ determina uma álgebra. Se a operaçẫo "o" é associativa, isto é $(v \circ w) \circ u=v \circ(w \circ u)$, então dizemos que a álgebra $V$ é uma álgebra associativa.

\section{Exemplo 1.2}

1. Todo corpo é uma álgebra sobre se mesmo.

2. Seja $V$ um espaço vetorial sobre $\mathbb{F}$, End $V$ é uma álgebra sobre $\mathbb{F}$ com multiplicação dada pela composição de aplicações. 
3. Seja $V=\left\{\sum_{i, j} \alpha_{i j} x_{i} x_{j}, \alpha_{i j} \in \mathbb{F}\right\}$ o espaço das formas quadráticas, com multiplicação $f \cdot g=\sum_{i j} \frac{\partial f}{\partial x_{i}} \frac{\partial g}{\partial x_{j}}$, é uma álgebra sobre $\mathbb{F}$.

4. $\mathbb{R}^{3}$ com multiplicação dada pelo produto vetorial é uma álgebra sobre $\mathbb{R}$.

A álgebra no item 1 é uma álgebra associativa e comutativa, em 2 é associativa, em 3 é uma álgebra comutativa não associativa. A álgebra em 4 satisfaz as relações $(u \times v) \times w+(v \times w) \times u+(w \times u) \times v=0$ e $u \times v+v \times u=0$, (álgebra de Lie).

Seja A uma álgebra arbitraria, e consideremos as funções comutador e associador em $A$ definidas por:

$$
\begin{aligned}
{[x, y] } & =x y-y x \quad \text { comutador. } \\
(x, y, z) & =(x y) z-x(y z) \text { associador. }
\end{aligned}
$$

para todo $x, y, z, \in A$.

Note que as funções anteriores são lineares em cada componente. Além disso, tem-se que para todo $w, x, y, z$ em $A$ verificam-se as identidades

$$
\begin{array}{r}
w(x, y, z)+(w, x, y) z=(w x, y, z)-(w, x y, z)+(w, x, y z) . \\
(x, y, z)-(x, z, y)+(z, x, y)=[x y, z]-x[y, z]-[x, z] y .
\end{array}
$$

Seja $A$ uma álgebra. Definimos o centro associativo $N_{A}$ de $A$ pelo conjunto $N_{A}=\{z \in A ;(z, x, y)=(x, z, y)=(x, y, z)=0, \quad \forall x, y \in A\}$. Definimos o centro comutativo $Z_{A}$ de $A$ pelo conjunto $Z_{A}=\{c \in A ; \quad c a=a c, \forall a \in A\}$. Finalmente definimos o Centro $C_{A}$ de $A$, pelo conjunto $C_{A}=N_{A} \cap Z_{A}$.

Com as identidades dadas em (1.3) e (1.4), pode-se verificar facilmente que $N_{A}$ é uma subálgebra associativa de $A$ e $C_{A}$ é uma subálgebra associativa e comutativa de $A$. Note também que uma álgebra $A$ é associativa se $N_{A}=A$. 


\section{2 Álgebra de multiplicação}

Seja $a \in A$ e consideremos os operadores $R_{a}, L_{a}$ definidos por

$$
x R_{a}=x a, \quad x L_{a}=a x \quad \forall x \in A .
$$

os quais chamaremos de multiplicações à direita e à esquerda por $a$, respectivamente.

Definem-se as álgebras de multiplicação à direita e à esquerda de $A$ respectivamente por:

$$
R(A)=\operatorname{alg}_{\text {End } A}\left\langle R_{a}, a \in A\right\rangle \quad \text { e } \quad L(A)=\operatorname{alg}_{\text {End } A}\left\langle L_{a}, a \in A\right\rangle
$$

Define-se a álgebra de multiplicação de $A$, ou envolvente de $L(A) \cup R(A)$, a álgebra

$$
M(A)=\operatorname{alg}_{\text {End } A}\left\langle L_{a}, R_{a} \quad a \in A\right\rangle
$$

Assim, os elementos de $M(A)$ são da forma $\sum S_{1} S_{2} \ldots S_{h}$, onde cada $S_{i}$ ou é uma multiplicação à direita ou uma multiplicação à esquerda.

Dado um conjunto $B \subset A$, define-se a álgebra

$$
M^{A}(B)=\operatorname{alg}_{\text {End } A}\left\langle R_{a}, L_{a}: a \in B\right\rangle
$$

Note que para $A \neq B, M^{A}(B) \neq M(B)$.

\section{3 Álgebras simples}

Definição 1.3 Uma álgebra $A$ é dita simples se não contém ideais próprios não nulos e $A^{2} \neq 0$.

Definição 1.4 Uma forma bilinear simétrica $(x, y)$ definida numa álgebra $A$ é chamada uma forma traço (forma associativa) em A se

$$
(x y, z)=(x, y z) \quad \forall x, y, z \in A
$$


Observação 1.5 Sejam A uma álgebra e B um ideal de $A$. Se (, ) é uma forma traço em $A$, então o conjunto $B^{\perp}=\{y \in A /(x, y)=0, \quad \forall x \in B\}$ é um ideal de $A$. Em particular, $A^{\perp}$ é um ideal de $A$, o qual é chamado radical da forma traço de $A$.

Teorema 1.6 (Dieudonné) Seja A uma álgebra de dimensão finita sobre $\mathbb{F}$ tal que, para todo $B \triangleleft A, \quad B^{2} \neq 0$. Se $A$ possui uma forma traço não degenerada

$$
\begin{aligned}
(,): A \times A & \longrightarrow \mathbb{F} \\
a, b & \mapsto(a, b)
\end{aligned}
$$

então A é soma direta de álgebras simples. Isto é,

$$
A=B_{1} \oplus B_{2} \oplus \cdots \oplus B_{n}
$$

onde cada $B_{i}$ é uma álgebra simples. Essa decomposição é única salvo permutações dos $B_{i}$.

Demonstração: Vamos provar por indução na dimensão de $A$. Se $A$ é de climensão 1 e não nula, então $A$ é simples, logo o teorema vale. Suponhamos que o resultado cumpre-se para álgebras de dimensão menor que $n$ e vejamos que também vale para álgebras de dimensão $n$.

Seja $0 \neq B$ um ideal minimal de $A$. Como $($, ) é uma forma traço, então $B^{\perp}$ é um ideal de $A$ e assim, $B \cap B^{\perp} \triangleleft A$. Dada a minimalidade de $B$, tem-se que $B \cap B^{\perp}=0$ ou $B \cap B^{\perp}=B$.

Se $B \cap B^{\perp}=B$, então $B \subset B^{\perp}$, isto é, $(x, y)=0$, para todo $x, y \in B$, portanto $(B, B)=0$. Como $0=(z y, x)=(z, y x)$ para todo $x, y \in B \mathrm{e}$ $z \in A$ então $\left(B^{2}, A\right) \subseteq(B, B A) \subseteq(B, B)=0 \operatorname{assim}\left(B^{2}, A\right)=0$, e $($, ) é não degenerada, $\operatorname{logo} B^{2}=0$, o qual é uma contradição, pois pela hipótese $B^{2} \neq 0$. Podemos logo concluir que $B \cap B^{\perp}=0$, portanto $B$ é não isotropico. Assim, $A=B \perp B^{\perp}$ como soma de espaços vetoriais ortogonais, e como $B^{\perp}$ 
também é não isotropico então $A=B \oplus B^{\perp}$, soma direta de ideais. Logo $\operatorname{dim} B^{\perp}<\operatorname{dim} A=n$ e $\left.()\right|_{,B^{\perp}}$ é uma forma traço não degenerada em $B^{\perp}$, assim pela hipótese indutiva, o teorema vale para $B^{\perp}$, isto é, $B^{\perp}$ é soma de álgebras simples. Finalmente temos que $B$ é simples. Pois se $I$ for ideal de $B$, pela minimalidade de $B, I=0$ ou $I=B$, portanto $B$ é simples. Assim nós concluímos que $A=B_{1} \oplus B_{2} \oplus \cdots \oplus B_{n}$.

Vamos verificar a unicidade da decomposição. Seja.

$$
A=B_{1} \oplus B_{2} \oplus \cdots \oplus B_{n}=C_{1} \oplus C_{2} \oplus \cdots \oplus C_{k}
$$

onde os $B_{i}$ e os $C_{j}$ são simples.

Vejamos que para cada $i$ existe um $j_{i}$ tal que $C_{i}=B_{j_{i}}$, tendo assim $n \leq k$. Temos $C_{i} A=C_{i}{ }^{2}=C_{i} \neq 0$, portanto existe $j_{i}$ tal que $C_{i} B_{j_{i}} \neq 0$. Como $C_{i} B_{j_{i}} \subseteq C_{i} \cap B_{j_{i}}$ então $C_{i} B_{j_{i}} \subset C_{i}$ e $C_{i} B_{j_{i}} \subseteq B_{j_{i}}$ e dada a simplicidade dos $C_{i}, B_{j_{i}}$ temos que $C_{i}=B_{j_{i}}$, assim $k \leq n$. Do mesmo modo se prova $n \leq k$, obtendo assim a igualdade.

Proposição 1.7 Seja $A=A_{1} \oplus A_{2} \oplus \cdots \oplus A_{n}$ uma álgebra, onde cada $A_{i}$ é simples. Então, para todo ideal não nulo I de A tem-se que $I=A_{i_{1}} \oplus \cdots \oplus A_{i_{k}}$.

Demonstração: Seja $I$ ideal não nulo de $A$, logo para cada $b \in I$, te$\operatorname{mos} b=b_{1}+b_{2}+\ldots+b_{n}$ onde não todos os $b_{i}$ são nulos. Seja $M=$ $\left\{m_{1}, m_{2} \ldots, m_{t}\right\} \subset\{1, \ldots, n\}$ um subconjunto não vazio, tal que $b_{m_{j}} \neq 0$ para algum $b \in I$. É evidente, que

$$
I \subseteq \bigoplus_{m \in M} A_{m}
$$

Sejam $m \in M$ e $b \in I$ tal que $b=b_{1}+\cdots+b_{m}+\cdots+b_{n} \operatorname{com} b_{m} \neq 0$. Temos $b A_{m}=b_{m} A_{m} \subseteq A_{m} \cap I$, e $b_{m} A_{m} \neq 0$. Pois se $b_{m} A_{m}=0$ então, $\operatorname{id}_{A_{m}}\left\langle b_{m}\right\rangle A_{m}=0$, e pela simplicidade de $A_{m}$ temos $\operatorname{id}_{A_{m}}\left\langle b_{m}\right\rangle=A_{m}$ portanto $A_{m}^{2}=0$, o que é uma contradição. Assim $0 \neq A_{m} \cap I$ ideal de $A_{m}$ 
portanto $A_{m} \cap I=A_{m}$. Portanto $A_{m} \subseteq I$ para todo $m \in M$. Obtemos assim a igualdade.

Sejam $A, B$ espaços vetoriais sobre $\mathbb{F}$ e consideremos o espaço produto tensorial $A \otimes \mathbb{F} B$, munido com a multiplicação $(a \otimes b)(c \otimes d)=a c \otimes b d$, com $a, c \in A, b, d \in B$. Assim, $A \otimes B$ tem estrutura de álgebra. Esta álgebra é chamada álgebra do produto tensorial. Em particular, para cada. $\mathbb{K} \supset \mathbb{F}$, temos a álgebra extensão de $A, A_{\mathbb{K}}$ considerada como álgebra sobre $\mathbb{K}$, definida por $A_{\mathbb{K}}=\mathbb{K} \otimes_{\mathbb{F}} A$.

Definição 1.8 Uma álgebra A com 1 é chamada central sobre $\mathbb{F}$ se o centro de $A$ coincide com $\mathbb{E}$.

O seguinte teorema vai nos permitir caracterizar as álgebras centrais simples. Vamos considerar isto especialmente no momento em que estudaremos as álgebras de Jordan. Uma demonstração do seguinte resultado encontra-se em [3] ou [2] Teorema 9, página 206.

Teorema 1.9 S'e A é uma álgebra simples e central sobre $\mathbb{F}$ então $A_{\mathbb{K}}$ é simples e central sobre $\mathbb{K}$, para todo corpo $\mathbb{K} \supset \mathbb{F}$.

\section{4 Álgebras livres e variedades de álgebras}

\subsection{1 Álgebras livres}

Seja $V$ um espaço vetorial sobre $\mathbb{F}$. Define-se a álgebra tensorial associativa sobre $V$, por $T(V)=V \oplus(V \otimes V) \oplus(V \otimes V \otimes V) \oplus \ldots$ onde a multiplicação é dada por $v_{i} \cdot v_{j}=v_{i} \otimes v_{j}$.

Sejam

$$
V^{\otimes 1}=V, \quad V^{\otimes 2}=V \otimes V, \quad V^{\otimes n}=\sum_{i=1}^{n}\left(V^{\otimes i}\right) \otimes\left(V^{\otimes n-i}\right) .
$$


consideremos o espaço vetorial $\mathcal{F}\{V\}=\oplus_{i=1}^{n} V^{\otimes i}$ obtido a partir de $V$ e munido da multiplicação $v_{i} \cdot v_{j}=\left(v_{i}\right) \otimes\left(v_{j}\right) \in V^{\otimes i+j}$, onde $v_{i} \in V^{\otimes i}, v_{j} \in V^{\otimes j}$. Assim temos que $\mathcal{F}\{V\}$ possui estrutura de álgebra. Tal álgebra é dita álgebra tensorial não associativa sobre o espaço $V$.

Seja $X=\left\{x_{1}, x_{2}, \ldots\right\}$ uma base de $V$. Então uma base para $T(V)$ é dada pelos produtos associativos e não comutativos nas variáveis $x_{1}, x_{2}, \ldots$, e a álgebra $T(V)$, também denotada por $T(X)$, é dita a álgebra livre associativa nas variáveis livres $x_{1}, x_{2}, \ldots$ Uma base para $\mathcal{F}\{V\}$ é dada pelos produtos não associativos e não comutativos nas variáveis $x_{1}, x_{2}, \ldots$, e a álgebra $\mathcal{F}\{V\}$, também denotada por $\mathcal{F}\{X\}$, é dita a álgebra livre não associativa nas variáveis livres $x_{1}, x_{2}, \ldots$

O seguinte resultado caracteriza uma propriedade universal que nós permite passar do estudo da álgebra livre ao estudo de uma álgebra qualquer.

Proposição 1.10 Seja A uma álgebra (associativa). Todo aplicação $\psi: X \longrightarrow A$ estende-se, de modo único, a um homomorfismo de álgebras $\widetilde{\psi}: \mathcal{F}\{X\} \longrightarrow A(\tilde{\psi}: T(X) \longrightarrow A)$.

\subsubsection{Variedades}

Seja $f=f\left(x_{1}, \ldots, x_{n}\right) \in \mathcal{F}\{X\}$. Dizemos que uma álgebra $A$ satisfaz a. identidade $f=0$ se $f\left(a_{1}, \ldots, a_{n}\right)=0$, para todo $a_{1}, \ldots, a_{n} \in A$. Sejam $L$ um conjunto enúmeravel (finito ou não) e $M=\left\{f_{i}: i \in L\right\}$ um conjunto não vazio da álgebra livre $\mathcal{F}\{X\}$. O conjunto

$V(M)=\left\{A: A\right.$ é uma álgebra que satisfaz a identidade $\left.f_{i}, \quad \forall f_{i} \in M\right\}$ é chamado variedade de álgebras definida por $M$.

Exemplo 1.11 
1. Seja $M=\left\{\left(x_{1} \cdot x_{2}\right) \cdot x_{3}-x_{1} \cdot\left(x_{2} \cdot x_{3}\right)\right\}$. A variedade das álgebras associativas (2(5s). Assim, A é uma álgebra associativa se para todo $a, b, c \in A$ se cumpre:

$$
(a b) c-a(b c)=0 . \quad \text { Associatividade. }
$$

2. Seja $M=\left\{x_{1} \cdot x_{2}-x_{2} \cdot x_{1},\left(\left(x_{1} \cdot x_{1}\right) \cdot x_{2}\right) \cdot x_{1}-\left(x_{1} \cdot x_{1}\right)\left(x_{2} \cdot x_{1}\right)\right\}$. A variedade das álgebras de Jordan, (כ̧ord). Assim, J é uma álgebra de Jordan se para todo $a, b \in J$ se cumpre:

$$
\begin{aligned}
a b-b a & =0 . & & \text { Comutatividade. } \\
\left(a^{2} b\right) a-a^{2}(b a) & =0 . & & \text { Identidade de Jordan. }
\end{aligned}
$$

3. Seja $M=\left\{x_{1} \cdot x_{2}+x_{2} \cdot x_{1},\left(x_{1} \cdot x_{2}\right) \cdot x_{3}+\left(x_{2} \cdot x_{3}\right) \cdot x_{1}+\left(x_{3} \cdot x_{1}\right) \cdot x_{2}\right\}$. A variedade das álgebras de Lie, (¿ie). Assim, L é uma álgebra de Lie se para todo $a, b, c \in L$ se cumpre:

$$
\begin{aligned}
a b+b a & =0 . & & \text { Anticomutatividade } \\
(a b) c+(b c) a+(c a) b & =0 . & & \text { Identidade de Jacobi. }
\end{aligned}
$$

4. Seja $M=\left\{\left(x_{1} \cdot x_{1}\right) \cdot x_{2}-x_{1} \cdot\left(x_{1} \cdot x_{2}\right), x_{2} \cdot\left(x_{1} \cdot x_{1}\right)-\left(x_{2} \cdot x_{1}\right) \cdot x_{1}\right\}$. A variedade das álgebras alternativas, (allt). Assim, A é uma álgebra alternativa se para todo $a, b \in A$ se cumpre:

$$
\begin{array}{ll}
a^{2} b-a(a b)=0 . & \text { Alternatividade à esquerda. } \\
b a^{2}-(b a) a=0 . & \text { Alternatividade à direita. }
\end{array}
$$

Seja $A$ uma álgebra. O conjunto $I(A)=\{f \in \mathcal{F}\{X\}: f=0$ em $A\}$ é dito o conjunto de identidades de $A$. É fácil verificar que $I(A)$ é um ideal de $\mathcal{F}\{X\}$, mais ainda, se $\psi$ é um endomorfismo de $\mathcal{F}\{X\}$ e $f \in I(A)$, então $\psi(f) \in I(A)$, temos assim a seguinte Proposição. 
Proposição 1.12 Seja A uma álgebra. O conjunto de identidades de $A$ é um ideal da álgebra livre $\mathcal{F}\{X\}$ o qual é invariante sob endomorfismos de $\mathcal{F}\{X\}$.

Um ideal $I$ da álgebra associativa livre $\mathcal{F}\{X\}$ o qual é invariante sob endomorfismos de $\mathcal{F}\{X\}$ é dito um $T$-ideal, ou um ideal verbal.

Seja $v$ um monômio em $\mathcal{F}\{X\}$ e seja $x \in X$, define-se o $x$-grau de $v$ por $\operatorname{deg}_{x} v$ como o número de vezes que aparece $x$ na representação de $v$, e define-se o grau de $v$ por $\operatorname{deg} v=\sum_{x_{i}} \operatorname{deg}_{x_{i}} v$. Dado $f=\sum_{i} \alpha_{i} v_{i}$ onde os $v_{i}$ são monômios e $\alpha_{i}$ é não nulo só para um número finito de elementos. Define-se o grau de $f$ em $\mathcal{F}\{X\}$ por $\max _{v_{i}}\left\{\operatorname{deg} v_{i}\right\}$ e define-se o $x$-grau de $f$ por $\max _{v_{i}}\left\{\operatorname{deg}_{x} v_{i}\right\}$.

Definimos $\mathcal{F}^{\#}\{X\}=\mathcal{F}\{X\}+\mathbb{F} \cdot 1$ como a álgebra livre não associativa com unidade, onde $w \cdot 1=1 \cdot w=w$ para qualquer $w \in \mathcal{F}\{X\}$. De modo análogo, dada uma álgebra $A$ podemos definir a álgebra com unidade $A^{\#}=A+\mathbb{F} \cdot 1$.

Proposição 1.13 Seja A uma álgebra de Jordan (alternativa). Então $A^{\#}$ é de Jordan (alternativa).

Demonstração: Vamos considerar o caso das álgebras de Jordan, (para o caso alternativo procede-se de modo análogo). Seja $A$ uma álgebra de Jordan e $a, b \in A$ então, $a b-b a=0$ e $a^{2} b \cdot a-a^{2} \cdot b a=0$ ou en termos das funções comutativa e associativa dadas em (1.1) e (1.2), temos que $[a, b]=\left(a^{2}, b, a\right)=$ 0 . Sim perda de generalidade suponhamos $a^{\#}=a+1, b^{\#}=b+1$, assim $\left[a^{\#}, b^{\#}\right]=[a+1, b+1]=[a, b]=0$ e $\left(\left(a^{\#}\right)^{2}, b^{\#}, a^{\#}\right)=\left((a+1)^{2}, b, a+1\right)=$ $\left(a^{2}+2 a+1, b+1, a+1\right)=\left(a^{2}, b, a\right)+(2 a, b, a)=2(a, b, a)=2(a b \cdot a-a \cdot b a)=$ $2 a \cdot(a b-b a)=0$. 


\subsubsection{Variedades homogêneas}

Um elemento $f\left(x_{1}, \ldots, x_{t}\right)=\sum_{i=1}^{k} \alpha_{i} u_{i}\left(x_{1}, \ldots, x_{t}\right) \in \mathcal{F}\{X\}$ é dito homogêneo de grau $n$ se $\operatorname{deg} u_{i}=\operatorname{deg} f=n$, para todo $i, \operatorname{com} \alpha_{i} \neq 0$. Um elemento $f\left(x_{1}, \ldots, x_{t}\right)=\sum_{i=1}^{k} \alpha_{i} u_{i}\left(x_{1}, \ldots, x_{t}\right) \in \mathcal{F}\{X\}$ é dito multihomogêneo de multi-grau $\left(n_{1}, n_{2}, \ldots, n_{t}\right)$ se para todo $x_{i} \in X$ e para cada monômio $u_{j}$, temos que $\operatorname{deg}_{x_{i}} f=\operatorname{deg}_{x_{i}} u_{j}=n_{i}$.

Uma variedade $\mathfrak{M}$ é dita homogênea se $I(\mathfrak{M})$ é um ideal homogêneo; isto é, para todo $f \in I(\mathfrak{M})$ cada componente homogênea de $f$ pertence a $I(\mathfrak{M})$.

Proposição 1.14 As variedades de álgebras associativas, de Jordan, de Lie e alternativas são variedades homogêneas.

Um elemento $f \in \mathcal{F}\{X\}$ é dito multi-linear se é linear em cada variável.

Definição 1.15 Sejam $M, N$ dois subconjuntos não vazios da álgebra livre $\mathcal{F}\{X\}$. Dizemos que o conjunto de identidades de $M$ é equivalente com o conjunto de identidades de $N$ se, $V(M)=V(N)$.

Proposição 1.16 Sobre um corpo de característica zero, cada identidade é equivalente (no sentido que pode ser substituída) com uma identidade multilinear.

Demonstração: Seja $f=f(x, y, \ldots)$ uma identidade multi-homogênea de multi-grau $(m, n, \ldots)$, assim, $\operatorname{deg}_{x} f=m$ e $\operatorname{deg}_{y} f=n$. Substituindo $x$ por $x_{1}+\lambda x_{2}$ com $\lambda \in \mathbb{F}$ obtemos, $f=f_{0}\left(x_{1}, x_{2} ; y, \ldots\right)+\lambda f_{1}\left(x_{1}, x_{2} ; y, \ldots\right)+$ $\cdots+\lambda^{m} f_{m}\left(x_{1}, x_{2} ; y, \ldots\right)$ onde $\operatorname{deg}_{x_{2}} f_{i}=i$. Em particular $f_{0}\left(x_{1}, x_{2} ; y, \ldots\right)=$ $f\left(x_{1} ; y, \ldots\right)$ e $f_{m}\left(x_{1}, x_{2} ; y, \ldots\right)=f\left(x_{2} ; y, \ldots\right)$ e para $i \neq 0, m$ temos que $\operatorname{deg}_{x_{1}} f_{i}<m$. Assim usando o determinante de Vandermonde obtemos que se $f \in I(\mathfrak{M})$ então cada $f_{i}\left(x_{1}, x_{2} ; y, \ldots\right) \in I(\mathfrak{M})$, e para cada $i \neq 0, m$ $f_{i}\left(x_{1}, x_{2} ; y, \ldots\right)$ tem multi-grau $(m-i, i ; n, \ldots)$ com $m-i, i<m$. Observemos 
ainda que cada $f_{i}$ é equivalente a $f$, pois $f_{i}(x, x ; y, \ldots)=\left(\begin{array}{c}m \\ i\end{array}\right) f(x ; y, \ldots)$. Assim, procedendo por indução no grau das variáveis obtemos que $f$ é equivalente a uma identidade multi-linear.

Observação 1.17 O processo descrito na prova da proposição anterior é chamado de linearização. As identidades obtidas no processo são chamadas de linearizações parciais de $f$.

Das Proposições 1.14 e 1.16, temos que o conjunto de identidades que caracterizam as álgebras de Jordan (Lie, alternativas), são equivalentes a um conjunto de identidades multi-lineares em corpos de característica zero, mais ainda, veremos que no caso das álgebras de Jordan só precisamos que o corpo seja de característica diferente de três.

A seguinte proposição, vai nos permitir estender uma variedade $\mathfrak{M}$, de álgebras $A$ sobre $\mathbb{F}$, a uma variedade $\mathfrak{M}^{\prime}$ de álgebras $A_{\mathbb{K}}$, sendo $\mathbb{K}$ uma extensão associativa, comutativa de $\mathbb{F}$. Note que $\mathbb{K}$ pode ser um anel.

Proposição 1.18 Seja A uma álgebra sobre $\mathbb{F}$ tal que A satisfaz uma identidade multi-linear. Então, para qualquer extensão comutativa, associativa $\mathbb{K}$ de $\mathbb{F}$, a álgebra $A_{\mathbb{K}}=\mathbb{K} \otimes_{\mathbb{F}} A$ também satisfaz a identidade.

Demonstração: $\quad$ Sejam $f\left(x_{1}, \cdots, x_{n}\right)$ uma identidade multi-linear, $a_{1}, \ldots$, $a_{n} \in A$ e $b_{1}, \ldots, b_{n}$ elementos não nulos de $\mathbb{K}$. Quando $f$ é um monômio multi-linear $m\left(x_{1}, \cdots, x_{n}\right)$. Temos pela hipótese, $m\left(a_{1}, \ldots, a_{n}\right)=0$. Consideremos os produtos $b_{i} \otimes a_{i}$ para todo $i=1, \ldots, n$. Temos pela comutatividade e associatividade de $\mathbb{K}$ que $m\left(b_{1} \otimes a_{1}, b_{2} \otimes a_{2}, \ldots, b_{n} \otimes a_{n}\right)=b_{1} b_{2} \cdots b_{n} \otimes$ $m\left(a_{1}, a_{2}, \ldots, a_{n}\right)$ e $m\left(a_{1}, a_{2}, \ldots, a_{n}\right)=0$, portanto, $m\left(b_{1} \otimes a_{1}, \ldots, b_{n} \otimes a_{n}\right)=$ 0 . Temos assim que $m$ é uma identidade em $A_{\mathbb{K}}$. Seja $f=\sum_{i} m_{i}$ e cada $m_{i}$ é um monômio. Então $f\left(b_{1} \otimes a_{1}, b_{2} \otimes a_{2}, \ldots, b_{n} \otimes a_{n}\right)=\sum_{i} m_{i}\left(b_{1} \otimes a_{1}, b_{2} \otimes\right.$ $\left.a_{2}, \ldots, b_{n} \otimes a_{n}\right)=\sum_{i} b_{1} b_{2} \cdots b_{n} \otimes m_{i}\left(a_{1}, \ldots a_{n}\right)=b_{1} \cdots b_{n} \otimes\left(\sum_{i} m_{i}\left(a_{1}, \ldots, a_{n}\right)\right)$ $=b_{1} \cdots b_{n} \otimes f\left(a_{1}, \ldots, a_{n}\right)=\dot{0}$. Portanto, $f$ é identidade em $A_{\mathbb{K}}$. 


\subsection{Radical}

Definição 1.19 (Radical no sentido Amitsur-Kurosh) Seja M uma classe de álgebras a qual é fechada para imagens homomorfas e seja $\Re$ uma subclasse de $\mathfrak{M}$ tal que:

1. R é fechado para imagens homomorfas.

2. Para todo $A$ em $\mathfrak{M}$ existe um ideal $\mathfrak{R}(A)$ de $A$ tal que $\mathfrak{R}(A)$ pertence a $\Re \mathfrak{R}(A)$ contem todos os ideais de $A$ que pertencem a $\Re$.

3. $\mathfrak{R}(A / \Re(A))=0$.

Neste caso, a subclasse $\mathfrak{R}$ é chamada de classe radical em $\mathfrak{M}$ e $\mathfrak{R}(A)$ é chamado de R-radical de $A$.

Se uma álgebra $A$ pertence a $\Re(\Re(A)=A)$, dizemos que ela é uma $\Re$-álgebra, ou que a álgebra $A$ satisfaz a propriedade $\Re$.

Uma álgebra $A \in \mathfrak{M}$ é dita $\mathfrak{R}$-semi-simples se $\mathfrak{R}(A)=0$.

Seja $A$ uma álgebra e $a \in A$. Dizemos que $a$ é nilpotente em $A$ se existe um monômio $v\left(x_{1}, \ldots, x_{n}\right) \in \mathcal{F}\{X\}$ tal que $v(a, \ldots, a)=0$.

Uma álgebra $A$ é dita uma nil-álgebra se todos seus elementos são nilpotentes.

Vamos agora verificar que a classe das nil-álgebras é uma subclasse radical, para isso nós vamos precisar dos seguintes lemas:

Lema 1.20 Se I é um ideal de A tal que I e A/I são nil, então A é nil.

Demonstração: Seja $a \in A$. Como $\bar{a}=a+I \in A / I$ nil, existe um monômio $v(x) \in \mathcal{F}\{X\}$ tal que $\overline{0}=v(\bar{a})=v(a+I)=v(a)+I=\overline{v(a)}$. Assim, temos $v(a) \in I$ e como $I$ é nil, então existe $w(x) \in \mathcal{F}\{X\}$ tal que $w(v(a))=0$. Seja $u(x)=v(v(x)) \in \mathcal{F}\{X\}$, então $u(a)=0$, assim $A$ é nil. 
Lema 1.21 Se $I, J$ são nil-ideais de $A$, então $I+J$ também o é.

Demonstração: Temos que $(I+J) / I \cong J /(I \cap J)$. Portanto, $(I+J) / I$ é nil e, pelo Lema $1.20, I+J$ é nil.

Teorema 1.22 A classe das nil-álgebras é uma subclasse radical na classe de todas as álgebras.

Demonstração: Basta verificar que $A$ possui um nil-ideal maximal. Seja Nil $A=\sum\{I: I$ nil-ideal de $A\}$ e seja $a \in \operatorname{Nil} A$, logo existem $I_{1}, I_{2}, \ldots, I_{n}$ nil-ideais tais que $a \in I_{1}+I_{2}+\cdots+I_{n}$ e, pelo Lema 1.20 , temos que $I_{1}+$ $I_{2}+\cdots+I_{n}$ é nil, portanto $a$ é nilpotente e Nil $A$ é nil. Seja $I$ um nil-ideal de $A, \operatorname{logo} I \subset \mathrm{Nil} A$. Assim temos que $\mathrm{Nil} A$ é ideal maximal.

Vejamos agora que $\operatorname{Nil}(A / \mathrm{Nil} A)=0$. Seja $\bar{I} \subset A / \mathrm{Nil} A$ um nil-ideal. Pelo Teorema da correspondência existe um ideal $I$ de $A$ tal que Nil $A \subset I$ e $I / \mathrm{Nil} A \cong \bar{I}$, mas $I / \mathrm{Nil} A$ é nil, e pelo Lema $1.20, I$ é nil, portanto $I \subset \operatorname{Nil} A$, e $\bar{I}=\overline{0}$.

O ideal Nil $A$ é chamado de nil radical de $A$.

Dada uma álgebra $A$ define-se

$$
A^{(0)}=A, A^{(1)}=A^{2}, A^{(k+1)}=\left(A^{(k)}\right)^{2}
$$

e

$$
A^{1}=A, A^{2}=A A, A^{n}=\sum_{\substack{i+j=n \\ i, j>0}} A^{i} A^{j} .
$$

Dizemos que $A$ é solúvel se existe um inteiro $n$ tal que $A^{(n)}=0$. Dizemos que $A$ é nilpotente se existe um inteiro $m$ tal que $A^{m}=0$.

Teorema 1.23 A classe das álgebras solúveis é uma subclasse radical na classe de todas as álgebras de dimensão finita. 
A demonstração deste resultado é análoga à feita no Teorema 1.22.

Consideremos o seguinte.

1. Se $A$ é uma álgebra e $I$ é um ideal de $A$ tais que $I$ e $A / I$ são solúveis, então $A$ também é solúvel.

2. Se $I, J$ são ideais de $A$ não nulos, tais que tanto $I$ como $J$ são solúveis, então $I+J$ também o é.

3. $\operatorname{Solv}(A)=\left\{\sum I, I\right.$ ideal solúvel de $\left.A\right\}$.

Observação $1.24 \mathrm{Na}$ classe das álgebras não associativas de dimensão finita, a subclasse das álgebras nilpotentes (Nilp) não é uma classe radical, como mostra o seguinte exemplo.

Exemplo 1.25 Considere a álgebra $A=\mathbb{F} a+\mathbb{F} b+\mathbb{F} e_{1}+\mathbb{F} e_{2}$, com multiplicação não nula dada por $e_{1} \cdot a=a \cdot e_{1}=e_{2}, \quad e_{2} \cdot b=b \cdot e_{2}=e_{1}$. Assim, A possui dois ideais nilpotentes maximais $I_{1}=\mathbb{F} a+\mathbb{F} e_{1}+\mathbb{F} e_{2}, \quad I_{2}=$ $\mathbb{F} b+\mathbb{F} e_{1}+\mathbb{F} e_{2}$.

Do mesmo modo que foram definidas $A^{(n)}$ e $A^{n}$, podemos definir para um elemento $a \in A, a^{1}=a, a^{2}=a a, a^{n}=a^{n-1} a$ e $a^{(0)}=a, a^{(1)}=a^{2}, a^{(n+1)}=$ $\left(a^{(n)}\right)^{2}$.

Proposição 1.26 Toda álgebra A de dimensão finita e solúvel é nil.

Demonstração: Seja $a \in A$. Se $A$ é solúvel de índice $n$, então $A^{(n)}=0$, logo para todo $a \in A$ tem-se $a^{(n)}=0$, portanto $A$ é nil.

Seja $\mathfrak{M}$ a subclasse da classe das álgebras de dimensão finita, e suponhamos que a subclasse $\mathrm{Nilp}$ de álgebras nilpotentes é uma classe radical, então tem-se que para todo $A \in \mathfrak{M}$ o radical nilpotente está contido no radical 
solúvel, e o radical solúvel, pelo teorema anterior, está contido no radical nil, isto é:

$$
\text { Nilp } A \subseteq \operatorname{Solv} A \subseteq \operatorname{Nil} A
$$

Uma álgebra $A$ é dita de potências associativas (p.a.) se para todo $a \in$ $A$ e para todo monômio multilinear $v\left(x_{1}, \ldots, x_{n}\right) \in \mathcal{F}\{X\}$ verifica-se que $v(a, \ldots, a)=a^{n}$, isto é, a álgebra gerada por $a,(\operatorname{alg}\langle a\rangle)$ é associativa.

Observação 1.27 Seja $A$ uma álgebra de potências associativas. Como as potências estão definidas univocamente, pode-se definir o índice de nilpotência para qualquer elemento nilpotente $a \in A$, onde $a$ é nilpotente de índice $n$, se e somente se, $a^{n}=0$, ou seja, qualquer produto de $n$ fatores iguais a $a$ é 0 .

Seja $A$ uma álgebra. Dizemos que um elemento $e$ em $A$ é idempotente, se satisfaz $e^{2}=e \neq 0$. Dizemos que dois idempotentes $e, f$ são ortogonais se $e f=f e=0$. Neste caso $e+f$ é idempotente. Um idempotente $e$ é chamado primitivo, se não existem idempotentes ortogonais $f, g$ tais que $e=f+g$.

Proposição 1.28 Seja A uma álgebra p.a. de dimensão finita e não nil. Então A contém um elemento idempotente e.

Demonstração: Ver Proposição 3.3 de [1], página 32.

\subsection{Bimódulos}

Um espaço vetorial $M$ é um $A$-bimódulo para uma álgebra $A$ se existem aplicações bilineares $A \times M \longrightarrow M,(a, m) \mapsto a \cdot m$ e $M \times A \longrightarrow M$, $(m, a) \mapsto m \cdot a$.

Sejam $A$ uma álgebra e $M$ um $A$-bimódulo. Seja $E(A, M)=A+M$ onde $\dot{+}$ representa a soma direta de espaços vetoriais, e consideremos a multiplicação $(a+m)(b+n)=a b+(a \cdot n+m \cdot b)$. Temos que $A$ é uma subálgebra 
de $E(A, M)$ e $M$ é um ideal, com $M^{2}=0$. A álgebra $E(A, M)$ é chamada extensão split de $A$ por $M$.

Seja $\mathfrak{M}$ uma variedade de álgebras e seja $A \in \mathfrak{M}$. Um $A$-bimódulo $M$ é dito um $\mathfrak{M}$-bimódulo se $E(A, M) \in \mathfrak{M}$.

Exemplo 1.29 No caso das álgebras de Jordan, temos que $M$ é um J-bimódulo, (bimódulo de Jordan) se e somente se,

$$
\begin{array}{r}
\left(a^{2}, m, a\right)=0, \\
a \cdot m=m \cdot a, \\
\left(a^{2}, b, m\right)+2(a \cdot m, b, a)=0
\end{array}
$$

Seja $A$ uma álgebra, $M$ um $A$-bimódulo e consideremos as aplicações $\rho, \lambda$ : $A \longrightarrow$ End $M$ tais que para todo $a \in A, \rho(a)=\rho_{a}: M \longrightarrow M$ e para todo $m \in M$ temos $\rho_{a}(m)=m \cdot a$. Definimos $\lambda(a)=\lambda_{a}: M \longrightarrow M$ tal que para todo $m \in M, \lambda_{a}(m)=a \cdot m$ para todo $a \in A$. Seja $(\rho, \lambda)(A)$ a subálgebra de End $M$ gerada por $\rho(A) \cup \lambda(A)$. Assim, temos que $M$ é um módulo à esquerda associativo de $(\rho, \lambda)(A)$.

Para toda álgebra $A \in \mathfrak{M}$ existe álgebra universal associativa $U_{\mathfrak{M}}(A), \mathrm{e}$ aplicações $\mathcal{R}, \mathcal{L}: A \longrightarrow U_{\mathfrak{M}}(A)$ tais que para cada $A$-bimódulo $M$ existe um único homomorfismo de álgebras $\phi: U_{\mathfrak{M}}(A) \longrightarrow$ End $M$ tal que os diagramas
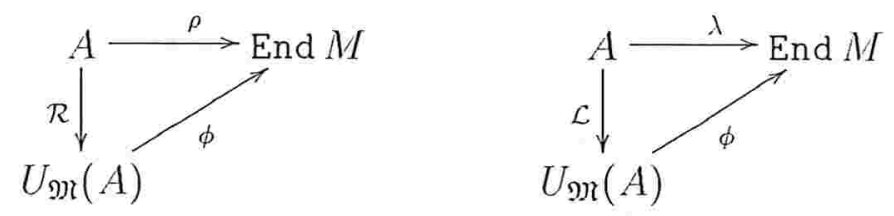

comutam.

A álgebra $U_{\mathfrak{M}}(A)$ é chamada $\mathfrak{M}$-envolvente multiplicativa de $A$. 


\section{Capítulo 2}

\section{ÁLGEBRAS DE JORDAN DE DIMENSÃO FINITA}

Uma álgebra de Jordan $J$ é uma álgebra sobre um corpo $\mathbb{F}$ com característica diferente de 2 tal que $J$ é comutativa e verifica a relação

$$
\left(a^{2}, b, a\right)=0 . \quad \forall a, b \in J
$$

A identidade (2.1) é chamada identidade de Jordan.

Mediante o processo de linearização descrito na Proposição 1.16, temos que as seguintes identidades também irão caracterizar as álgebras de Jordan.

$$
\begin{array}{r}
2(a c, b, a)+\left(a^{2}, b, c\right)=0 \quad \forall a, b, c \in J \\
(a c, b, d)+(d c, b, a)+(a d, b, c)=0 \quad \forall a, b, c, d \in J
\end{array}
$$

A Identidade em (2.2) é chamada linearização parcial, e a Identidade em (2.3) é chamada linearização completa da Identidade (2.1). Em corpos de característica diferente de 3 a Identidade (2.1) é equivalente à Identidade (2.3). 


\section{$2.1 \quad$ Álgebras de Jordan especiais}

Seja $A$ uma álgebra associativa sobre $\mathbb{F}$ com característica diferente de 2 . Vamos considerar $A^{+}$uma álgebra com os elementos de $A$, mas com multiplicação dada pelo produto simétrico $a \cdot b=\frac{1}{2}(a b+b a)$, onde $a b$ representa a multiplicação em $A$.

Observação 2.1 As identidades a seguir são fornecidas pelo produto $\cdot$

1. $a \cdot a=a^{2}=a^{2}$, podemos assim garantir que $a^{\cdot k}=a^{k}$. Isto é, o produto ponto preserva potências.

2. $a \cdot b=b \cdot a$

3. $\left(a^{2} \cdot b\right) \cdot a=a^{2} \cdot(b \cdot a)$

4. $2(b \cdot a) \cdot a-b \cdot a^{2}=a b a$

5. $a^{3} \cdot b+2((a \cdot b) \cdot a) \cdot a=3\left(a^{2} \cdot b\right) \cdot a$

6. $a^{2} \cdot b^{2}-\left(a \cdot b^{2}\right) \cdot a=2((a \cdot b) \cdot a) \cdot b-2(a \cdot b)^{2}$

7. Linearizando 4 temos que

$$
a b c+c b a=2 a \cdot b \cdot c+2 c \cdot b \cdot a-2 a \cdot c \cdot b .
$$

onde $a \cdot b \cdot c:=(a \cdot b) \cdot c$.

Das propriedades 2 e 3 na Observação 2.1 temos que $A^{+}$é uma álgebra de Jordan.

Definição 2.2 Uma álgebra de Jordan $J$ é dita especial se existe uma álgebra associativa A tal que $J$ pode ser imersa na álgebra $A^{+}$. Se uma álgebra de Jordan $J$ não é especial, então dizemos que $J$ é uma álgebra de Jordan excepcional. 
Teorema 2.3 (Macdonald) Seja J uma álgebra de Jordan e $f(x, y, z)$ uma identidade tal que o grau de $f$ em alguma variável é no máximo 1. Se $f$ é uma identidade em toda álgebra especial de Jordan, então também o é em qualquer álgebra de Jordan.

Teorema 2.4 (Shirshov) A álgebra de Jordan livre $F J^{2}$ com dois geradores e 1 é especial.

Teorema 2.5 (Shirshov-Cohn) Qualquer álgebra de Jordan gerada por dois elementos é especial.

A demonstração dos Teoremas 2.3, 2.4 e 2.5 são muito técnicas e não são apresentadas no presente material, para uma prova delas, consultar [2], [3].

Teorema 2.6 (Herstein) Se A é uma álgebra associativa simples, então $A^{+}$é simples.

Demonstração: Sejam $a, b, c \in J$. Definimos o triplo produto de Jordan pela seguinte aplicação linear:

$$
\{a b c\}=2 a \cdot b \cdot c+2 c \cdot b \cdot a-2 a \cdot c \cdot b .
$$

Onde o produto $a \cdot b \cdot c$ está representado a associatividade $(a \cdot b) \cdot c$.

Seja $I$ um ideal de $A^{+}, I \neq 0$. Logo, para todo $a \in A, \quad i \in I$ temos que $i \cdot a \in I$. De (2.5) temos que $\{i a b\} \in I$ para todo $a, b \in A$ e para todo $i \in I$, em particular

$$
2 i a i=\{i a i\}=2 i \cdot a \cdot i+2 i \cdot a \cdot i-a \cdot i^{2} \in I
$$

donde $i^{2} \cdot a=4 i \cdot a \cdot i-2 i a i$, assim, $\operatorname{id}_{\mathrm{A}}\left\langle i^{2}\right\rangle \subseteq I$ para todo $i \in I$.

Pela simplicidade de $A$, temos os seguintes dois casos:

1. Existe um $i \in I$ tal que $i^{2} \neq 0$, neste caso, $A=i d_{\mathbf{A}}\left\langle i^{2}\right\rangle \subseteq I$ assim $I=A$. 
2. Se $\operatorname{id}_{\mathbb{A}}\left\langle i^{2}\right\rangle=0 \forall i \in I$, então para todo $a \in A$, pela Identidade (2.6), temos que $i a i \equiv 0 \bmod \left\langle i^{2}\right\rangle . \quad$ Logo $i A i=0$. Seja $I_{1}=i d_{A}\langle i\rangle$. Se $I_{1} \neq 0$, então $I_{1}=A$, mas $I_{1}{ }^{2}=0$. Portanto, $A$ não é simples, o que é uma contradição.

\subsubsection{Exemplos de álgebras de Jordan}

Definição 2.7 Seja A uma álgebra e * uma aplicação linear em A. Dizemos que * é uma involução em A se $\left(a^{*}\right)^{*}=a$ e $(a b)^{*}=b^{*} a^{*}$.

Seja $A$ uma álgebra e * uma involução em $A$. Dizemos que um elemento $a$ em $A$ é *-simétrico se $a^{*}=a$.

1. Álgebras do tipo Hermitiano $H(A, *)$ :

Seja $A$ uma álgebra associativa com involução * e $H(A, *)$ o conjunto dos elementos *-simétricos de $A$. $H(A, *)$ é uma subálgebra de $A^{+}$. Se $A$ for *-simples, isto é, $A$ não contém ideais próprios invariantes por *, então $H(A, *)$ é simples.

2. Álgebras do tipo Clifford $J(V, f)$ :

Seja $V$ um espaço vetorial sobre um corpo $\mathbb{F}$ e $f$ uma forma bilinear simétrica em $V$. Definimos $J(V, f)=V+\mathbb{F} \cdot 1$, com multiplicação

$$
(v+\alpha \cdot 1)(w+\beta \cdot 1)=\alpha w+\beta v+(f(v, w)+\alpha \beta) \cdot 1
$$

$J(V, f)$ é uma subálgebra da álgebra $C l(V, f)^{+}$induzida pela álgebra de Clifford $C l(V, f)$. Se $f$ é não degenerada e $\operatorname{dim} V>1$, então $J(V, f)$ é simples.

3. Álgebra do tipo Albert $H\left(\mathrm{O}_{3}\right)$ : 
Seja $\mathcal{O}$ a álgebra de octônios (ou números de Cayley), com base $\left\{1, e_{1}, e_{2}, \ldots, e_{7}\right\}$ e com multiplicação não nula dada pela seguinte tabela

\begin{tabular}{r|r|r|r|r|r|r|r|r|}
$\cdot$ & 1 & $e_{1}$ & $e_{2}$ & $e_{3}$ & $e_{4}$ & $e_{5}$ & $e_{6}$ & $e_{7}$ \\
\hline 1 & 1 & $e_{1}$ & $e_{2}$ & $e_{3}$ & $e_{4}$ & $e_{5}$ & $e_{6}$ & $e_{7}$ \\
\hline$e_{1}$ & $e_{1}$ & -1 & $e_{4}$ & $e_{7}$ & $-e_{2}$ & $e_{6}$ & $-e_{5}$ & $-e_{3}$ \\
\hline$e_{2}$ & $e_{2}$ & $-e_{4}$ & -1 & $e_{5}$ & $e_{1}$ & $-e_{3}$ & $e_{7}$ & $-e_{6}$ \\
\hline$e_{3}$ & $e_{3}$ & $-e_{7}$ & $-e_{5}$ & -1 & $e_{6}$ & $e_{2}$ & $-e_{4}$ & $e_{1}$ \\
\hline$e_{4}$ & $e_{4}$ & $e_{2}$ & $-e_{1}$ & $-e_{6}$ & -1 & $e_{7}$ & $e_{3}$ & $-e_{5}$ \\
\hline$e_{5}$ & $e_{5}$ & $-e_{6}$ & $e_{3}$ & $-e_{2}$ & $-e_{7}$ & -1 & $e_{1}$ & $e_{4}$ \\
\hline$e_{6}$ & $e_{6}$ & $e_{5}$ & $-e_{7}$ & $e_{4}$ & $-e_{3}$ & $-e_{1}$ & -1 & $e_{2}$ \\
\hline$e_{7}$ & $e_{7}$ & $e_{3}$ & $e_{6}$ & $-e_{1}$ & $e_{5}$ & $-e_{4}$ & $-e_{2}$ & -1
\end{tabular}

Seja a involução $a \mapsto \bar{a}$ em $\mathcal{O}$ definida por

$$
a=\alpha_{0}+\sum_{i=1}^{7} \alpha_{i} e_{i} \longmapsto \bar{a}=\alpha_{0}-\sum_{i=1}^{7} \alpha_{i} e_{i} .
$$

Seja $H\left(M_{3}(\mathcal{O})\right.$, *) o conjunto de matrizes hermitianas $3 \times 3$ com entradas em $\mathcal{O}$ e involução standard indusida pela involução em $\mathcal{O}$. Então $H\left(M_{3}(\mathcal{O}), *\right)$ é uma álgebra de Jordan.

Uma álgebra de Jordan $J$ é dita do tipo Albert se $K \otimes_{\mathbb{F}} J \cong H\left(M_{3}(\mathcal{O}), *\right)$ para alguma extensão $K$ de $\mathbb{F}$. Denotamos $H\left(\mathcal{O}_{3}\right):=H\left(M_{3}(\mathcal{O}), *\right)$.

As álgebras em (1), (2) são especiais e (3) é excepcional.

O seguinte teorema demonstrado por Zelmanov presenta uma classificação de todas as álgebras de Jordan simples ainda em dimensão infinita.

Teorema 2.8 [Zelmanov] Toda álgebra de Jordan simples é de algum dos tipos $H(A, *), J(V, f), H\left(\mathcal{O}_{3}\right)$. 


\subsection{Envolventes multiplicativas}

Seja $J$ uma álgebra de Jordan. Como vimos no Capítulo 1, a álgebra de multiplicação de J é definida como subálgebra dos endomorfismos de $J$, $M(J)=\operatorname{alg}_{\text {End } J}\left\langle R_{a}, L_{a} \quad a \in J\right\rangle$. Dada a comutatividade em $J$, temos que $R(J)=L(J)=M(J)$ (a álgebra de multiplicação de $J$ é igual à álgebra de multiplicações à direita de $J$ ). Além disso, como já foi observado, se $J \subseteq A$ e $J \neq A$ então, $R^{A}(J)=\operatorname{alg}_{\text {End } A}\left\langle R_{a}, a \in J\right\rangle \neq R(J)=\operatorname{alg}_{\text {End } J}\left\langle R_{a}, a \in J\right\rangle$, mas existe um epimorfismo $\Phi: R^{A}(J) \rightarrow R(J)$ para qualquer álgebra $A$ que contém $J$, onde $R_{a}^{A} \mapsto R_{a}=\left.R_{a}^{A}\right|_{J}$. Neste sentido, existe uma álgebra universal $\mathcal{R}(J)$ com aplicação $a \mapsto \mathcal{R}_{a}$ tal que para toda álgebra $A$ que contém $J$, existe um epimorfismo que faz o diagrama abaixo comutar

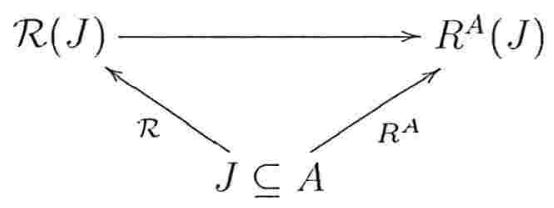

Uma construção para $\mathcal{R}(J)$ é como segue:

Sejam $T(J)=J \oplus(J \otimes J) \oplus(J \otimes J \otimes J) \oplus \ldots$, a álgebra tensorial associativa sobre o espaço $J$ e $\mathcal{R}(J)=T(J) / I$, onde

$I=i \mathrm{~d}_{T(J)}\left\langle a^{2} \otimes a-a \otimes a^{2}, 2 a \otimes b \otimes a+a^{2} b-2 a b \otimes a-a^{2} \otimes b, \quad a, b \in J\right\rangle$

Consideremos a aplicação $\mathcal{R}: J \longrightarrow \mathcal{R}(J)$ onde $a \mapsto \mathcal{R}_{a}=a+I$.

Pela definição do ideal $I$, temos na álgebra $\mathcal{R}(J)$ as seguintes relaçoes:

$$
\begin{gathered}
\mathcal{R}_{a^{2}} \mathcal{R}_{a}=\mathcal{R}_{a} \mathcal{R}_{a^{2}} \\
2 \mathcal{R}_{a} \mathcal{R}_{b} \mathcal{R}_{a}+\mathcal{R}_{a^{2} b}=2 \mathcal{R}_{a b} \mathcal{R}_{a}+\mathcal{R}_{a^{2}} \mathcal{R}_{b}
\end{gathered}
$$

Observamos que casos particulares das relações (2.7), (2.8), podem ser obtidas das identidades (2.1), (2.2), e (2.3).

A álgebra $\mathcal{R}(J)$ é chamada envolvente universal multiplicativa de $J . \mathcal{R}(J)$ é isomorfa com a álgebra $U_{\text {Joro }}(J)$ descrita na Seçã̃o 1.6. 


\subsubsection{Identidades em $\mathcal{R}(J)$}

Daqui em diante vamos escrever $R_{a}$ em lugar de $\mathcal{R}_{a}$.

Linearizando as Relações dadas em (2.7) e (2.8), temos que as seguintes identidades em $\mathcal{R}(J)$.

$$
\begin{gathered}
R_{a^{2} b}+2 R_{a} R_{b} R_{a}=R_{b} R_{a^{2}}+2 R_{a} R_{a b}=R_{a^{2}} R_{b}+2 R_{a b} R_{a} \\
R_{a c \cdot b}+R_{a} R_{b} R_{c}+R_{c} R_{b} R_{a}=R_{a b} R_{c}+R_{b c} R_{a}+R_{a c} R_{b}
\end{gathered}
$$

Dada a simetria do lado direito em $a, b, c$ na Identidade (2.10), temos:

$$
R_{a c \cdot b}+R_{a} R_{b} R_{c}+R_{c} R_{b} R_{a}=R_{a \cdot c b}+R_{b} R_{a} R_{c}+R_{c} R_{a} R_{b}
$$

Portanto

$$
\begin{aligned}
R_{a c \cdot b-a \cdot c b} & =-\left(R_{a} R_{b}-R_{b} R_{a}\right) R_{c}+R_{c}\left(R_{b} R_{a}-R_{a} R_{b}\right) \\
& =-\left[R_{a}, R_{b}\right] R_{c}+R_{c}\left[R_{b}, R_{a}\right] \\
& =-\left[\left[R_{a}, R_{b}\right], R_{c}\right]
\end{aligned}
$$

donde

$$
R_{(a, c, b)}=\left[\left[R_{b}, R_{a}\right], R_{c}\right]
$$

Proposição 2.9 Seja $J=\operatorname{alg}\left\langle a_{\alpha}, \alpha \in I\right\rangle$ uma álgebra de Jordan, então $\mathcal{R}(J)=\operatorname{alg}\left\langle R_{a_{\alpha}}, R_{a_{\alpha} a_{\beta}}: \alpha, \beta \in I\right\rangle$. Em particular, se $J$ é finitamente gerada, então $\mathcal{R}(J)$ também o é.

Demonstração: Da Identidade (2.10), tem-se

$$
R_{a b \cdot c}=-R_{a} R_{c} R_{b}-R_{b} R_{c} R_{a}+R_{a b} R_{c}+R_{a c} R_{b}+R_{b c} R_{a} .
$$

Sabemos que $\tilde{\mathcal{R}}(J)=\operatorname{alg}\left\langle R_{a_{\alpha}}, R_{a_{\alpha} a_{\beta}}: \alpha, \beta \in I\right\rangle \subseteq \mathcal{R}(J)$. 
Provemos a inclusão contrária. Para isso, basta provar que para qualquer produto $u$ de geradores de $a_{\alpha}$ temos que $R_{u} \in \tilde{\mathcal{R}}(J)$. Fazendo indução sobre o número de fatores em $u$ temos: se $u$ tem 1 ou 2 fatores, então, pela definição de $\tilde{\mathcal{R}}(J)$, temos $R_{u} \in \tilde{\mathcal{R}}(J)$. Para $u$ com 3 ou mais fatores, vale a Identidade (2.12).

Corolário 2.10 Se $J=\operatorname{alg}\langle a\rangle$, então $\mathcal{R}(J)=\operatorname{alg}\left\langle R_{a}, R_{a^{2}}\right\rangle$ é comutativa.

Demonstração: Segue-se de $\left[R_{a^{2}}, R_{a}\right]=0$.

Corolário 2.11 Toda álgebra de Jordan é de potências associativas.

Demonstração: Só precisamos verificar que para elementos arbitrários $a^{k}, a^{l}, a^{m}$ cumpre-se $\left(a^{k}, a^{l}, a^{m}\right)=0$. Mas nós temos que

$$
\left(a^{k}, a^{l}, a^{m}\right)=a^{l}\left(R_{a^{k}} R_{a^{m}}-R_{a^{m}} R_{a^{k}}\right)=0
$$

pois $R_{a^{k}}, R_{a^{m}} \in \operatorname{alg}\left\langle R_{a}, R_{a^{2}}\right\rangle$, que, pelo Corolário anterior, é comutativa.

\section{3 Álgebras de Jordan de dimensão finita}

Na presente seção pretendemos verificar que no caso das álgebras de Jordan $J$ de dimensão finita o nil-radical $\mathrm{Nil} J$ é nilpotente, com o qual vamos ter em $J$ que o nil-radical de $J$ e o radical solúvel de $J$ coincidem e são nilpotentes, neste caso valendo a igualdade

$$
\text { Nilp } J=\operatorname{Solv} J=\text { Nil } J
$$

Pela Observação dada em 1.27, como toda álgebra de Jordan é de p.a. então em particular $a$ é nilpotente em $J$ de índice de nilpotência $n$, se e somente se $a^{n}=0$. 
Lema 2.12 Seja $J=B+F \cdot v$ uma álgebra de Jordan tal que $J^{2} \subseteq B$. Se $\mathcal{R}(B)$ é nilpotente, então $\mathcal{R}(J)$ também o é.

Demonstração: $\quad$ Seja $\mathcal{R}^{J}(B)=\operatorname{alg}_{\mathcal{R}(J)}\left\langle\mathcal{R}_{b} / b \in B\right\rangle$. Evidentemente, $\mathcal{R}^{J}(B)$ é imagem homomorfa de $\mathcal{R}(B)$ e portanto é nilpotente também.

Como $J^{2} \subseteq B$, então, para todo $a, b$ em $J$ temos que $a b \in B$. Portanto $R_{a b} \in \mathcal{R}^{J}(B)$.

$$
\begin{gathered}
\text { Seja } S_{a, b, c}=R_{a} R_{c b}+R_{b} R_{c a}+R_{c} R_{a b}-R_{b \cdot c a} \quad \forall a, b, c \in J . \text { Logo } \\
S_{a, b, c} \in \mathcal{R}(J) \mathcal{R}^{J}(B)+\mathcal{R}^{J}(B) \quad \forall a, b, c \in J .
\end{gathered}
$$

Afirmação 1: $\mathcal{R}(J)^{3} \subseteq \mathcal{R}^{J}(B)+\mathcal{R}(J) \mathcal{R}^{J}(B)$.

Denotamos $T^{*}(J)=\mathcal{R}^{J}(B)+\mathcal{R}(J) \mathcal{R}^{J}(B)$. Temos que o produto de três multiplicações à direita pode-ser escrito como uma combinação linear de produtos da forma $R_{a} R_{b} R_{c}$ para $a, b, c \in B \cup\{v\}$. Vamos demonstrar, então, que todo produto $R_{a} R_{b} R_{c}$ pertence a $T^{*}(J)$. Para isso, vamos considerar os seguintes casos:

i) Se $a \in B$ então $R_{a} \in \mathcal{R}^{J}(B)$. Logo a inclusão vale.

ii) Temos da identidade 2.10 que

$$
R_{a} R_{b} R_{c}=S_{a, b, c}-R_{c} R_{b} R_{a}
$$

Logo, tomando $a=v$, e $b, c \in B \cup\{v\}$, temos dois casos:

ii.i) Se $c \in B$ então $R_{c} R_{b} R_{v} \in T^{*}(J)$. Portanto, $R_{v} R_{b} R_{c}=S_{v, b, c}-R_{c} R_{b} R_{v}$ pertence a $T^{*}(J)$.

ii.ii) Se $c=v$, temos

$$
2 R_{v} R_{b} R_{v}=S_{v, b, v}^{\prime} \in T^{*}(J)
$$

De qualquer forma, temos que $R_{a} R_{b} R_{c} \in T^{*}(J)$ e assim a afirmação vale. 
Afirmação 2: $\mathcal{R}(J)^{3 m} \subseteq\left[\mathcal{R}^{J}(B)\right]^{m}+\mathcal{R}(J)\left[\mathcal{R}^{J}(B)\right]^{m}$. Fazendo indução em $m$, temos que, para $m=1$, vale a afirmação 1 .

$$
\mathcal{R}(J)^{3(m+1)} \subseteq\left[\mathcal{R}^{J}(B)\right]^{3 m} \mathcal{R}(J) \subseteq\left(\mathcal{R}^{J}(B)\right)^{m+1}+\left(\mathcal{R}^{J}(B)\right)^{m+1} \mathcal{R}(J) .
$$

Dada a nilpotência de $\mathcal{R}^{J}(B)$ e a afirmação 2 , temos que $\mathcal{R}(J)$ é nilpotente.

Lema 2.13 Seja $B \subseteq J$ uma subálgebra, $a \in J$ tal que $a B \subseteq B$. Então:

1. $\left(a^{2} B\right) B \subseteq B$.

2. $\left(a^{2} B\right)^{2} \subseteq B$.

\section{Demonstração:}

1. Sejam $b, b_{1} \in B$. Temos em termos do associador, que $\left(a^{2} b\right) b_{1}=$ $a(a b) b_{1}+(a, a, b) b_{1}$, e por (2.11), $R_{(a, a, b)}=\left[\left[R_{b}, R_{a}\right] R_{a}\right]$, mas $B R_{b} \subset B, B R_{a} \subset$ $B \operatorname{logo}(a, a, b) b_{1} \in B$ e $a(a b) b_{1} \in B$. Temos assim que $\left(a^{2} b\right) b_{1} \in B$.

2. Note-se que $\left(a^{2} b\right)\left(a^{2} b_{1}\right)=-2\left(\left(a \cdot a^{2} b_{1}\right) b\right) a+\left(\left(a^{2} b_{1} \cdot a\right) a\right) b+\left(\left(a^{2} b_{1}\right.\right.$. b) $a) a+(a b \cdot a) \cdot a^{2} b_{1}$, e como $\left(\left(a \cdot a^{2} b_{1}\right) b\right) a \in B$ e $\left(\left(a^{2} b_{1} \cdot a\right) a\right) b=\left(\left(a b_{1} \cdot a^{2}\right) a\right) b=$ $\left(\left(b_{2} a^{2}\right) a\right) b=\left(b_{2} a \cdot a^{2}\right) b \in B$, então, $\left(a^{2} b\right)\left(a^{2} b_{1}\right) \in B$.

Teorema 2.14 Seja J uma álgebra de Jordan nil e de dimensão finita. Então $\mathcal{R}(J)$ é nilpotente.

Demonstração: Consideremos em $J$ a subálgebra $B$ com a propriedade de que $\mathcal{R}(B)$ é nilpotente (tal subálgebra sempre existe, $B=0$ ). Como $J$ tem dimensão finita entonces existe $B$ com propriedade de dimensão maxima. Como $\mathcal{R}(B)$ é nilpotente entonces $\mathcal{R}^{J}(B)$ tambem é nilpotente, logo existe um inteiro $m$ tal que $\left(R^{J}(B)\right)^{m}=0$, portanto $\left.(\cdots(J B) B) \cdots\right) B$ é zero. 
Seja $n$ o menor inteiro tal que

$$
\underbrace{(\cdots(J B) B) \cdots B)}_{n} \subseteq B
$$

$\mathrm{e}$

$$
\underbrace{(\cdots(J B) B) \cdots B)}_{n-1} \nsubseteq \nsubseteq B
$$

portanto existe $a \in J$ tal que $a \notin B$, e $a B \subset B$. Temos então dois casos:

1. $a^{2} B \subseteq B$ donde $B R_{a^{2}} \subset B$. Como $a B \subset B$, então $B R_{a} \subset B$. Além disso, $R_{a^{k}} \subseteq$ alg $\left\langle R_{a^{2}}, R_{a}\right\rangle$, para todo $k$. Segue que $B R_{a^{k}} \subseteq B, \forall k$. Assim $a^{k} B \subseteq B, \forall k$. Seja $k$ tal que $a^{k-1} \notin B$ e $a^{k} \in B$. Denotemos esse elemento por $s:=a^{k-1}$. $s \notin B$ mas $s^{2} \in B$, com $s B \subseteq B$. Seja $B_{1}=B+F \cdot s$. Temos que $B \triangleleft B_{1}$ e $B_{1}{ }^{2} \subseteq B$. Logo, pelo Lema 2.12, $\mathcal{R}\left(B_{1}\right)$ é nilpotente, o que é uma contradição, pois $B$ é maximal com essa propriedade.

2. Existe $b \in B$ tal que $a^{2} b \notin B$. Seja $a_{1}=a^{2} b$. Como $a B \subseteq B$, segue-se do Lema 2.13, $(a B)^{2} \subseteq B$. Logo $a_{1}{ }^{2} \in B$ e $\left(a^{2} B\right) B \subseteq B$. Portanto $a_{1} B \subseteq B$.

Seja. $B_{1}=B+F \cdot a_{1}$. Novamente temos que $B \triangleleft B_{1}$ e $B_{1}{ }^{2} \subseteq B$. Logo, pelo Lema $2.12, \mathcal{R}\left(B_{1}\right)$ é nilpotente, o que é uma contradição, pois $B$ é maximal com essa propriedade. Portanto, $B=J$ e $\mathcal{R}(J)$ nilpotente.

Corolário 2.15 Sejam J uma álgebra de Jordan (não necessariamente de dimensão finita) e $a \in J$ com $a^{n}=0$. Então existe um $N=N(n)$ tal que $R_{a}^{N}=0$.

Demonstração: Seja $J_{0}=\operatorname{alg}\langle a\rangle$. Logo $J_{0}$ é de dimensão finita e nilpotente e, pelo Teorema 2.14 , temos $\mathcal{R}\left(J_{0}\right)$ é nilpotente. Assim $R^{J}\left(J_{0}\right)$ é nilpotente com índice de nilpotência $N$. 
Corolário 2.16 Seja J uma álgebra de Jordan de dimensão finita e nil. Então $J$ é nilpotente.

Demonstração: Como $J$ é de dimensão finita e nil, pelo Teorema 2.14, $R(J)$ é nilpotente. e como $J$ é comutativa, então $J$ é nilpotente. Em particular, se $\operatorname{dim} J<\infty$, então Nil $J$ é nilpotente.

Corolário 2.17 Cada álgebra de Jordan solúvel e de dimensão finita é nilpotente.

Os seguintes teoremas nos permite ter informação da decomposição das álgebras de Jordan nil-semisimples (Nil $J=0$ ).

Teorema 2.18 Seja J uma álgebra de Jordan de dimensão finita sobre um corpo de característica diferente de $2, J$ nil-semi-simples (Nil $J=0)$. Então $J$ é isomorfa a uma soma direta de álgebras de Jordan simples de dimensão finita e cada uma delas é de algum dos tipos $H(A, *), J(V, f), H\left(\mathcal{O}_{3}\right)$.

Demonstração: Ver [2].

Teorema 2.19 [Teorema de Wedderburn em álgebras de Jordan]

Seja $J$ uma álgebra de Jordan de dimensão finita sobre um corpo $\mathbb{F}$ de característica diferente de 2. Então, existe uma subálgebra $S$ de $J$ tal que $J=S+$ Nil $J$, onde Nil $J$ é o radical nil de $J$ e $S$ é soma de álgebras simples, $S \cong J / \mathrm{Nil} J$.

Demonstração: Ver Teorema de Albert, Penico, Taft em [2], Capítulo 7 $\S 6$.

Em virtude dos Teoremas 2.18 e 2.19, temos que o estudo das álgebras de Jordan de dimensão finita se reduz ao estudo da parte semi-simples e a 
parte nilpotente, mais o estudo da parte semi-simples se reduz ao estudo da estrutura das álgebras simples os quais conhecemos pelo Teorema 2.8. Assim, nosso objetivo é continuar com o estudo das álgebras simples e classificar estas segundo seu grau ou sua capacidade.

\subsection{Decomposição de Peirce}

\subsubsection{Por um idempotente $e$}

Seja $J$ uma álgebra de Jordan de dimensão finita e Nil $J \neq J$ ( $J$ não é nil). Assim, pela Proposição 1.28 , temos que $J$ possui um idempotente $0 \neq e=e^{2}$. Da identidade (2.9), temos que

$$
2 R_{a} R_{b} R_{a}+R_{a^{2} b}=R_{a^{2}} R_{b}+2 R_{a b} R_{a} \quad \forall a, b \in J .
$$

Tomando $a=b=e$, temos, de (2.13), que $2 R_{e}{ }^{3}-3 R_{e}{ }^{2}+R_{e}=0$, donde

$$
R_{e}\left(2 R_{e}-1\right)\left(R_{\epsilon}-1\right)=0
$$

Logo as raízes para o polinômio característico de $R_{e}$ são $\{0,1 / 2,1\}$, assim

$$
J=J_{1} \oplus J_{1 / 2} \oplus J_{0}
$$

onde

$$
J_{i}=\left\{x \in J / x R_{e}=i x\right\}, \quad i=0,1 / 2,1
$$

A decomposição de $J$ dada em (2.14) é chamada decomposição de Peirce de $J$ segundo o idempotente $e$.

\section{Observação 2.20}

1. De (2.2), temos que $\left(a^{2}, b, c\right)+2(a c, b, a)=0$. Portanto, para $x \in J$ e $a_{i} \in J_{i} \quad i=0,1$ verifica-se $\left(e, x, a_{i}\right)=\left(e^{2}, x, a_{i}\right)=-2\left(e a_{i}, x, e\right) \mathrm{e}$, 
pela comutatividade de $J$, temos que $-2\left(e a_{i}, x, e\right)=2\left(e, x, e a_{i}\right)$. Logo $(1-2 i)\left(e, x, a_{i}\right)=0$ mas $(1-2 i) \neq 0$ para $i=0,1$. Portanto

$$
\left(e, x, a_{i}\right)=0 \quad \forall x \in J, a_{i} \in J_{i}, \quad i=0,1 .
$$

2. Tomando $x=e$ na Identidade (2.15), temos que $\left(e, e, a_{i}\right)=0$.

Seja $i=1 / 2, \log \mathrm{o}$

$$
\left(e, e, a_{1 / 2}\right)=e^{2} a_{1 / 2}-e\left(e a_{1 / 2}\right)=\frac{1}{2} a_{1 / 2}-\frac{1}{4} a_{1 / 2}=\frac{1}{2} a_{1 / 2} \neq 0,
$$

Portanto, se $\left(e, e, a_{i}\right)=0$ então $a_{i} \in J_{0} \cup J_{1}$.

Lema 2.21 Seja $J$ uma álgebra de Jordan e e $\in J$ um idempotente. Considere a decomposição de Peirce de J segundo o idempotente e dada por (2.14). Então:

1. $J_{i}^{2} \subseteq J_{i} \quad i=0,1$.

2. $J_{0} J_{1}=0$.

3. $\left(J_{1 / 2}\right)^{2} \subseteq J_{0}+J_{1}$.

4. $\left(J_{0}+J_{1}\right) J_{1 / 2} \subseteq J_{1 / 2}$.

\section{Demonstração:}

1. Sejam $a_{i}, b_{i} \in J_{i}$ para $i=0,1$. Então

$$
e\left(a_{i} b_{i}\right)=\left(e a_{i}\right) b_{i}-\left(e, a_{i}, b_{i}\right)=i a_{i} b_{i}= \begin{cases}0 \in J_{0} & i=0 \\ a_{1} b_{1} \in J_{1} & i=1\end{cases}
$$

pois, pelo item 1 da Observação 2.20, temos que $\left(e, a_{i}, b_{i}\right)=0$. Portanto $J_{i}^{2} \subseteq J_{i}, \quad i=0,1$. 
2. Sejam $a \in J_{0}$ e $b \in J_{1}$. Então

$$
e(a b)=(e a) b-(e, a, b)=(e a) b=0
$$

Por outro lado,

$$
e(b a)=(e b) a-(e, b, a)=b a=a b=0
$$

Assim, $J_{0} J_{1}=0$.

3. Pelo item 2 da Observação 2.20, basta provar para todo $a, b \in J_{1 / 2}$ que $(e, e, a b)=0$. Temos, da Identidade $(2.3),(a, b, c d)=(a d, b, c)+$ $(a c, b, d)$. Fazendo $a=e=b, c=a, d=b$ temos

$$
(e, e, a b)=(e a, e, b)+(e b, e, a)=\frac{1}{2}(a, e, b)+\frac{1}{2}(b, e, a)=0 .
$$

Portanto $a b \in J_{0}+J_{1}$.

4. Seja $a \in J_{1 / 2}, b \in J_{0}+J_{1}$. Logo

$$
e(a b)=(e a) b-(e, a, b)=\frac{1}{2} a b \in J_{1 / 2}
$$

para todo $a \in J_{1 / 2}, b \in J_{0}+J_{1}$. Assim $\left(J_{0}+J_{1}\right) J_{1 / 2} \subseteq J_{1 / 2}$.

Definição $2.22 U m$ idempotente e em $J$ diz-se principal se $J_{0}(e)$ é nil.

Proposição 2.23 Toda álgebra de Jordan não nil e de dimensão finita contém um idempotente principal.

Demonstração: Pela Proposição 1.28, existe e idempotente em J. Se $e$ não é principal, então $J_{0}(e)$ não é nil. Portanto, existe um idempotente $f \in$ $J_{0}(e)$. Seja $e_{1}=e+f$. Portanto, $e_{1}$ é idempotente e $J_{1}(e) \subsetneq J_{1}\left(e_{1}\right)$. De fato, se $x \in J_{1}(e)$, então $e_{1} x=(e+f) x=e x=x$. Assim, temos $J_{1}(e) \subseteq J_{1}\left(e_{1}\right)$. Como $f \in J_{1}\left(e_{1}\right)$ e $f \notin J_{1}(e)$, então temos $\operatorname{dim} J_{1}(e)<\operatorname{dim} J_{1}\left(e_{1}\right)$, e como $J$ tem dimensão finita, o processo tem que parar. 
Vamos definir uma forma traço $(-,-)$ em $J$ e depois vamos verificar que o ideal radical associado com a forma traço $J^{\perp}$, está dado pelo máximo ideal solúvel. Com isto vamos ter outra caracterização do radical numa álgebra de Jordan de dimensão finita, $J^{\perp}=\{a \in J ;(a, b)=0, \quad \forall b \in J\}$.

Consideremos $(-,-): J \times J \longrightarrow \mathbb{F}$, uma forma bilinear simetrica em $J$ associada com a função traço $\operatorname{tr} R_{a}$, onde $\operatorname{tr} R_{a}$ é o traço da matriz de $R_{a}$. Definimos $(a, b)=\operatorname{tr} R_{a b}$ para todo $a, b \in J$.

Proposição 2.24 Seja J uma álgebra de Jordan, sobre um corpo de característica zero e $\mathrm{Nil} J=0$. A aplicação $\operatorname{tr} R_{a}$, é uma forma traço não degenerada.

Demonstração: $\quad \operatorname{Sejam~} a, b, c \in J$. Então

$$
\begin{array}{r}
(a b, c)-(a, b c)=\operatorname{tr} R_{a b \cdot c}-\operatorname{tr} R_{a \cdot b c}=\operatorname{tr} R_{a b \cdot c-a \cdot b c}=\operatorname{tr} R_{(a, b, c)} \\
=\operatorname{tr}\left[\left[R_{c}, R_{a}\right] R_{b}\right]=0
\end{array}
$$

Portanto, $\operatorname{tr} R_{a}$ é uma forma traço.

Temos que $J^{\perp}$ é um ideal de $J$ e, como Nil $J=0$, então, $J^{\perp}$ não é nil. Assim existe um $f \in J^{\perp}$ tal que $0 \neq f=f^{2}$. Seja $J=J_{1}(f)+J_{1 / 2}(f)+J_{0}(f)$ a decomposição de Peirce de $J$ segundo $f$. Seja $B_{1} \cup B_{1 / 2} \cup B_{0}=B$ uma base para $J$ (pois $J$ é de dimensão finita), onde $B_{i}$ é uma base para $J_{i}$, respectivamente.

$$
B_{1}=\left\{e_{1}, e_{2}, \ldots e_{k}\right\}, B_{1 / 2}=\left\{e_{1}^{\prime}, e_{2}^{\prime}, \ldots e_{s}^{\prime}\right\} \text { e } B_{0}=\left\{e_{1}^{\prime \prime}, e_{2}^{\prime \prime}, \ldots e_{r}^{\prime \prime}\right\} \operatorname{logo}
$$

$$
\left[\mathbf{R}_{\mathbf{f}}\right]_{\mathbf{B}}=\left[\begin{array}{l|l|l}
E_{k} & & \\
\hline & \frac{1}{2} E_{s} & \\
\hline & & 0_{r}
\end{array}\right]
$$

$(f, f)=\operatorname{tr} R_{f}^{2}=\operatorname{tr} R_{f}=k+\frac{1}{2} s \neq 0$, pois a característica de $\mathbb{F}$ é zero, a forma é não degenerada. 
Da Proposição anterior, temos que $\operatorname{tr} R_{a}$ é uma forma traço não degenerada em $J$, portanto $J^{\perp}$ (radical da forma traço) é um ideal de $J$, como vimos no primeiro Capítulo. Vamos a continuação verificar que $J^{\perp}$ é um nil-icleal, com isso teremos Nil $J \supseteq J^{\perp}$, logo verificamos a inclução contraria tendo assim a igualdade e portanto uma nova caracterização do radical Nil $J$ de $J$.

Teorema 2.25 [Albert] O radical nil $(\mathrm{Nil} J)$ de qualquer álgebra de Jordan $J$ de dimensão finita sobre um corpo de característica zero coincide com o radical $J^{\perp}$ da forma traço $(x, y)=\operatorname{tr} R_{x y}$.

Demonstração: Suponhamos que $J^{\perp}$ não é nil. Então, pela Proposição 1.28 , existe um idempotente $e \in J^{\perp}$. Como $\mathbb{F}$ tem característica zero, então $(e, e)=\operatorname{tr} R_{e} \neq 0$. Dado que $e \in J^{\perp},(e, x)=0$ para todo $x \in J$, o que é uma contradição. Portanto $J^{\perp}$ é nil. $\left(J^{\perp} \subseteq\right.$ Nil $\left.J\right)$.

Seja $x \in \operatorname{Nil} J$. Logo, para todo $y \in J$ temos que $x y \in$ Nil $J$ e, como Nil $J$ é nilpotente, então $R_{x y}$ é nilpotente $\left(\operatorname{tr} R_{x y}=0\right)$. Assin, temos que $x \in J^{\perp}$ para todo $x \in \operatorname{Nil} J$. Daqui e do anterior temos que Nil $J=J^{\perp}$.

Teorema 2.26 Seja J uma álgebra de Jordan de dimensão finita sobre $\mathbb{F}$ com característica de $\mathbb{F}$ zero e $\mathrm{Nil} J=0$. Então, $J=J_{1} \oplus J_{2} \oplus \cdots \oplus J_{k}$, onde cada $J_{i}$ é simples.

Demonstração: Pelo teorema de Dieudonné 1.6 e pela Proposição 2.24, temos $J=J_{1} \oplus \cdots \oplus J_{t}$, onde cada $J_{i}$ é simples.

Seja $J$ é uma álgebra de Jordan não nil e $e$ um idempotente (o qual sempre existe), $J_{1}+J_{1 / 2}+J_{0}$ a decomposição de Peirce de $J$ segundo $e$. Então pelas propriedades dadas no Lema 2.21, as propriedades da forma traço e a proposiçào anterior é fácil verificar o seguinte teorema. 
Teorema 2.27 Seja J uma álgebra de Jordan de dimensão finita sobre um corpo $\mathbb{F}$ de característica zero e $\mathrm{Nil} J=0$. Então, $J$ contém 1 .

Demonstração: $\quad$ ver [1], Capítulo 4, Teorema 4.7.

\subsubsection{Por um conjunto de idempotentes}

Proposição 2.28 Seja J uma álgebra de Jordan com 1 tal que $1=e_{1}+$ $\cdots+e_{n}$, onde os $e_{i}$ são idempotentes ortogonais. Então,

$$
J=\bigoplus_{i, j=1}^{n} J_{i j}
$$

onde $J_{i i}=J_{1}\left(e_{i}\right)$ e $J_{i j}=J_{1 / 2}\left(e_{i}\right) \cap J_{1 / 2}\left(e_{j}\right)$.

Demonstração: Sejam $e_{1}, e_{2}$ idempotentes ortogonais, $1=e_{1}+e_{2}$. Como $e_{1}$ é idempotente, então, pela decomposição de Peirce para $e_{1}$, temos que

$$
J=J_{1}\left(e_{1}\right) \oplus J_{1 / 2}\left(e_{1}\right) \oplus J_{0}\left(e_{1}\right)
$$

mas $J_{1 / 2}\left(e_{1}\right)=J_{1 / 2}\left(e_{2}\right)$ e $J_{1}\left(e_{1}\right)=J_{0}\left(\epsilon_{2}\right)$ portanto

$$
\begin{aligned}
J & =J_{1}\left(e_{1}\right) \oplus J_{1 / 2}\left(e_{1}\right) \oplus J_{0}\left(e_{1}\right) \\
& =J_{1}\left(e_{1}\right) \oplus J_{1 / 2}\left(e_{1}\right) \cap J_{1 / 2}\left(e_{2}\right) \oplus J_{1}\left(e_{2}\right) \\
& =J_{11} \oplus J_{12} \oplus J_{22}
\end{aligned}
$$

Assim o Teorema vale para $1=e_{1}+e_{2}$.

No caso geral, temos $1=e_{1}+\cdots+e_{n}=\underbrace{e_{1}+\cdots+e_{n-1}}_{e}+e_{n}=e+e_{n}$. Logo

$$
J=J_{1}(e) \oplus J_{1 / 2}(e) \oplus J_{1}\left(e_{n}\right)
$$

mas

$$
J_{1}(e)=\bigoplus_{i, j=1}^{n-1} J_{i j}
$$


Nós Resta demonstrar que $J_{1 / 2}\left(e_{n}\right)=J_{1 / 2}(e)$.

Seja $x \in J_{1 / 2}(e)$. Logo

$$
x=2 e x=2\left(e_{1} x+\cdots+e_{n-1} x\right)=4\left(e_{n} \cdot e_{1} x+\cdots+e_{n} \cdot e_{n-1} x\right) .
$$

Afirmação $\left(x e_{i}\right) e_{j}=\left(x e_{j}\right) e_{i}$.

Da Identidade (2.2), fixando $a, c$, temos $\left[R_{a^{2}}, R_{c}\right]+2\left[R_{a c}, R_{a}\right]=0$. Logo,

$$
\left[R_{e_{i}}, R_{e_{j}}\right]=\left[R_{e_{i}^{2}}, R_{e_{j}}\right]=2\left[R_{e_{i}}, R_{e_{i} e_{j}}\right]=0
$$

portanto a afirmação vale. Além disso, temos que

$$
\left(\left(x e_{i}\right) e_{j}\right) e_{i}=\left(x e_{i}, e_{j}, e_{i}\right)=-\frac{1}{2}\left(e_{i}{ }^{2}, e_{j}, x\right)=-\frac{1}{2}\left(e_{i}, e_{j}, x\right)=-\frac{1}{2} e_{i}\left(e_{j} x\right)
$$

Assim, $\left(x e_{i}\right) e_{j} \in J_{1 / 2}\left(e_{i}\right)$ e pela simetria temos que $\left(x e_{i}\right) e_{j} \in J_{1 / 2}\left(e_{j}\right)$. Portanto $\left(x e_{i}\right) e_{j} \in J_{i j}$ mas $J_{i j}=\sum_{i \neq j} J_{i j}$ é soma direta, portanto $x \in J_{i j}$ e $x e_{k}=0$ para todo $k$ diferente de $i, j$.

Corolário 2.29 Nas condiçôes da Proposição 2.28, temos:

i) $J_{i i}{ }^{2} \subseteq J_{i i}, \quad J_{i i} J_{j j}=0 \quad i \neq j$.

ii) $J_{i i} J_{i j} \subseteq J_{i j}, \quad J_{i j} J_{j k} \subseteq J_{i k}$

iii) $J_{i i} J_{k l}=J_{i j} J_{k l}=0, \quad J_{i j}{ }^{2} \subseteq J_{i i}+J_{j j}$ para $i, j, k, l$ distintos

Demonstração: Análogo ao feito no Lema 2.21

\section{5 Álgebras de Jordan reduzidas}

Definição 2.30 Seja $J$ uma álgebra de Jordan. Um idempotente $e \in J$ dizse absolutamente primitivo se, para todo elemento $x \in J_{1}(e), x=\alpha e+\eta$, onde $\alpha \in \mathbb{F}$ è $\eta$ é nilpotente. 
Lema 2.31 Seja J uma álgebra de Jordan de dimensão finita sobre $\mathbb{F}$. Então:

1) Todo idempotente absolutamente primitivo em $J$ é primitivo.

2) A recíproca vale se o corpo $\mathbb{F}$ é algebricamente fechado.

\section{Demonstração:}

1) Seja $e$ absolutamente primitivo em $J$ e suponhamos que $e=e_{1}+e_{2}$, com $\epsilon_{1}, e_{2}$ idempotentes ortogonais em $J_{1}$. Mas $e_{1}, e_{2}$ pertencem a $J_{1}$, pois $e e_{1}=e_{1}$ e $e e_{2}=e_{2}$. Temos, assim, que $e_{1}=\alpha e+\eta$, onde $\eta$ é nilpotente e $0 \neq \alpha \in \mathbb{F}$. Então, $e_{1}=e_{1}{ }^{2}=(\alpha e+\eta)^{2}=(\alpha)^{2} e+2 \alpha \eta+\eta^{2}=\alpha e+\eta$. Onde $2 \alpha \eta+\eta^{2}$ é nilpotente. Logo $\alpha^{2}=\alpha=1$. Assim, $e_{1}=e+\eta=$ $e+2 \eta+\eta^{2}$ donde $\eta=-\eta^{2}=0$. Portanto $e_{1}=e$. Do mesmo jeito provamos que $e_{2}=e$. Logo e é primitivo em $J$.

2) Seja $\mathbb{F}$ um corpo algebricamente fechado, e primitivo, $a \in J_{1}(e)$, e $f(x)$ o polinômio minimal de $a(f(a)=0)$.

Se $f(x)=f_{1}(x) f_{2}(x)$ com $\operatorname{mdc}\left(f_{1}, f_{2}\right)=1$. Então pelo Teorema de Bezout existem polinômios $s(x), t(x)$ tal que $s(x), f_{1}(x)+t(x) f_{2}(x)=1$. Como é idempotente em $J_{1}(e)$ então, $s(a) f_{1}(a)+t(a) f_{2}(a)=e$. Sejam $s(a) f_{1}(a)=e_{1}$ e $t(a) f_{2}(a)=e_{2}$. Logo $e_{1} e_{2}=0, e_{1}^{2}=e_{1}, e_{2}^{2}=e_{2}$. Se $e_{1}=e$ então $s(a) f_{1}(a)=e$. Portanto $s(a) f_{1}(a) f_{2}(a)=f_{(}(a)=0$ donde $f(x)=(x-\alpha)^{n}$ e $f(x)=(x-\alpha e)^{n}=0$. Assim $a=\alpha e+\eta, \quad \eta=a-\alpha e$.

Definição 2.32 Uma álgebra $A$ diz-se reduzida se contém 1 e existem idempotentes absolutamente primitivos ortogonais $e_{1}, \ldots, e_{n}$, tais que $1=e_{1}+$ $\cdots+e_{n}$.

Pode-se verificar que a quantidade $n$ de elementos idempotentes é invariante.

Este número é chamado grau de $A$. 
Teorema 2.33 Toda álgebra de Jordan com 1 sobre um corpo algebricamente fechado é reduzida.

\section{Demonstração:}

i) Se 1 é primitivo então o Teorema vale.

ii) $1=e_{1}+e_{2}$ não é primitivo. Se $e_{1}, e_{2}$ foram primitivos, então o Teorema vale. Caso contrário, temos que $e_{1}=e_{1_{1}}+e_{1_{2}}$ e $e_{2}=e_{2_{1}}+e_{2_{2}}$, onde os $e_{i_{j}}$ são ou não primitivos. O processo tem que parar pois $J$ é de dimensão finita. Assim, vamos ter que $1=e_{1}+\cdots+e_{n}$ primitivos, mas $\mathbb{F}$ é algebricamente fechado, portanto os $e_{i}$ são absolutamente primitivos.

\subsubsection{Triplo produto de Jordan e inversos}

Seja $J$ uma álgebra de Jordan, denotamos $a \cdot b \cdot c:=(a \cdot b) \cdot c$, o triplo produto de Jordan em $J$ é definido por

$$
\{a b c\} \equiv a \cdot b \cdot c+b \cdot c \cdot a-a \cdot c \cdot b
$$

Fixados $a, b$ em $J$ define-se a aplicação linear $x U_{a, b}=\{a x b\}$.

Observação 2.34 Propriedades do operador $U_{a, b}$

1. $U_{a, b}=R_{b} R_{a}+R_{a} R_{b}-R_{a b}$. Em particular para $b=a$ temos que $U_{a} \equiv$ $U_{a, a}=2 R_{a}{ }^{2}-R_{a^{2}}$, de onde $U_{a} \in \operatorname{alg}\left\langle R_{a}, R_{a^{2}}\right\rangle$

2. $U_{\alpha a}=\alpha^{2} U_{a}, U_{a, b}=\frac{1}{2}\left(U_{a+b}-U_{a}-U_{b}\right)$.

3. Dada a simetria em (2.18) temos

$$
\{a b c\}=\{c b a\}, \quad \text { e } \quad\{1 a b\}=\{a \cdot 1 b\}=\{a b 1\}=a \cdot b .
$$


4. Em álgebras especiais de Jordan, temos que o triplo produto de Jordan $\{a b c\}=a b c+c b a$, e vale a Identidade $\{\{a b a\} c\{a b a\}\}=\{a\{b\{a c a\} b\} a\}$. Logo, pelo Teorema de Macdonald 2.3, temos que $U_{b U_{a}}=U_{a} U_{b} U_{a}$.

Definição 2.35 Seja $J$ uma álgebra de Jordan com 1. Dizemos que a em J é invertivel se existe um $b \mathrm{em} J$ tal que $a \cdot b=1$ e $a^{2} \cdot b=a$

Proposição 2.36 Seja $J$ uma álgebra de Jordan com 1, e a em J. Então.

1. Se b é um inverso para a, então a é um inverso para b.

2. a é invertível se, e somente se, $U_{a}$ é invertivel.

3. $U_{a}^{-1}=U_{a^{-1}}$.

4. $a, b$ são invertiveis se, e somente se, $\{b a b\}=a U_{b}$ é invertivel.

Demonstração: $\quad$ ver [2], Capítulo $1 \S 11$, Teorema 13.

Lema 2.37 (McCrimmon) Seja $J$ uma álgebra de Jordan com 1 e $u \in J$, $u=1-4 \eta$ com $\eta$ nilpotente. Então existe um $w \in J$, nilpotente tal que $u=1-4 \eta=(1-2 w)^{2}$.

Demonstração: temos que encontrar um $w$ nilpotente tal que $w-w^{2}=$ $z$. Como $z$ é nilpotente, existe $n$ tal que $z^{n}=0$. Seja $w=\sum_{i=1}^{n-i} \beta_{i} z^{i}$. Comparando os coeficientes de cada potência em $z$, temos que $\beta_{1}=1 \mathrm{e}$ $\beta_{i}=\sum_{k=1}^{i-1} \beta_{k} \beta_{i-k}$.

Teorema 2.38 (Albert-Jacobson-McCrimmon) Seja J uma álgebra de Jordan com 1, tal que para todo $x$ em $J, x=\alpha \cdot 1+\eta$ com $\eta$ nilpotente. Então $J=\mathbb{F} \cdot 1+\mathrm{Nil} J$. 
Demonstração: Basta demonstrar que a soma de elementos nilpotentes é nilpotente. Por contradição, suponhamos $\eta_{1}, \eta_{2}$ são nilpotentes e $\eta_{1}+\eta_{2}$ não é nilpotente. Como $\eta_{1}+\eta_{2} \in J$ então $\eta_{1}+\eta_{2}=\alpha \cdot 1+\omega \operatorname{com} \omega$ nilpotente.

Sem perda de generalidade, vamos supor $\alpha=1$ e $\eta_{1}+\eta_{2}=1-4 z \operatorname{com} z$ nilpotente. Pelo Lema de McCrimmon 2.37, temos que existe $\nu$ nilpotente tal que $1-4 z=(1-2 \nu)^{2}=\mu^{2}$ e $\mu$ invertivel, pois

$$
1=1-(2 z)^{s}=(1-2 z)\left(1+2 z+\cdots+(2 z)^{s-1}\right)
$$

donde $(1-2 z)^{-1}=1+2 z+\cdots+(2 z)^{s-1}$. Assim, temos que $U_{\mu}$ é invertível, e $\left(\eta_{1}+\eta_{2}\right) U_{\mu}^{-1}=\mu^{2} U_{\mu}^{-1}=1$. Pela Proposição 2.36-4, temos $\left(\eta_{1}+\eta_{2}\right)$ invertível, o que é uma contradição. Portanto, $\left(\eta_{1}+\eta_{2}\right)$ é nilpotente.

Sejam $J$ uma álgebra de Jordan reduzida, $E=\left\{e_{1}, e_{2}, \ldots e_{n}\right\}$ conjunto de idempotentes ortogonais em $J$ e $1=e_{1}+\cdots+e_{n}$. Pelo Teorema 2.28, temos que

$$
J=\bigoplus_{i, j=1}^{n} J_{i j}=\bigoplus_{i=1}^{n} J_{i i}+\bigoplus_{\substack{i, j=1 \\ i<j}}^{n} J_{i j}
$$

e como os $e_{i}$ são absolutamente primitivos, então $J_{i i}=\mathbb{F} \cdot e_{i}+\operatorname{Nil} J_{i i}$. Assim,

$$
J=\sum_{i=1}^{n}\left(\mathbb{F} \cdot e_{i}+\text { Nil } J_{i i}\right)+\sum_{\substack{i, j=1 \\ i<j}}^{n} J_{i j} .
$$

Para todo $a \mathrm{em} J, a$ pode ser decomposto de modo único como

$$
a=\sum_{i=1}^{n}\left(\alpha_{i} \cdot e_{i}+\eta_{i}\right)+\sum_{\substack{i, j=1 \\ i<j}}^{n} a_{i j} .
$$

Seja $t_{\mathrm{E}}$ a aplicação dada pela decomposição de $a \in J$ e pelo conjunto $E$, definida por $\mathrm{t}_{\mathrm{E}}(a)=\mathrm{t}_{\mathrm{E}}\left(\sum_{i=1}^{n}\left(\alpha_{i} \cdot e_{i}+\eta_{i}\right)+\sum_{\substack{i, j=1 \\ i<j}}^{n} a_{i j}\right)=\sum_{i=1}^{n} \alpha_{i}$.

Observação 2.39 As seguintes são algumas propriedades fornecidas pela aplicação $\mathrm{t}_{\mathrm{E}}$. 
1. $\mathrm{t}_{\mathrm{E}}\left(e_{i}\right)=1, \quad \forall i$.

2. $t_{E}(1)=n$.

3. $\mathrm{t}_{\mathrm{E}}\left(a_{i j}\right)=0 \quad$ se $\mathrm{i} \neq \mathrm{j}$.

4. $\mathrm{t}_{\mathrm{E}}\left(a_{i}\right)=0 \quad$ sempre que $\mathrm{a}_{\mathrm{i}} \in \mathrm{Nil} \mathrm{J}_{\mathrm{ii}}$.

Consideremos a forma bilinear induzida por $t_{E}$,

$$
\begin{aligned}
\mathrm{t}_{\mathrm{E}}(,): J \times J & \longrightarrow \mathbb{F} \\
a, b & \mapsto \mathrm{t}_{\mathrm{E}}(a, b)=\mathrm{t}_{\mathrm{E}}(a b)
\end{aligned}
$$

a qual chamaremos forma bilinear associada $a \mathrm{t}_{\mathrm{E}}$.

Proposição 2.40 A forma $\mathrm{t}_{\mathrm{E}}(a, b)$ é uma forma traço.

Demonstração: $\quad$ Ver Capítulo 5 \& 4 de [2]

Corolário 2.41 Seja J uma álgebra de Jordan reduzida e $J^{\perp}$ o ideal radical de $\mathrm{t}_{\mathrm{E}}$. Então:

1. $\left(J^{\perp}\right)_{i i}=\operatorname{Nil} J_{i i}$.

2. $\left(J^{\perp}\right)_{i j}=\left\{z_{i j} \in J_{i j}: z_{i j} J_{i j} \subseteq \mathrm{Nil} J_{i i}+\mathrm{Nil} J_{j j}\right\}$.

Corolário 2.42 Seja $J$ reduzida e simples. Então $J=\sum_{i=1}^{n} \mathbb{F} e_{i}+\sum_{j>i=1}^{n} J_{i j}$. Além disso, temos que, para todo elemento não nulo $a_{i j} \in J_{i j}$, existe $b_{i j} \in J_{i j}$ tal que $a_{i j} b_{i j}$ é invertivel em $J\left(e_{i}+e_{j}\right)$. 


\subsection{Teorema de Coordenatização}

Definição 2.43 Dois idempotentes ortogonais $e_{i}, e_{j}$ são ditos conectados se existe $a_{i j} \in J_{i j}$ tal que $a_{i j}$ é invertível em $J\left(e_{i}+e_{j}\right)$. Dois idempotentes ortogonais $e_{i}, e_{j}$ são ditos fortemente conectados se existe $a_{i j} \in J_{i j}$ tal que $a_{i j}{ }^{2}=e_{i}+e_{j}$.

É claro da definição anterior que dados $e_{i}, e_{j}$ fortemente conectados então $J_{i j} \neq 0$. Os seguintes Lemas, nos permitem ter informação da estrutura das álgebras de Jordan reduzidas simples em relação de elementos conectados ou fortemente conectados. Para uma demonstração dos mesmos recomendamos ler [2].

Lema $2.44 J_{i j} \neq 0$ se, e somente se, $J_{i j}$ tem um elemento invertível.

Lema 2.45 A relação conectados e fortemente conectados são relações transitivas.

Lema 2.46 Seja J uma álgebra de Jordan reduzida e simples, $1=\sum_{i=1}^{n} e_{i}$. Então, para todo par $i, j$ existe $a_{i j} \in J_{i j}$ tal que $a_{i j}{ }^{2}=e_{i}+e_{j}$.

\section{Álgebra de matrizes}

Seja $D$ uma álgebra com 1 sobre um corpo $\mathbb{F}$ com característica diferente de 2 e $d \mapsto \bar{d}$ uma involução em $D$. Consideremos $D_{n}$ a álgebra de matrizes de ordem $n \times n$ com entradas em $D$ e onde $e_{i j}$ representa a matriz unidade usual, com 1 na posição $i, j$ e zero nas outras. Um elemento $x \in D$ é identificado em $D_{n}$ pela matriz diagonal que só tem $x$ em cada entrada da diagonal. Seja $a=\left\{a_{1}, a_{2}, \ldots a_{n}\right\}$ um subconjunto do núcleo de $D$, talque cada $a_{i}$ é invertivel e $a_{i}=\bar{a}_{i}$. 
Seja $*: D_{n} \longrightarrow D_{n}$ a involução induzida em $D_{n}$ pela involução - de $D$, com seguinte aplicação:

$$
(x[i j])^{*}=\left(x e_{i j}+x e_{i j}\right)^{*}=x e_{i j}+\left(a_{j}^{-1} \bar{x} a_{i}\right) e_{j i}
$$

A involução $*$ diz-se involução estandard quando $a=\{1,1, \cdots, 1\}$. Neste caso, $(x[i j])^{*}=x e_{i j}+\left(x e_{i j}\right)^{*}=x e_{i j}+\bar{x} e_{j i}$, para todo $x \in D$.

Lema 2.47 As seguintes afirmações são regras de multiplicação na álgebra de matrizes.

i) $2 x[i j] \cdot y[j k]=(x y)[i k]$ para $i, j, k$ diferentes $e$ xy representa o produto na álgebra $D$.

ii) $2 x[i i] \cdot y[i j]=(x y+\bar{x} y)[i j]$ para $i \neq j$.

iii) $2 x[i j] \cdot y[j i]=(x y)[i i]+(y x)[j j], \quad i \neq j$.

iv) $2 x[i i] \cdot y[i i]=((x+\bar{x})(y+\bar{y})[i i]$.

v) $x[i j] \cdot y[k l]=0, \quad\{i, j\} \cap\{l, k\}=\emptyset$.

Demonstração: $\quad$ ver [2], Capítulo $3 \S 2$.

Seja $J$ uma álgebra de Jordan e $e_{i}$ um elemento idempotente ortogonal. Associamos com $e_{i}$ o elemento $\frac{1}{2}[i i]=e_{i i}$ em $J_{n}$ e $u_{i j} \in J_{n}$ representa o elemento $1[i j]$.

Lema 2.48 Seja $J$ uma álgebra de Jordan reduzida e simples, $1=e_{1}+\cdots+$ $e_{n}, u_{i j} \in J_{i j}$, com $i \neq j$ tal que $u_{i j}^{2}=e_{i}+e_{j}, p_{i j}=1-e_{i}-e_{j}+u_{i j}=$ $\sum_{k \neq i, j} e_{k}+u_{i j}$. Consideremos a aplicação $U_{(i j)}=U_{p_{i j}}, R_{i j}=R_{u_{i j}}$, então $U_{i j} \in \operatorname{Aut} J, U_{i j}{ }^{2}=1 e$

1. $U_{i j}$ permuta $e_{i}, e_{j}, J_{i i}$ e $J_{\mathrm{jj}}, \mathrm{e}_{\mathrm{ij}} \mathrm{U}_{\mathrm{ij}}=\mathrm{J}_{\mathrm{ji}}$ 
2. $\left.U_{i j}\right|_{J_{k l}}=1_{J_{k l}}$ quando $k, l \neq i, j$

3. $U_{i j}$ permuta $J_{i k}$ e $J_{j k}$ se $k \neq i, j$ e $\left.U_{i j}\right|_{J_{i k}}=\left.2 R_{i j}\right|_{J_{i k}} ;\left.U_{i j}\right|_{J_{j k}}=\left.2 R_{i j}\right|_{J_{j k}}$. Demonstração: $\quad$ ver [2], Capítulo $3 \S 3$, Lema 1.

Lema 2.49 Seja $\Sigma=$ gr $<U_{i j}>\leq$ Aut $J$ então existe um isomorfismo $\phi \mapsto U_{\phi}$ do grupo simétrico Sym $n$ sobre $\Sigma$ tal que $(i j) \mapsto U_{i j}$. Temos para cada $i, e_{i} U_{\phi}=e_{\phi(i)}$. Ainda mais, se $\phi, \phi^{\prime} \in \operatorname{Sym} n$ e $\phi(i)=\phi^{\prime}(i), \phi(j)=\phi^{\prime}(j)$ então $U_{\phi}$ e $U_{\phi^{\prime}}$ agem igualmente sobre $J_{i j}$ para todo $i, j$.

Demonstração: $\quad$ ver [2], Capítulo 3, § 3, Lema 2.

Definição 2.50 Uma álgebra $D$ com involução - é dita uma álgebra de composição se:

1. D é uma álgebra alternativa com 1 .

2. $x \bar{x}=f(x) 1=\bar{x} x$ onde $f(x)$ é uma forma quadrática cuja forma bilinear simétrica associada $f(x, y)=\frac{1}{2}(f(x+y)-f(x)-f(y))$ é não degenerada.

Lema 2.51 Seja $D=J_{1 / 2}$. Definimos uma multiplicação em $D$ por

$$
x * y=2 x U_{23} \cdot y U_{13}
$$

$D$ é uma álgebra de composição com $1_{D}=u_{12}$ e involução $d \mapsto \vec{d}=d U_{12}$

Demonstração: $\quad u_{12}$ é unidade. De fato, temos

$$
x u_{12}=2 x U_{23} u_{12} U_{13}=2 x U_{23} u_{23}=\left(x U_{23}\right) 2 R_{23}=x U_{23} U_{23}=x
$$

Logo $u_{12}$ é unidade à direita. De modo análogo se prova que é unidade a esquerda. 
Vamos mostrar que $D$ tem uma involução - . Lembremos das propriedades do grupo simétrico que $\left(\begin{array}{lll}2 & 3\end{array}\right)\left(\begin{array}{ll}1 & 2\end{array}\right)=\left(\begin{array}{lll}1 & 2\end{array}\right)\left(\begin{array}{lll}1 & 3\end{array}\right)$, e $\left(\begin{array}{lll}1 & 3\end{array}\right)\left(\begin{array}{ll}1 & 2\end{array}\right)=\left(\begin{array}{lll}1 & 2\end{array}\right)\left(\begin{array}{ll}2 & 3\end{array}\right)$. Portanto, $U_{23} U_{12}=U_{12} U_{13}$ e $U_{13} U_{12}=U_{12} U_{23}$.

Sejam $x, y \in D$, Da observação anterior temos

$$
\begin{aligned}
& \overline{x * y}=2\left(x U_{23} \cdot y U_{13}\right) U_{12}=2 x U_{23} U_{12} \cdot y U_{13} U_{12}= \\
& 2 x U_{12} U_{13} \cdot y U_{12} U_{23}=2 \bar{x} U_{13} \cdot \bar{y} U_{23}=2 \bar{y} U_{23} \cdot \bar{x} U_{13}=\bar{y} * \bar{x}
\end{aligned}
$$

portanto - é uma involução em $D$.

Falta demonstrar que $D$ é álgebra de composição. Sejam $x, y \in D, \operatorname{logo}$

$$
\begin{aligned}
& f_{12}(x * y)=f_{12}\left(2 x U_{23} \cdot \bar{y} U_{13}\right)=f_{12}\left(2.2 x u_{23} 2 y u_{13}\right)=f_{13}\left(2 x u_{23}\right) f\left(2 x u_{13}\right)= \\
& f_{12}(x) f_{23}\left(u_{23}\right) f_{12}(y) f_{13}\left(u_{13}\right)=f_{12}(x) f_{12}(y) .
\end{aligned}
$$

portanto $D$ é álgebra de composição.

Teorema 2.52 Seja J uma álgebra de Jordan reduzida simples, com $1=$ $e_{1}+\cdots+e_{n}$, e $n>2$. Então, $J$ é isomorfa a $H\left(D_{n}, *\right)$ onde $D$ é uma álgebra de composição de dimensão n com involução - .

Demonstração: Como $J$ é reduzida simples, então para cada par" $i, j$ existe $a_{i j} \in J_{i j}$ talque $a_{i j}{ }^{2}=e_{i}+e_{j}$.

Seja $D=J_{1 / 2}$ e $x * y=2 x U_{23} y U_{13}$. Para todo $i, j$ definimos a aplicação $\psi: D \longrightarrow J_{i j}$ da seguinte maneira.

1. Se $i \neq j$ tome $\phi \in \operatorname{Sym}(n)$ tal que $1 \phi=i, 2 \phi=j$, e para $x \in$ $D$ definimos $\psi(x):=x_{i j}=x U_{\phi}$. Observe-se que a função está bem definida, pelo Lema 2.49 .

2. Se $i=j$, tome $\phi \in \operatorname{Sym}(n)$ tal que $1 \phi=i$. Definimos $\psi(x):=x_{i i}=$ $2 x R_{12} R_{e_{1}} U_{\phi}$

Seja $i \neq j, \bar{x}_{i j}=\bar{x} U_{\phi}=x U_{12} U_{\phi}=x U_{\phi^{\prime}}=x_{i j}$. Assim, $x_{i j}=\bar{x}_{i j}$.

Como $u_{12}{ }^{2}=e_{1}+e_{2}$, então $R_{12}{ }^{3}=R_{u_{12}}{ }^{3}=\dot{R}_{u_{12}}$ sobre $J_{1}\left(e_{1}+e_{2}\right) \mathrm{e}$ 


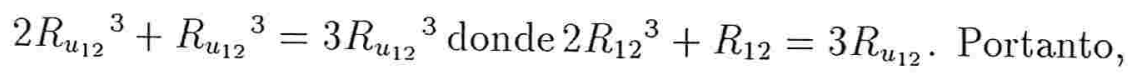

$$
u_{12} R_{12}=\left(2 R_{12}-R_{u_{12}{ }^{2}}\right) R_{12}=2 R_{12}^{3}-R_{12}=R_{12}
$$

sobre $J_{1}\left(e_{1}+e_{2}\right)$.

Para todo $x \in J_{12}$, temos que $\bar{x}_{i i}=2 x U_{12} R_{12} R_{e_{1}} U_{\phi}=x_{i i}$ donde $\overline{x_{i i}}=x_{i i}$.

Para $i \neq j$ e para todo $\phi^{\prime}$ temos $x_{i j} U_{\phi^{\prime}}=x U_{\phi} U_{\phi^{\prime}}=x U_{\phi \phi^{\prime}}$, onde $\phi^{\prime}$ age da mesma maneira sobre os índices de $x_{i j}$, temos que $x_{i i} U_{\phi^{\prime}}=x_{i \phi \cdot j \phi^{\prime}}$. Em particular, as aplicações $D \longrightarrow J_{i j}$ são injetoras.

Denotamos $u_{12}$ por 1 (identidade em $D$ ). Então $1_{i i}=2 u_{12}{ }^{2} e_{1} U_{\phi}=2 e_{1}$. Para $j>1$, temos

$$
\begin{aligned}
& 1_{i j}=u_{12} U_{2 j}=2 u_{12} u_{2 j}=u_{1 j} \\
& 1_{12}=u_{12} \\
& 1_{i j}=u_{i j}, \forall i, j
\end{aligned}
$$

Para provar que $J \cong H\left(D_{n}\right)$ definimos a aplicação linear

$$
\begin{aligned}
\psi: H\left(D_{n}\right) & \longrightarrow J \\
x[i j] & \mapsto x_{i j} \in J
\end{aligned}
$$

a qual é um isomorfismo nos espaços vetoriais. Resta demonstrar que $\psi$ é um isomorfismo de álgebras. Tendo em conta as regras de multiplicação dadas no Lema 2.47 temos que $\psi$ é um isomorfismo de álgebras.

Proposição 2.53 Seja $(D,-)$ uma álgebra com involução $d \mapsto \bar{d}, J=$ $H\left(D_{n}, *\right)$ a álgebra com multiplicação $a \cdot b=\frac{1}{2}(a b+b a)$ e assuma que $J$ é de Jordan. Então,

1. Se $n>3, D$ é associativa.

2. Se $n=3$ então $D$ é alternativa tal que $a+\bar{a}$ pertence ao centro associativo de D. 


\section{Demonstração:}

1. Suponhamos que $n>3, i, j, k$ diferentes e $1=\sum_{i=1}^{n} e_{i}, \quad e_{i}=\frac{1}{2}[i i]=$ $e_{i i}$.

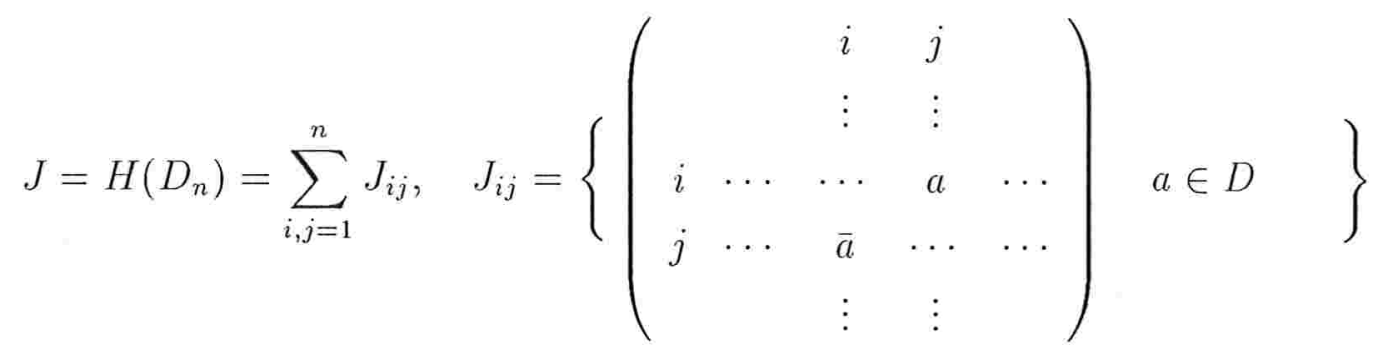

$\forall x \in D$, tem-se $x[i j] \in J_{i j}$.

Em $J$, consideremos

$$
\left(x_{i j}, y_{j k}, z_{k l}\right)=2\left(e_{i} x_{i j} y_{j k}, z_{k l}\right)=-2\left(e_{i} z_{k l} y_{j k}, x_{i j}\right)-2\left(x_{i j} z_{k l}, y_{j k}, e_{i}\right)=0
$$

Sejam $x, y, z \in D$. Logo

$$
\begin{aligned}
& 0=4(x[i j], y[j k], z[k l])=2(2(x[i j], y[j k], z[k l])-\cdots \\
& =(x y) z[i l]-x(y z)[i l]=(x, y, z)[i l]
\end{aligned}
$$

Assim, $(x, y, z)=0$ e, portanto, a álgebra é associativa.

2. Para $n=3$. Sejam $i, j, k$ diferentes e $\left(x_{i j}, y_{j k}, z_{k k}\right) \in J$. Logo

$$
\left(x_{i j}, y_{j k}, z_{k k}\right)=\left(x_{i j}, y_{j k}, z_{k k} e_{k}\right)=-\left(z_{k k}, y_{j k}, x_{i j}\right)-\left(e_{k}, y_{j k}, z_{k k} x_{i j}\right)=0
$$

Assim,

$$
\begin{aligned}
& 0=4(x[i j], y[j k], z[k k])=2(x y)[i k] \cdot z[k k]-2 x[i j](y(z+\bar{z}))[i k]= \\
& (x y)(z+\bar{z})-x(y(z+\bar{z}))[i k] \text { portanto }(x, y, z+\bar{z})=0 \text { em } D .
\end{aligned}
$$

Além disso, $4(x[i j] y[j k]) z[j k]=4(x[i j] z[j k]) y[i j]+4(y[i j] z[j k]) x[i j]$ mas $2((x \bar{y})[i i]+(\bar{y} x)[j j]) z[j k]=(\bar{y} x+\overline{\bar{y} x}) z[j \dot{k}] \log \mathrm{o}$ 


$$
\begin{aligned}
& 4(x[i j] z[j k]) y[i j]=2(x z)[i k] y[i j]=2(\overline{x z}[k i] y[i j])=(\overline{x z} \cdot y)[k j] \\
& 4(y[i j] z[j k] x[i j])=\cdots=(\overline{y z} \cdot x)[k j] .
\end{aligned}
$$

Conjugando a segunda parte, temos $(\bar{y} x+\bar{x} y) z[j k]=(\bar{y} \cdot x z+\bar{x} \cdot y z)[j k]$. Portanto, $(\bar{y}, x, z)+(\bar{x}, y, z)=0$. Fazendo $x=y$, temos $(\bar{x}, x, z)=0$. $\operatorname{Assim} \overline{(a, b, c)}=\overline{a b \cdot c}-\overline{a \cdot b c}=\cdots=-(\bar{c}, \bar{b}, \bar{a}) \mathrm{e}, \operatorname{como}(x, y, z+\bar{z})=0$, resulta pelas conjugadas que $(z+\bar{z}, \bar{y}, \bar{x})=0$ para todo $x, y, z$, onde $(z+\bar{z}, x, y)=0$, para todo $x, y$, mas temos, $(x+\bar{x}, \cdot \cdot \cdot)=0$. Logo, $0=(\bar{x}, x, z)=-(x, x, z)$, o que nos leva a concluir que $D$ é alternativa à esquerda. Do mesmo jeito,'se prova que $D$ é alternativa à direita.

\section{7 Álgebras de Jordan Simples}

Seja $J$ uma álgebra de Jordan com unidade, reduzida e simples, $1=\sum_{i=1}^{n} e_{i}$, e $E=\left\{e_{i}\right\}_{1}^{n}$ conjunto reduzido de idempotentes absolutamente primitivos ortogonais. Seja a decomposição de Peirce para $J$ dada por $J=\sum_{i=1}^{n} \mathbb{F} \cdot e_{i}+$ $\sum_{j>i=1}^{n} J_{i j}$ e $t_{\mathrm{E}}$ a forma traço bilinear associada dada pela Proposição 2.40 . Como vimos anteriormente, no Teorema de Albert 2.25, o ideal radical $J^{\perp}$ associado com a forma traço é nil e pelo Corolário 2.41, para $i \neq j$ temos que $J_{i j}^{\perp} \cap J_{i j}=\left\{z_{i j} \in J_{i j} / z_{i j} J_{i j} \subset \mathrm{Nil} J_{i i}+\mathrm{Ni} \_J_{j j}\right\}$. Como $J^{\perp}$ é um ideal de $J$, então pela simplicidade de $J$ temos que $J^{\perp}=J$ ou $J^{\perp}=0$. Como $\mathrm{t}_{\mathrm{e}}\left(e_{1}, e_{1}\right)=\mathrm{t}_{\mathrm{e}}\left(e_{1}{ }^{2}\right)=1$ então $J^{\perp} \neq J$ portanto $J^{\perp}=0$ e assim $\mathrm{t}_{\mathrm{e}}$ é não degenerada. Suponhamos que para algum $i \neq j J_{i j} \neq 0$. Seja $a_{i j} \in J_{i j}$ tal que $a_{i j}^{2} \neq 0$, podemos supor que $a_{i j}^{2}=\alpha_{i j}\left(e_{i}+e_{j}\right)$, com $\alpha_{i j} \in \mathbb{F}$, portanto, $a_{i j}$ é invertível em $J_{i i}+J_{j j}+J_{i j}$, e assim, $e_{i}, e_{j}$ são conectados. Continuando com um razonamiento análogo vamos ter que todos os $e_{i}$ são conectados.

Finalmente vamos considerar a estrutura das álgebras de Jordan de dimensão finita segundo seu grau.

1. $n=1, J=\mathbb{F} \cdot e_{1} \cong \mathbb{F}$. 
2. $n=2$. Seja $V=\mathbb{F}\left(e_{1}-e_{2}\right)+J_{12}$. Se $a_{12} \in J_{12}$ então $a_{12}^{2}=\alpha\left(e_{1}+e_{2}\right)=$ $\alpha \cdot 1$ e $\left(e_{1}-e_{2}\right) a_{12}=0$. Logo, se $x=\beta\left(e_{1}-e_{2}\right)+a_{12}$ então, $x^{2}=f(x) \cdot 1$ onde, $f(x)=\beta^{2}+\alpha \in \mathbb{F}$. Se segue que $f(x)$ é uma forma quadrática em $V$ e que $J=\mathbb{F} \cdot 1+V$ é uma álgebra de Jordan de forma quadrática. Como $\operatorname{dim} V>1$ e a forma $f$ é não degenerada, então $J$ é uma álgebra de Jordan simples. Note que $f\left(e_{1}-e_{2}\right)=1$, assim $f$ representa a unidade em $J$.

3. $n \geq 3$. Do Teorema de Coordenatização temos que $J$ é uma álgebra de matrizes.

Agora estamos em condições de enunciar um Teorema que nos permite ter uma classificação completa das álgebras de Jordan simples de dimensão finita, segundo seu grau.

Teorema 2.54 (Albert) Qualquer álgebra de Jordan simples de dimensão finita é uma álgebra de algum dos seguintes tipos

1. $J=\mathbb{F} \cdot 1$.

2. $J=\mathbb{F} \cdot 1+V$ álgebra de Jordan de forma simétrica não degenerada $f$ sobre $V e \operatorname{dim} V>1$.

3. $J \cong H\left(D_{n}, *\right), n \geq 3$. Onde $(D,-)$ é uma álgebra de composição de dimensão 1,2, ou 4 se $n \geq 4$ e de dimensão 1 ,2, 毛 ou 8 se $n=3$. 


\section{Capítulo 3}

\section{SUPERÁLGEBRAS}

Uma álgebra $A=A_{0} \dot{+} A_{1}$ que satisfaz a regra de multiplicação $A_{i} A_{j} \subseteq$ $A_{i+j \bmod 2,}$ é dita uma álgebra $\mathbb{Z}_{2}$ graduada ou uma superálgebra.

A soma em $A_{0}+A_{1}$ representa a soma direta de espaços vetoriais. As componentes $A_{0}, A_{1}$ são ditas componentes de graduação da superálgebra, em particular $A_{0}$ e $A_{1}$ são ditas a parte par e a parte ímpar de $A$ respectivamente. Note também que $A_{0}$ é uma subálgebra de $A$ e $A_{1}$ é um $A_{0}$-bimódulo.

\section{Exemplo 3.1}

1. Toda álgebra $A$ possui estrutura natural de superálgebra. $A_{0}=A e$ $A_{1}=0$.

2. $\mathbb{C}=\mathbb{R}+\mathbb{R} \cdot i$, álgebra dos números complexos é uma superálgebra sobre $\mathbb{R}$, com componentes $\mathbb{C}_{0}=\mathbb{R}, \mathbb{C}_{1}=\mathbb{R} \cdot i$.

3. $A=\mathbb{F} \dot{+} \mathbb{F} \cdot x, \quad x^{2}=0$, álgebra dos números duais é uma superálgebra, com componentes $A_{0}=\mathbb{F}, A_{1}=\mathbb{F} \cdot x$

4. $\mathbb{F}[u, \alpha]:=\mathbb{F}+\mathbb{F} \cdot u, \quad u^{2}=\alpha$, é uma superálgebra, em particular, quando $\alpha=1$ temos $\mathbb{F}[u, 1]:=\mathbb{F}[u]:=\mathbb{F}+\mathbb{F} \cdot u, \quad u^{2}=1$. 
5. $A[u]=A \dot{+} A \cdot u, \quad u^{2}=1, \mathrm{e}(\mathrm{a}+\mathrm{b} \cdot \mathrm{u})\left(\mathrm{a}^{\prime}+\mathrm{b}^{\prime} \cdot \mathrm{u}\right)=\left(\mathrm{aa}^{\prime}+\mathrm{bb}^{\prime}\right)+\left(\mathrm{ab}^{\prime}+\mathrm{ba}^{\prime}\right) \mathrm{u}$ é uma superálgebra com componentes $A_{0}=A, A_{1}=A \cdot u$.

6. Seja $V=V_{0}+V_{1}$ um superespaço vetorial, e seja $E=$ End $V$ a álgebra dos endomorfismos de $V$, então $E$ possui estrutura de superálgebra. Seja

$$
E_{i}=\left\{\psi \in E: \psi\left(V_{j}\right) \subseteq V_{i+j \bmod 2}, \quad j=0,1\right\}, \quad i=0,1 .
$$

$\operatorname{assim} E=E_{0} \dot{+} E_{1}$ e $E_{i} E_{j} \subseteq E_{i+j \bmod 2}$.

$\operatorname{Sejam~dim}_{\mathbb{F}} V=n<\infty$ e $n=m+k, \operatorname{dim}_{\mathbb{F}} V_{0}=m, \operatorname{dim}_{\mathbb{F}} V_{1}=k$. Fixando bases para $V_{0}, V_{1}$ obtemos uma base para $V$ e a partir desta obtemos um isomorfismo entre $E$ e $M_{m+k}(\mathbb{F})$ o qual induz uma estrutura de superálgebra em $M_{m+k}(\mathbb{F})$ com componentes de graduação dadas por:

$$
\left.\begin{array}{l}
\left(M_{m+k}(\mathbb{F})\right)_{0}=\left\{\left[\begin{array}{cc}
A & 0 \\
0 & D
\end{array}\right], \quad A \in M_{m}, D \in M_{k}\right\} \\
\left(M_{m+k}(\mathbb{F})\right)_{1}=\left\{\left[\begin{array}{ll}
0 & B \\
C & 0
\end{array}\right], \quad B \in M_{m \times k}, C \in M_{k \times m}\right.
\end{array}\right\}
$$

Definição 3.2 Definimos a superálgebra de matrizes $M(m \mid k)$, com componentes $(M(m \mid k))_{0}$ dada por (3.1), e $(M(m \mid k))_{1}$ dada por (3.2).

Seja $A$ uma superálgebra e $V \subseteq A$, dizemos que $V$ é um subsuperespaço de $A$ se $V=\left(V \cap A_{0}\right)+\left(V \cap A_{1}\right)$. Um sub-superespaço $V$ de $A$ o qual é uma subálgebra é dito uma subsuperálgebra de $A$. Um subsuperespaço $V$ de $A$ o qual é ideal de $A$ como álgebra será chamado ideal da superálgebra $A$.

Dadas $A, B$ superálgebras, um homomorfismo de superálgebras $\psi: A \longrightarrow B$ é um homomorfismo de álgebras que satisfaz $\psi\left(A_{i}\right) \subseteq B_{i}$ para $i=0,1$.

Definição 3.3 Seja $A=A_{0} \dot{+} A_{1}$ uma superálgebra e $f \in A_{0} \cup A_{1}$. Dizemos que $f$ tem paridade $i=0,1$ se $f \in A_{i}$. Vamos denotar a paridade de $f$ por $\vec{f}$. Também empregaremos a notaçẩo $\bar{a}=i$, sempre que $a_{i} \in A_{i}$. 


\subsection{Superálgebras Simples}

Uma superálgebra $A$ é dita simples, se $A^{2} \neq 0$ e $A$ não contém ideais próprios, (ideais graduados não nulos).

Teorema 3.4 Seja $A$ uma superálgebra simples então ou A é simples como álgebra ou $A \cong A_{0}[u]$, onde $A_{0}$ é uma álgebra simples.

Demonstração: $\quad$ Seja $A=A_{0} \dot{+} A_{1}$ uma superálgebra simples, e seja $I$ um ideal próprio de $A$ como álgebra, ( $I$ não é ideal graduado), sejam $\pi_{i}: A \longrightarrow A_{i}$ para $i=0,1$ as projeções canônicas de $A$. Sejam $I_{i}=A_{i} \cap I$ portanto $\operatorname{id}_{A}\left\langle I_{i}\right\rangle=\{0\}$ pois os $\operatorname{id}_{A}\left\langle I_{i}\right\rangle$ são ideais graduados de $A$ e $A$ é simples como superálgebra, assim temos que $I_{i}=\{0\}$ para $i=0,1$.

Seja $J=\pi_{0}(I)+\pi_{1}(I)$, logo $J$ é um ideal graduado de $A$ e $I \subseteq J$, pela simplicidade de $A$ temos $J=A$, portanto, $A_{i}=\pi_{i}(I)$ para $i=0,1$. Assim, para cada $a \in A_{i}$ existe um $j \in I$ tal que $\pi_{i}(j)=a$, mais ainda, o elemento $j \in I$ é o único com essa propriedade.

De fato, suponhamos que existem $j, j^{\prime} \in I$ tais que $\pi_{i}(j)=\pi_{i}\left(j^{\prime}\right)=a$, isto é $j=a+j_{1-i}$ e $j^{\prime}=a+j_{1-i}^{\prime}$ donde $j-j^{\prime}=j_{1-i}-j_{1-i}^{\prime} \in I_{1-i}=\{0\}$, portanto $j=j^{\prime}$. A existência e unicidade dos $j$ para cada $a_{i}$ permite-nos definir a seguinte função $u: A \longrightarrow A$ onde $u\left(a_{i}\right)=\pi_{1-i}(j)$ sendo $j$ o único elemento de $I$ que satisfaz $\pi_{i}(j)=a$, pela definição de $u$ temos que $u\left(A_{i}\right) \subseteq A_{1-i}$ e $u$ satisfaz as seguintes propriedades.

1. $u^{2}=\mathrm{id}$.

$2.1 \forall a, b \in A_{0} \cup A_{1}, \quad u(a b)=a u(b)=u(a) b$.

$2.2 \forall a, b \in A_{0}, \quad u(a) u(b)=u(u(a) b)=u(u(a b))=a b$.

portanto

$(a+u(b))\left(a^{\prime}+u\left(b^{\prime}\right)\right)=\left(a a^{\prime}+b b^{\prime}\right)+\left(a u\left(b^{\prime}\right)+u(b) a^{\prime}\right)=\left(a a^{\prime}+b b^{\prime}\right)+u\left(a b^{\prime}+b a^{\prime}\right)$ 
para todo $a, a^{\prime}, b, b^{\prime} \in A_{0}$. Assim $A$ é isomorfa a $A_{0}[u]$, com $A_{0}$ simples. Se $J$ é um ideal de $A_{0}$ então $J[u]$ é ideal graduado de $A_{0}[u]$.

Observação 3.5 Do teorema anterior temos que se $A$ é uma superálgebra simples na qual a parte par não é uma álgebra simples, então A é simples como álgebra.

Lema 3.6 Uma álgebra $A$ admite uma $\mathbb{Z}_{2}$-graduação não trivial se, e somente se, existe um automorfismo $\phi$ de $A$ tal que $\phi \neq \mathrm{id} e \phi^{2}=\mathrm{id}$, onde id representa o automorfismo identidade.

Demonstração: $\quad$ Seja $A=A_{0}+A_{1}$ uma superálgebra, definamos a função $\phi: A \longrightarrow A$ tal que $\phi\left(a_{0}+a_{1}\right)=a_{0}-a_{1}$, para todo $a_{0} \in A_{0}, a_{1} \in A_{1}$, assim $\phi$ é automorfismo de $A$, e satisfaz $\phi^{2}=\mathrm{id}$.

Seja $\phi$ automorfismo de $A$ tal que $\phi^{2}=i d \neq \phi$. Consideremos os autoespaços do operador $\phi$ associados com os autovalores $1,-1$, dados respectivamente por

$$
A_{0}=\{a \in A, \quad \phi(a)=a\}, \quad A_{1}=\{a \in A, \quad \phi(a)=-a\}
$$

Vejamos que $A_{0}, A_{1}$ é uma graduação de $A$. Sejam $a \in A_{i}, b \in A_{j}$, como $\phi$ é automorfismo, temos que $\phi(a b)=\phi(a) \phi(b)=(-1)^{i}(a)(-1)^{j}(b)=(-1)^{i+j}(a b)$. Portanto $A_{0}{ }^{2} \subseteq A_{0}, A_{1}{ }^{2} \subseteq A_{0}, A_{0} A_{1} \subseteq A_{1}, A_{1} A_{0} \subset A_{1}$ e assim $A$ é uma superálgebra.

Teorema 3.7 (C.T.C.Wall) Seja $A=A_{0}+A_{1}$ uma superálgebra associativa e simples de dimensão finita sobre um corpo $\mathbb{F}$ de característica diferente de 2 e algebricamente fechado, então $A$ é de alguns dos tipos $M_{n}[u]$ ou $M(k \mid m)$.

Demonstração: Pelo Teorema 3.4, temos somente dois casos a considerar, $A$ é simples como álgebra ou $A \cong B[u]$ para alguma álgebra simples $B$. Temos assim: 
1. Se $A$ não é simples como álgebra. Pelo Teorema 3.4 , temos que $A$ é isomorfa com $A_{0}[u]$, onde $A_{0}$ é uma álgebra simples, neste caso $A_{0} \cong$ $M_{n}(\mathbb{F})$, pois $A$ é associativa e de dimensão finita.

2. $A$ é simples como álgebra, podemos supor que $A \cong M_{n}(\mathbb{F})$.

Como $A$ é uma superálgebra, pelo Lema 3.6, existe um automorfismo $\phi$ de $A$ tal que $\phi^{2}=$ id $\neq \phi$, mas todo automorfismo em $M_{n}(\mathbb{F})$ é interno, (pelo Teorema de Noether-Skolem), portanto existe um $x \in A$ invertível tal que $\forall a \in A, \phi(a)=x^{-1} a x$, mas $\phi^{2}=$ id portanto $a=x^{-2} a x^{2}$. Assim temos que $a x^{2}=x^{2} a, \operatorname{logo} x^{2} \in Z(A)=Z\left(M_{n}(\mathbb{F})\right)=\mathbb{F}$, portanto existe um $0 \neq \alpha \in \mathbb{F}$ tal que $x^{2}=\alpha$, mas $\mathbb{F}$ é algebricamente fechado. Seja $y=x / \sqrt{\alpha}$.

Temos então que $y^{2}=1$ e invertível, assim $\phi(a)=y^{-1} a y$. Consideremos $A \cong M_{n}(\mathbb{F}) \cong \operatorname{End}_{\mathbb{F}} V$ onde $V$ é um espaço vetorial de dimensão $n$. Seja $y: V \longrightarrow V$, como $y^{2}=$ id então o espaço $V$ pode decompor-se como $V_{0}+V_{1}$, onde os espaços $V_{i}=\left\{a \in V: y(a)=(-1)^{i} a\right\}$ são os autoespaços associados com os autovalores $1,-1$ respectivamente. Seja $k=\operatorname{dim}_{\mathbb{F}} V_{0}$ e $m=\operatorname{dim}_{\mathbb{F}} V_{1} \operatorname{logo}$ End $V \cong M(m \mid k)$. Resta-nos demonstrar que $A=A_{0}+A_{1} \cong$ End $V$, como superálgebras, para isso vamos demonstrar que

$$
\begin{aligned}
& a \in A_{0} \text { se e somente se } a\left(V_{i}\right) \subseteq V_{i} \quad i=0,1 . \\
& a \in A_{1} \text { se e somente se } a\left(V_{i}\right) \subseteq V_{1-i} \quad i=0,1 .
\end{aligned}
$$

Seja $a \in A_{0}$, logo $\phi(a)=a=y^{-1} a y$, portanto $y a=a y$. Desse modo temos que se $v \in V_{i}$ então $y(v)=(-1)^{i}(v)$. Assim $y(a(v))=a y(v)=$ $a\left((-1)^{i} v\right)=(-1)^{i} a(v)$, portanto $a(v) \in V_{i}$.

Se $a \in A_{1}$ então $\phi(a)=-a=y^{-1} a y$ donde $y a=-a y$. Desse modo temos que se $v \in V_{i}$ então $y(v)=(-1)^{i} v$, portanto, $y(a(v))=-a y(v)=$ $-a\left((-1)^{i} v\right)=(-1)^{1-i} a(v)$, ou seja, $a(v) \in V_{1-i}$. 
Reciprocamente se $a\left(V_{i}\right) \subseteq V_{i}$ então para todo $v \in V_{i}$ temos $\phi(a)(v)=$ $y^{-1} a y(v)=(-1)^{i} y^{-1} a(v)$, como $a(v) \in V_{i}$ temos que $\phi(a)(v)=a(v)$ $\operatorname{logo} a \in A_{0}$, do mesmo modo prova-se $a\left(V_{1}\right) \subseteq V_{1-i}$ então $a \in A_{1}$.

\subsection{Superálgebra de Grassmann}

\subsection{1 Álgebra de Grassmann}

Seja $\Gamma$ uma álgebra asșociativa com 1 sobre $\mathbb{F}$ com característica de $\mathbb{F}$ diferente de 2 , gerada por 1 e pelos elementos $\left\{e_{1}, e_{2}, \ldots, e_{n}, \ldots\right\}$ tais que $e_{i}^{2}=0$ para todo $i$ e $e_{i} e_{j}=-e_{j} e_{i}$ para todo $i \neq j$, tal álgebra $\Gamma$ é chamada álgebra de Grassmann (álgebra do produto exterior).

Pela propriedade multiplicativa dos elementos $e_{i}$ temos que uma base para $\Gamma$ é determinada pelos produtos da forma $e_{i_{1}} e_{i_{2}} \ldots e_{i_{n}}$ onde $i_{1}<i_{2}<\cdots<i_{n}$. Assim se o conjunto gerador $\left\{e_{1}, \cdots, e_{n}, \cdots\right\}$ tem ordem finita $n$ então a dimensão de $\Gamma$ é $2^{n}$.

Vamos ver que $\Gamma$ possui estrutura de superálgebra, para isso, seja $\Gamma_{0}$ o espaço gerado por 1 e pelos produtos $e_{i_{1}} e_{i_{2}} \cdots e_{i_{2 n}}$ de comprimento par, e seja $\Gamma_{1}$ o espaço gerado pelos produtos $e_{i_{1}} e_{i_{2}} \cdots e_{i_{2 n+1}}$ de comprimento ímpar, assim é claro que $\Gamma=\Gamma_{0}+\Gamma_{1}$ e $\Gamma_{i} \Gamma_{j} \subseteq \Gamma_{i+j \bmod 2}$ portanto $\Gamma$ é uma superálgebra sobre $\mathbb{F}$, a qual chamaremos superálgebra de Grassmann gerada por $\left\{1, e_{1}, \cdots, e_{n}, \cdots\right\}$. No caso em que o conjunto gerador tenha ordem finita $n$, denotamos à álgebra por $\Gamma_{n}$ e por $\Gamma_{\infty}$ quando o conjunto gerador é de ordem infinita.

Dada a anticomutatividade nas variáveis $\left\{e_{1}, e_{2} \cdots e_{n}, \cdots\right\}$ e as propriedades do grupo simétrico $\operatorname{Sym}(n)$ temos o seguinte:

1. Se $f \in \Gamma_{0}$ e $g \in \Gamma_{0} \cup \Gamma_{1}$ então $f g=g f$

2. Se $f, g \in \Gamma_{1}$ então $f g=-g f$ 
Do anterior podemos garantir que dados $f, g \in \Gamma_{0} \cup \Gamma_{1}$ então

$$
f g-(-1)^{\bar{f} \bar{g}} g f=0
$$

A Identidade dada em (3.3) é chamada identidade supercomutativa.

Definição 3.8 Seja $A=A_{0}+A_{1}$ uma superálgebra e consideremos o produto tensorial de álgebras $\Gamma \otimes A$. É fácil verificar que o subespaço $\Gamma(A)=\Gamma_{0} \otimes$ $A_{0}+\Gamma_{1} \otimes A_{1}$ é uma subálgebra de $\Gamma \otimes A$, a qual é chamada de envolvente de Grassmann de $A$.

Definição 3.9 Seja $\mathfrak{M}$ uma variedade de álgebras homogênea. Uma Msuperálgebra é uma superálgebra $A$ tal que $\Gamma(A) \in \mathfrak{M}$.

Como continuação vamos ver alguns exemplos de superálgebras onde as identidades são obtidas a partir da álgebra envolvente de Grassmann. Somente vamos fazer a prova detalhada no caso das superálgebras comutativas e de Jordan, os outros casos são desenvolvidos de maneira análoga. Também veremos a importância que as identidades que definem a álgebra sejam multilineares.

\section{Exemplo 3.10}

1. Uma superálgebra $A=A_{0} \dot{+} A_{1}$ é comutativa se $\Gamma(A)$ é comutativa, equivalentemente, $A$ é comutativa se satisfaz a superidentidade $a_{i} b_{j}=$ $(-1)^{i j} b_{j} a_{i}$ para $a_{i} \in A_{i}, b_{j} \in A_{j}$.

De fato, $\Gamma(A)$ é comutativa se, e somente se, para todo $f_{i}, g_{j} \in \Gamma_{0} \cup \Gamma_{1}$ e para todo $a_{i}, a_{j} \in A_{0} \cup A_{1}$ temos

$$
\left(f_{i} \otimes a_{i}\right)\left(g_{j} \otimes a_{j}\right)-\left(g_{j} \otimes a_{j}\right)\left(f_{i} \otimes a_{i}\right)=0
$$


Mas, pela Propriedade 3.3 , para $\Gamma$ temos

$$
\begin{aligned}
0 & =\left(f_{i} \otimes a_{i}\right)\left(g_{j} \otimes a_{j}\right)-\left(g_{j} \otimes a_{j}\right)\left(f_{i} \otimes a_{i}\right) \\
& =f_{i} g_{j} \otimes a_{i} a_{j}-g_{j} f_{i} \otimes a_{j} a_{i} \\
& =f_{i} g_{j}\left(a_{i} a_{j}-(-1)^{i j} a_{j} a_{i}\right)
\end{aligned}
$$

e como $f_{i} \neq 0, g_{j} \neq 0$ e para $i \neq j f_{i} g_{j} \neq 0$, então da igualdade em (3.4), temos que $a_{i} b_{j}-(-1)^{i j} b_{j} a_{i}=0$

Do anterior e da Identidade 3.3, é claro a seguinte Proposição:

Proposição 3.11 A álgebra de Grassmann é uma superálgebra comutativa.

2. Lembramos que $L$ é uma álgebra de Lie com multiplicação $[a, b]$ se para todo $a, b, c \in L$ cumpre-se

$$
\begin{array}{r}
{[a, b]+[b, a]=0} \\
{[[a, b], c]+[[b, c], a]+[[c, a], b]=0}
\end{array}
$$

Uma superálgebra $L=L_{0} \dot{+} L_{1}$ é uma superálgebra de Lie, se satisfaz as superidentidades

$$
\begin{gathered}
{[a, b]=(-1)^{\bar{a} \bar{b}}[b, a]} \\
(-1)^{\bar{a} \bar{c}}[[a, b], c]+(-1)^{\bar{a} \bar{b}}[[b, c], a]+(-1)^{\bar{b} \bar{c}}[[c, a], b]=0
\end{gathered}
$$

3. Seja $A$ uma álgebra alternativa. Logo $(a, a, b)=(b, a, a)=0$ para todo $a, b \in A, \mathrm{e}(a, b, c)$ representa a função associador. Uma superálgebra $A$ é alternativa se para todo $a, b, c \in A_{0} \cup A_{1}$ e $x_{0} \in A_{0}$, se satisfazem as seguintes superidentidades

$$
\begin{aligned}
(a, b, c)+(-1)^{\bar{b} \bar{c}}(a, c, b) & =0 \\
(a, b, c)+(-1)^{\bar{a} \bar{b}}(b, a, c) & =0 \\
\left(x_{0}, x_{0}, b\right) & =0
\end{aligned}
$$


4. Uma superálgebra $J$ é uma superálgebra de Jordan, se para todo $a_{i}, a_{j}, a_{k}$, $a_{l} \in J_{0} \cup J_{1}$ se satisfazem as superidentidades

$$
\begin{aligned}
0 & =a_{i} b_{j}-(-1)^{i j} b_{j} a_{i} \\
0 & =(-1)^{l(i+k)}\left(a_{i} a_{j}, a_{k}, a_{l}\right)+(-1)^{i(j+k)}\left(a_{j} a_{l}, a_{k}, a_{i}\right) \\
& +(-1)^{j(l+k)}\left(a_{l} a_{i}, a_{k}, a_{j}\right)
\end{aligned}
$$

$J$ é uma superálgebra de Jordan se $\Gamma(J)$ é de Jordan, isto é, $\Gamma(J)$ é comutativa e satisfas a identidade de Jordan, a qual em corpos de característica diferente de três é equivalente com a identidade linearizada de Jordan $(a d, b, c)+(a c, b, d)+(d c, b, a)=0$. Como vimos no exemplo 1 , a comutatividade equivale com a identidade $a_{i} b_{j}-(-1)^{i j} b_{j} a_{i}=0$. Vejamos agora que (3.13), é induzida pela identidade linearizada de Jordan.

Sejam $f_{i}, f_{j}, f_{k}, f_{l} \in \Gamma_{)} \cup \Gamma_{1}$ e $a_{i}, a_{j}, a_{k}, a_{l} \in J_{0} \cup J_{1}$ elementos não nulos. Então da linearização completa de Jordan em $\Gamma(J)$ temos:

$$
\begin{aligned}
0= & \left(\left(f_{i} \otimes a_{i}\right)\left(f_{j} \otimes a_{j}\right), f_{k} \otimes a_{k}, f_{l} \otimes a_{l}\right)+\left(\left(f_{i} \otimes a_{i}\right)\left(f_{l} \otimes a_{l}\right), f_{k} \otimes a_{k}, f_{j} \otimes a_{j}\right) \\
& +\left(\left(f_{j} \otimes a_{j}\right)\left(f_{l} \otimes a_{l}\right), f_{k} \otimes a_{k}, f_{i} \otimes a_{i}\right) \\
= & f_{i} f_{j} f_{k} f_{l} \otimes\left(a_{i} a_{j}, a_{k}, a_{l}\right)+f_{i} f_{l} f_{k} f_{j} \otimes\left(a_{i} a_{l}, a_{k}, a_{j}\right)+f_{j} f_{l} f_{k} f_{i} \otimes\left(a_{j} a_{l}, a_{k}, a_{i}\right) \\
= & f_{i} f_{j} f_{k} f_{l} \otimes\left((-1)^{l(i+k)}\left(a_{i} a_{j}, a_{k}, a_{l}\right)+(-1)^{i(j+k)}\left(a_{j} a_{l}, a_{k}, a_{i}\right)\right. \\
& \left.+(-1)^{j(l+k)}\left(a_{l} a_{i}, a_{k}, a_{j}\right)\right)
\end{aligned}
$$

para todo $f_{i}, f_{j}, f_{k}, f_{l} \in \Gamma_{0} \cup \Gamma_{1}$ e para todo $a_{i}, a_{j}, a_{k}, a_{l} \in J_{0} \cup J_{1}$ onde $\bar{f}_{s}=\bar{a}_{s}=s$, para $s \in\{i, j, k, l\}$ e $s=0,1$.

Portanto

$0=(-1)^{l(i+k)}\left(a_{i} a_{j}, a_{k}, a_{l}\right)+(-1)^{i(j+k)}\left(a_{j} a_{l}, a_{k}, a_{i}\right)+(-1)^{j(l+k)}\left(a_{l} a_{i}, a_{k}, a_{j}\right)$ 
Observação 3.12 Note que se $\mathfrak{M}$ é uma variedade e $A=A_{0}+A_{1}$ é uma $\mathfrak{M}$-superálgebra, então $A_{0}$ pertence à variedade $\mathfrak{M}$.

Como continuação, vamos verificar que toda álgebra $A$ numa variedade $\mathfrak{M}$ definida por un conjunto de identidades multilineares induz uma estrutura de superálgebra via a superálgebra de Grassmann.

Seja $A$ uma álgebra sobre o corpo $\mathbb{F}$ e $\Gamma$ a superálgebra de Grassmann. Definimos $A_{\Gamma}=\Gamma \otimes A$. Então $A_{\Gamma}$ é uma álgebra, mais ainda é uma superálgebra com componentes $\left(A_{\Gamma}\right)_{0}=\Gamma_{0} \otimes A,\left(A_{\Gamma}\right)_{1}=\Gamma_{1} \otimes A$.

Proposição 3.13 Seja $\mathfrak{M}$ uma variedade definida por um conjunto de identidades multi-lineares e $A \in \mathfrak{M}$. Enião $A_{\Gamma}$ é uma $\mathfrak{M}$-superálgebra.

Demonstração: $\quad$ Para verificar que $A_{\Gamma}$ é uma M-superálgebra só precisamos provar que $\Gamma\left(A_{\Gamma}\right) \in \mathfrak{M}$. Temos que

$$
\begin{aligned}
& \Gamma\left(A_{\Gamma}\right)=\left(\Gamma \otimes\left(\Gamma_{0} \otimes A \dot{+} \Gamma_{1} \otimes A\right)\right)_{0}=\Gamma_{0} \otimes\left(\Gamma_{0} \otimes A\right) \dot{+} \Gamma_{1} \otimes\left(\Gamma_{1} \otimes A\right)= \\
& \Gamma_{0} \otimes \Gamma_{0} \otimes A \dot{+} \Gamma_{1} \otimes \Gamma_{1} \otimes A=\left(\Gamma_{0} \otimes \Gamma_{0} \dot{+} \Gamma_{1} \otimes \Gamma_{1}\right) \otimes A=\Gamma(\Gamma) \otimes A .
\end{aligned}
$$

mas $\Gamma(\Gamma)$ é comutativa e associativa, assim, pela Proposição 1.18 , temos que toda identiclade em $A$ se satisfaz em $A_{\Gamma}$. Portanto, $A_{\Gamma}$ é uma $\mathfrak{M}$-superálgebra.

A Proposição anterior nos garante que toda álgebra numa variedade $\mathfrak{M}$ definida por um conjunto de identidades multi-lineares, pode ser imersa numa M-superálgebra $A_{\Gamma}$ por meio de uma extensão do corpo dos escalares $\mathbb{F}$ ao domínio dos superescalares $\Gamma$.

\subsection{Outros exemplos de superálgebras}

Vamos apresentar a seguir alguns exemplos de superálgebras que não são obtidos a partir da envolvente de Grasmman $\Gamma(A)$ e cujas construções são mais técnicas. 


\subsubsection{Superálgebra de Clifford}

O produto tensorial $A \otimes B$ de superespaços $A, B$ tem uma estrutura natural de superespaço, $(A \otimes B)_{0}=A_{0} \otimes B_{0}+A_{1} \otimes B_{1}$ e $(A \otimes B)_{1}=A_{0} \otimes B_{1}+A_{1} \otimes B_{0}$, mas $A \otimes B$ como superálgebra tem duas estruturas naturais de superálgebra, que são a saber, a dada pelo produto tensorial $(a \otimes b)\left(a^{\prime} \otimes b^{\prime}\right)=a a^{\prime} \otimes b b^{\prime}$, e a dada pelo produto tensorial torcido $\left(a \widetilde{\otimes} b_{i}\right)\left(a_{j} \widetilde{\otimes} b^{\prime}\right)=(-1)^{i j}\left(a a_{j} \widetilde{\otimes} b_{i}^{\prime}\right)$ para $a \in A, a_{j} \in A_{j}, b^{\prime} \in B, b_{i} \in B_{i}$.

Pela definição do produto tensorial torcido é claro que para $a \in A, c, b^{\prime} \in$ $B, a_{j} \in A_{j}$ que $(a \tilde{\otimes} c)\left(a_{j} \widetilde{\otimes} b^{\prime}\right)=\left(a a_{j} \widetilde{\otimes} c_{0} b^{\prime}\right)+(-1)^{j}\left(a a_{j} \widetilde{\otimes} c_{1} b^{\prime}\right) a \in A, c, b^{\prime} \in B$, $a_{j} \in A_{j}$. Desta relação se deduz facilmente o seguinte Lema.

Lema 3.14 Se $A$ e $B$ são superálgebras associativas, então $A \widetilde{\otimes} B$ também é.

Seja $\alpha \in \mathbb{F}$, definimos a superálgebra $A(\alpha)=\mathbb{F}+\mathbb{F} \cdot u$ onde $u^{2}=\alpha$, e as componentes $A(\alpha)_{0}=\mathbb{F}, A(\alpha)_{1}=\mathbb{F} \cdot u$. Sejam $\alpha_{1}, \alpha_{2}, \ldots, \alpha_{n} \in \mathbb{F}$, definimos a superálgebra $\operatorname{Cl}\left(\alpha_{1}, \ldots, \alpha_{n}\right):=\widetilde{\otimes}_{i=1}^{n} A\left(\alpha_{i}\right)$, onde $\widetilde{\otimes}$ denota o produto tensorial torcido.

Se $\mathbb{F}$ é um corpo algebricamente fechado, podemos supor que todos os $\alpha_{i} \neq 0$ são 1 , no caso em que todos sejam 1 , escrevemos $\mathrm{Cl}_{n}=\mathrm{Cl}(1, \ldots, 1)$.

Denotamos por $u_{i} \in \mathrm{Cl}_{n}$ o elemento $1 \widetilde{\otimes} \cdots \widetilde{\otimes} u \widetilde{\otimes} \cdots \widetilde{\otimes} 1$, onde $u$ está na posição $i$ e $u^{2}=1$. Sejam $u_{i}, u_{j} \in \mathrm{Cl}_{n}$ temos pela definição do produto torcido $u_{i} u_{j}=-u_{j} u_{i}$. A álgebra $\mathrm{Cl}\left(\alpha_{1}, \ldots, \alpha_{n}\right)$ é uma superálgebra associativa chamada álgebra de Clifford.

Observemos que, como $\operatorname{dim} A \otimes B=\operatorname{dim} A \cdot \operatorname{dim} B$, resulta claro que $\operatorname{dim} \mathrm{Cl}_{n}=2^{n}$.

Um caso especial da álgebra de Clifford acontece quando os $\alpha_{i}$ são todos nulos, nesse caso, $\mathrm{Cl}_{n}=\Gamma_{n}$ é a superálgebra de Grassmann em $n$ variáveis. 


\subsubsection{Superálgebra $W_{m}$}

Seja $W_{1}=\operatorname{alg}\langle x, y: x y-y x=1\rangle=T(\{x, y\}) /$ id $\langle x y-y x-1\rangle$.

$W_{1}$ é dita a álgebra de Weyl.

Definimos $W_{m}=W_{1}^{\otimes m}=W_{m}=T\left(\left\{x_{1}, \ldots, x_{m} ; y_{1}, \ldots, y_{m}\right\}\right) / I$ onde $I=$ id $\left\langle x_{i} x_{j}-x_{j} x_{i}, y_{i} y_{j}-y_{j} y_{i}, x_{i} y_{j}-y_{j} x_{i}-\delta_{i j} \cdot 1\right\rangle$.

Proposição 3.15 Seja A uma álgebra associativa com 1 sobre um corpo de característica zero, suponhamos que existem em $A$ elementos $x, y$ tais que $x y-y x=1$, então, os elementos $x^{i} y^{j}$ são linearmente independentes em $A$ $e \operatorname{alg}_{A}\langle x, y\rangle \cong W_{1}$.

Demonstração: Ver [19].

Observação $3.16 W_{m}$ tem estrutura natural de superálgebra, basta considerar $\overline{x_{i}}=\overline{y_{i}}=1$ para $i=1,2, \ldots, m . x_{i}, y_{i}$ denotam as variáveis livres na álgebra livre $T\left(\left\{x_{1}, \ldots, x_{m} ; y_{1}, \ldots, y_{m}\right\}\right)$

\subsubsection{Superálgebras de colchetes}

Seja $A$ uma álgebra associativa, comutativa com 1 , e $\{\}:, A \times A \longrightarrow A$ uma aplicação bilinear anti-simétrica, a qual chamamos de colchete. Consideremos a soma de espaços vetoriais $J(A)=A \dot{+} A x$, munida da seguinte multiplicação. Para $a, b \in A$, a multiplicação em $A$ vai ser representada por $a b$ e a multiplicação em $J(A)$ por um ponto $\cdot$ Assim,

$$
a \cdot b=a b, a \cdot b x=a b \cdot x, a x \cdot b=a b \cdot x, a x \cdot b x=\{a, b\}
$$

É evidente que $J(A)$ é uma superálgebra comutativa. $J(A)$ é dita superálgebra dobrada de Kantor. O processo descrito é chamado processo de dobramento de Kantor.

Definição 3.17 Seja A uma álgebra com as propriedades acima. O colchete $\{$,$\} é dito de Jordan se a superálgebra J(A)$ é de Jordan. 
O seguinte resultado demonstrado por McCrimmon em [9] e [10], nos permite ter uma caracterização do colchete de Jordan em termos do operador colchete.

Proposição 3.18 Seja A uma álgebra comutativa, associativa, com 1 e que possui um colchete antisimétrico $\{$,$\} . O colchete \{$,$\} é de Jordan se, e$ somente se, satisfaz as seguintes identidades.

$$
\begin{aligned}
\{a b, c\} & =a\{b, c\}+\{a, c\} b-a b\{c, 1\} \\
\{\{a, b\}, c\} & =-\{\{b, c\}, a\}-\{\{c, a\}, b\} \\
& =+\{a, b\}\{c, 1\}+\{b, c\}\{a, 1\}+\{c, a\}\{b, 1\}
\end{aligned}
$$

Definição 3.19 Seja A uma álgebra. Uma aplicação $D: A \longrightarrow A$ é dita uma derivação em $A$, se satisfaz $D(x y)=D(x) y+x D(y)$.

Consideremos uma álgebra $A$ com as propriedades descriptas na Proposição anterior. Definimos em $A$ a aplicação $D: A \longrightarrow A$ tal que $D(a):=$ $\{a, 1\}$.

Proposição 3.20 Seja A uma álgebra comutativa, associativa com 1 e que possui um produto colchete de Jordan. Então D é uma derivação de A.

Um colchete de Jordan sobre $A$ diz-se do tipo Poisson se $D(a)=0, \forall a \in$ A. Neste caso temos:

1. $\langle A,\{\},+$,$\rangle é uma álgebra de Lie.$

2. $\{a b, c\}=a\{b, c\}+\{a, c\} b \quad$ (A identidade de Leibniz).

Um colchete de Jordan diz-se do tipo vetorial se, $\{a, b\}=D(a) b-a D(b)$.

O Processo de Dobramento de Kantor pode ser estendido ao caso em que $A=A_{0} \dot{+} A_{1}$ é uma superálgebra associativa, comutativa com 1 e que possui um produto superanti-simétrico $\{$,$\} supercolchetes. Dada a graduação de$ 
$A$, temos que a graduação em $J\left(A_{0} \dot{+} A_{1}\right)$ é dada por $J\left(A_{0} \dot{+} A_{1}\right)_{0}=A_{0} \dot{+} A_{1} x$ e $J\left(A_{0} \dot{+} A_{1}\right)_{1}=A_{1} \dot{+} A_{0} x$.

Dados $a, b \in A_{0} \cup A_{1}$ representamos por $a b$ a multiplicação em $A$. Assim a multiplicação em $J\left(A_{0}+A_{1}\right)$ é definida por.

$$
a \cdot b=a b, a \cdot b x=(a b) x, a x \cdot b=(-1)^{\bar{b}}(a b) x, a x \cdot b x=(-1)^{\bar{b}}\{a, b\}
$$

Definição 3.21 Um supercolchete é dito de Jordan se a superálgebra $J\left(A_{0} \dot{+}\right.$ $\left.A_{1}\right)$ é uma superálgebra de Jordan.

A seguinte Proposição é uma extensão natural da Proposição 3.18, uma demonstração pode se encontrar em [9] e [10].

Proposição 3.22 Seja A uma superálgebra associativa, comutativa, com 1 e com um supercolchete $\{$,$\} . Então, \{$,$\} é um colchete de Jordan se, e$ somente se, satisfaz as seguintes identidades:

$$
\begin{aligned}
& \{a, b\}=-(-1)^{\bar{a} \bar{b}}\{b, a\} \\
& \{a, b c\}=\{a, b\} c+(-1)^{\bar{a} \bar{b}} b\{a, c\}-D(a) b c \\
& \{\{a, b\}, c\}+(-1)^{\bar{a}(\bar{b}+\bar{c})}\{\{b, c\}, a\}+(-1)^{\bar{c}(\bar{a}+\bar{b})}\{\{c, a\}, b\} \\
& =-\{a, b\} D(c)+(-1)^{\bar{a}(\bar{b}+\bar{c})}\{b, c\} D(a)+(-1)^{\bar{c}(\bar{a}+\bar{b})}\{c, a\} D(b) \\
& \{\{a, a\}, a\}=-\{a, a\} D(a)
\end{aligned}
$$

A Identidade (3.20), só é necessária no caso de corpos de característica três As definições no caso de colchetes de Jordan para superálgebras cle supercolchetes de tipo vetorial e de Poisson são as mesmas. 


\section{Capítulo 4}

\section{SUPERÁLGEBRAS DE JORDAN}

O objetivo deste Capítulo é apresentar os teoremas de classificação das superálgebras de Jordan simples de dimensão finita sobre um corpo algebricamente fechado.

Lembramos do Capítulo anterior que uma superálgebra $J$ é uma superálgebra de Jordan, se sua envolvente de Grassmann $\Gamma(J)$ é de Jordan, equivalentemente, se $J=J_{0} \dot{+} J_{1}$ satisfaz as identidades graduadas

$$
\begin{array}{r}
0=a_{i} b_{j}-(-1)^{i j} b_{j} a_{i} \quad \forall a_{s} \in J_{0} \cup J_{1}, \quad s=i, j ; \quad i, j=0,1 . \\
0=(-1)^{l(i+k)}\left(a_{i} a_{j}, a_{k}, a_{l}\right)+(-1)^{i(j+k)}\left(a_{j} a_{l}, a_{k}, a_{i}\right)+(-1)^{j(l+k)}\left(a_{l} a_{i}, a_{k}, a_{j}\right)
\end{array}
$$

Daqui em diante, dada uma superálgebra de Jordan $J=J_{0} \dot{+} J_{1}$, vamos denotar a parte par de $J, J_{0}$ por $A$, e a parte ímpar de $J, J_{1}$ por $M$. A multiplicação em $M$ vai ser representada por [, ]. 


\subsection{Em corpos de característica zero}

Vamos agora estudar as superálgebras de Jordan de dimensão finita sobre corpos de característica zero. Vamos ver que neste caso existem exemplos de superálgebras de Jordan similares com as álgebras de Jordan de forma bilinear e as álgebras de Jordan de tipo hermitiano apresentadas no Capítulo 2, além de outras, mas não existe uma superálgebra de Jordan do tipo Albert.

\section{1.) Superálgebras especiais de Jordan}

Seja $A=A_{0}+A_{1}$ uma superálgebra associativa e consideremos o produto supersimétrico $a \circ_{s} b=a b+(-1)^{\bar{a} \bar{b}} b a$, onde $a b$ é o produto em $A$. Definimos $A^{+s}=\left\langle A, \circ_{s}\right\rangle$.

Proposição 4.1 A superálgebra $A^{+s}$ é uma superálgebra de Jordan.

Demonstração: Para provar que $A^{+s}$ é uma superálgebra de Jordan, só temos que demonstrar que $\Gamma\left(A^{+s}\right)$ é de Jordan. Para isso, verificamos que $\Gamma\left(A^{+s}\right)=\Gamma(A)^{+}$. Note que os elementos são os mesmos, assim, só precisamos mostrar que as multiplicações são iguais.

Sejam $a, b \in A_{0} \cup A_{1}$ e $f, g \in \Gamma_{0} \cup \Gamma_{1}$ tais que $\bar{a}=\bar{f}, \bar{b}=\bar{g}$.

$$
\begin{aligned}
& \overbrace{(f \otimes a)(g \otimes b)}^{\in \Gamma\left(A^{+s}\right)}=f g \otimes\left(a \circ_{s} b\right)=f g \otimes\left(a b+(-1)^{\bar{a} \bar{b}} b a\right)= \\
& f g \otimes a b+f g \otimes(-1)^{\bar{a} \bar{b}} b a=f g \otimes a b+(-1)^{\bar{f} \bar{g}} f g \otimes b a \\
& f g \otimes a b+g f \otimes b a=\underbrace{(f \otimes a) \circ(g \otimes b)}_{\in \Gamma(A)^{+}}
\end{aligned}
$$

Daqui, podemos garantir que $\Gamma\left(A^{+s}\right)$ é uma álgebra especial de Jordan, e que a superálgebra $A^{+s}$ é uma superálgebra de Jordan.

Definição 4.2 Uma superálgebra de Jordan $J$ é dita especial se existe uma superálgebra associativa $A$ tal que $J$ pode ser imersa $\mathrm{em} A^{\text {t. }}$. 
Uma superálgebra de Jordan $J$ que não é especial, é dita uma superálgebra de Jordan excepcional.

Temos pelo Teorema 3.7, que sobre corpos algebricamente fechados de característica diferente de 2 , as únicas superálgebras associativas simples estão dadas por $M(m \mid k)$ e $M_{n}[u]$. Temos assim as superálgebras de Jordan especiais $M(m \mid k)^{+s}$ e $M_{n}[u]^{+s}$. No primeiro caso, temos a superálgebra $J=M(m \mid k)^{+s}$ com graduação dada por $A=M_{m} \oplus M_{k}$ e $M=M_{m \times k} \oplus M_{k \times m}$. A estrutura de $A$-bimódulo $M$, é dada pelos produtos $(a+b) \cdot c=\frac{1}{2}(a c+$ $c b),(a+b) \cdot d=\frac{1}{2}(d a+b c) \operatorname{com} a \in M_{m}, b \in M_{k}, c \in M_{m \times k}, d \in M_{k \times m}$. No segundo caso, $J=M_{n}[u]^{+s}$ com graduação dada por $A=M_{n}(\mathbb{F}), M=\overline{M_{n}(\mathbb{F})}$, $\operatorname{com} \overline{M_{n}(\mathbb{F})}$ copia isomorfa de $M_{n}(\mathbb{F})$. A estrutura de $A$-bimódulo $M$, é dada pelo produto $a \cdot \bar{b}=\frac{1}{2} \overline{(a b+b a)}, a, b \in M_{n}(\mathbb{F})$.

\section{2.) Superálgebras de Jordan do tipo Hermitiano}

Definição 4.3 Seja $A=A_{0}+A_{1}$ uma superálgebra. Uma superinvolução em. A é uma aplicação (*) tal que para todo $a, b \in A_{0} \cup A_{1},(a)^{* *}=a e$ $(a b)^{*}=(-1)^{\bar{a} \bar{b}} b^{*} a^{*}$.

Seja $A=A_{0} \dot{+} A_{1}$ uma superálgebra associativa e consideremos $*: A \mapsto A$ uma superinvolução em $A$. Seja $H(A, *)=\left\{a \in A_{0} \cup A_{1} / a=a^{*}\right\}$ o conjunto dos elementos $*$-simétricos. $H(A, *)$ é uma subsuperálgebra da superálgebra $A^{+s}$

Proposição $4.4 H(A, *)$ é uma superálgebra especial de Jordan.

Demonstração: $\quad$ Seja $\Gamma_{*}: \Gamma(A) \longrightarrow \Gamma(A)$, tal que $(f \otimes a)^{\Gamma_{*}}=f \otimes a^{*}$. Então $\Gamma_{*}$ é uma involução. De fato, sejam $f \otimes a, g \otimes b \in \Gamma(J)$, assim,

i). $\quad \Gamma_{*}((f \otimes a)(g \otimes b))=\Gamma_{*}(f g \otimes a b)=f g \otimes(a b)^{*}=(-1)^{\bar{f} \bar{g}} g f \otimes(-1)^{\bar{a} \bar{b}} b^{*} a^{*}$ $=\left(g \otimes b^{*}\right)\left(f \otimes a^{*}\right)=\Gamma_{*}(g \otimes b) \Gamma_{*}(f \otimes a)$

ii). $\quad \Gamma_{*}\left(\Gamma_{*}(f \otimes a)\right)=\Gamma_{*}\left(f \otimes a^{*}\right)=f \otimes a^{* *}=f \otimes a$ 
logo é involução.

Afirmação $H\left(\Gamma(A), \Gamma_{*}\right)=\Gamma(H(A, *))$

De fato, como conjuntos temos que

$$
\begin{aligned}
\Gamma(H(A, *)) & =\left\{f \otimes a: f \in \Gamma_{0} \cup \Gamma_{1}, a \in A_{0} \cup A_{1}, a^{*}=a\right\} \\
& =\left\{f \otimes a: f \in \Gamma_{0} \cup \Gamma_{1}, a \in A_{0} \cup A_{1}, f \otimes a^{*}=f \otimes a\right\} \\
& =\left\{f \otimes a: f \in \Gamma_{0} \cup \Gamma_{1}, a \in A_{0} \cup A_{1}, \Gamma_{*}(f \otimes a)=f \otimes a\right\} \\
& =H\left(\Gamma(A), \Gamma_{*}\right)
\end{aligned}
$$

As multiplicações num e noutro caso estão dadas por $(f \otimes a)(g \otimes b)=(f g \otimes a b)$.

A seguinte Proposição foi demonstrada por Gómez Ambroci em [7].

Proposição 4.5 Se A é uma superálgebra associativa com superinvolução *, tal que $(A, *)$ é*-simples, então $H(A, *)$ é simples.

Alguns casos particulares das superálgebras de Jordan do tipo hermitiano, são a superálgebra ortosimplética e a superálgebra de supertransposição.

\section{1) Superálgebra de Jordan ortosimplética Osp (m|2k)}

Seja $V=V_{0}+V_{1}$ um superespaço vetorial de dimensão finita $m+2 k$ onde $\operatorname{dim} V_{0}=m \operatorname{e~} \operatorname{dim} V_{1}=2 k$. Nós temos que $E=$ End $V \cong M(m \mid 2 k)$.

Consideremos a aplicação Osp : $M(m \mid 2 k) \longrightarrow M(m \mid 2 k)$ tal que:

$$
\left[\begin{array}{ll}
A_{m \times m} & B_{m \times 2 k} \\
C_{2 k \times m} & D_{2 k \times 2 k}
\end{array}\right]^{\text {Osp }}=\left[\begin{array}{ll}
E_{m} & 0 \\
0 & S_{2 k}
\end{array}\right]^{-1}\left[\begin{array}{ll}
A & -B \\
C & D
\end{array}\right]^{t}\left[\begin{array}{ll}
E_{m} & 0 \\
0 & S_{2 k}
\end{array}\right]
$$

onde $t$ denota a transposição usual de matrizes, e $S=\left[\begin{array}{cc}0 & 1_{k} \\ -1_{k} & 0\end{array}\right]$

Osp é uma superinvolução na superálgebra $M(m \mid 2 k)$, chamada de superinvolução ortosimplética.

Das Proposições 4.4 e 4.5 , temos o seguinte resultado 
Corolário 4.6 Seja $M(m \mid 2 k)$ uma superálgebra com superinvolução ortosimplética Osp. Então $H(M(m \mid 2 k)$, Osp) é uma superálgebra de Jordan especial simples.

Daqui em diante vamos denotar a superálgebra $H(M(m \mid 2 k)$, Osp $)$ por $\operatorname{Osp}(m \mid 2 k)$.

\section{2.) Superálgebra de Jordan $\operatorname{trp}(\mathbf{n} \mid \mathbf{n})$}

Consideremos a superálgebra associativa $M_{n}(\mathbb{F})$ e, definamos a aplicação trp $: M_{n}(\mathbb{F}) \longrightarrow M_{n}(\mathbb{F})$ tal que

$$
\left[\begin{array}{ll}
A & B \\
C & D
\end{array}\right]^{\text {trp }}=\left[\begin{array}{ll}
D^{t} & -B^{t} \\
C^{t} & A^{t}
\end{array}\right]
$$

Onde $t$ denota a transposição usual de matrizes, trp é uma superinvolução em $M_{n}(\mathbb{F})$ chamada a superinvolução transposição. Das Proposições 4.4 e 4.5 , temos o seguinte resultado

Corolário 4.7 Seja $M_{n}(\mathbb{F})=M(n \mid n) n>1$, com superinvolução supertransposição trp. Então $H(M(n \mid n)$, trp) é uma superálgebra de Jordan especial simples.

Daqui em diante vamos denotar a superálgebra $H(M(n \mid n), \operatorname{trp})$ por $\operatorname{trp}(n \mid n)$. A graduação de $\operatorname{trp}(n \mid n)$ é dada por $A=M_{n}(\mathbb{F}), M=S_{n}(\mathbb{F})+H_{n}(\mathbb{F})$, onde $S_{n}(\mathbb{F})$ representa o conjunto de matrizes anti-simétricas de ordem $n$.

\section{Superálgebra de superforma bilinear $\mathrm{J}(\mathrm{V}, \mathrm{f})$}

Definição 4.8 Sejam $V=V_{0}+V_{1}$ um superespaço vetorial e $f: V \times V \longrightarrow \mathbb{F}$ uma aplicação bilinear. Dizemos que $f$ é uma forma bilinear supersimétrica se $f\left(V_{i}, V_{j}\right)=0$ para $i \neq j$, e $f\left(v_{i}, w_{i}\right)=(-1)^{i} f\left(w_{i}, v_{i}\right) \forall v_{i}, w_{i} \in V_{i}, i=0,1$.

Isto é, f é uma forma bilinear supersimétrica se: 
1. $f\left(V_{0}, V_{1}\right)=f\left(V_{1}, V_{0}\right)=0$.

2. $f$ é simétrica em $V_{0}$.

3. $f$ é antisimétrica em $V_{1}$.

Lembremos que o produto tensorial torcido para duas superálgebras $A, B$ é dado pela multiplicação $\left(a \widetilde{\otimes} b_{i}\right)\left(a_{j}^{\prime} \widetilde{\otimes} b^{\prime}\right)=(-1)^{i j}\left(a a_{j}^{\prime} \widetilde{\otimes} b_{i} b^{\prime}\right), a \in A, a_{i} \in$ $A_{i}, b \in B, b_{j} \in B_{j}$.

Seja $V=V_{0}+V_{1}$ um superespaço vetorial, e $f(x, y)$ uma forma bilinear supersimétrica em $V$. Seja $J=V \dot{+} \mathbb{F} \cdot 1$ com multiplicação dada por

$$
(v+\alpha \cdot 1)(w+\beta \cdot 1)=(\alpha w+\beta v)+(\alpha \beta+f(v, w)) \cdot 1
$$

Assim, $J$ é uma superálgebra com graduação dada por $A=V_{0}+\mathbb{F} \cdot 1, M=V_{1}$.

Proposição 4.9 Sejam $V=V_{0}+V_{1}$ é um superespaço vetorial de dimensão maior que 1 e $f(x, y)$ uma forma bilinear supersimétrica não degenerada. Então $J(V, f)$ é uma superálgebra de Jordan especial e simples.

Demonstração: $\quad$ Seja $J=J\left(V_{0}+V_{1}, f\right) \operatorname{com} \operatorname{dim} V_{0}=n \operatorname{e~} \operatorname{dim} V_{1}=2 m$.

Vamos escrever $V_{0}=\sum_{i=o}^{n} \mathbb{F} \cdot v_{i}$ e $V_{1}=\sum_{i=0}^{2 m} \mathbb{F} \cdot u_{i}$ definamos $f\left(v_{i}, v_{j}\right)=$ $\alpha_{i} \delta_{i j}, f\left(u_{i}, u_{j+m}\right)=\delta_{i j}$ para $1 \leq i \leq j \leq m$, e $f\left(u_{i}, u_{k}\right)=0$ para $i, k \leq m$ ou $i, k>m$.

Sejam Cl $\left(\alpha_{1}, \ldots, \alpha_{n}\right)=\operatorname{alg}\left\langle v_{1}, \cdots, v_{n}\right\rangle$ com $v_{i}^{2}=\alpha_{i}$ a superálgebra de Clifford e $W_{m}=\operatorname{alg}\left\langle u_{1}, \ldots, u_{m} ; u_{m+1}, \ldots, u_{2 m}\right\rangle$ definidas em 3.3. Consideremos a graduação $\bar{u}_{i}=\bar{v}_{j}=1$.

Construímos a superálgebra associativa $U=\mathrm{Cl}\left(\alpha_{1}, \ldots, \alpha_{n}\right) \widetilde{\otimes} W_{m}$. Como $\left(v_{i} \widetilde{\otimes} u_{j}\right)=\left(v_{i} \widetilde{\otimes} 1\right)\left(1 \widetilde{\otimes} u_{j}\right)$, temos então que

$$
U=\operatorname{alg}\left\langle v_{1} \otimes 1, \ldots, v_{n} \otimes 1,1 \otimes u_{1}, \ldots, 1 \otimes u_{2 m}\right\rangle
$$

Denotamos $\left(1 \widetilde{\otimes} u_{i}\right)$ por $\widetilde{u}_{i}$ e $\left(v_{j} \widetilde{\otimes} 1\right)$ por $\widetilde{v}_{j}$. A graduação em $U$ é dada por $\overline{\widetilde{u_{i}}}=1$ e $\overline{\widetilde{v}_{i}}=0$. Dada a associatividade em $U$, temos que $U^{+s}$ é uma superálgebra de Jordan. 
Seja $\phi: J(V, f) \longrightarrow U^{+s}$ tal que $\phi\left(v_{i}\right)=v_{i} \widetilde{\otimes} 1, \phi\left(u_{j}\right)=1 \widetilde{\otimes} u_{j}$.

1. Pela construção da superálgebra $U$ e a definição da aplicação $\phi$, é claro que $\phi(A) \subseteq U_{0}$ e $\phi(M) \subseteq U_{1}$.

2. Vejamos agora que é homomorfismo de álgebras. Sejam $v_{i}, v_{j} \in V_{0} \mathrm{e}$ $u_{t}, u_{s} \in V_{1}$, pela definição de multiplicação em $J(V, f)$ temos o seguinte:

$$
\begin{aligned}
v_{i} v_{j}=f\left(v_{i}, v_{j}\right) & =\left\{\begin{array}{lll}
\alpha_{i} & \text { Se } & i=j \\
0 & \text { Se } & i \neq j
\end{array}\right. \\
{\left[u_{s}, u_{t}\right]=f\left(u_{s}, u_{t}\right) } & =\left\{\begin{array}{lll}
\delta_{s j} & \text { Se } t=m+j, & t \leq s \leq j \leq m \\
0 & \text { Se } & s, t \geq m, \quad s, t \leq m .
\end{array}\right. \\
u_{i} \cdot v_{j}=f\left(u_{i}, v_{j}\right) & =0
\end{aligned}
$$

Consideremos $\widetilde{v}_{i}, \widetilde{v}_{j} \in U_{0}$ e $\widetilde{u}_{s}, \widetilde{u}_{t} \in U_{1}$. Lembramos que na álgebra de Clifford $v_{i} v_{j}=0$ se $i \neq j$ e $v_{i}^{2}=\alpha_{i}$, e na álgebra $W_{m}, u_{i} u_{j}=$ $u_{j} u_{i}$ para $i, j \leq m$ ou $i, j \geq m+1$ e $u_{i} u_{m+j}-u_{m+j} u_{i}=\delta_{i j}$ para $i, j=1, \ldots, m$. Pelas propriedades do produto tensorial torcido e as observações anteriores temos:

$$
\begin{aligned}
& \widetilde{v_{i}} \tilde{v}_{j}=\left(v_{i} \widetilde{\otimes} 1\right)\left(v_{j} \widetilde{\otimes} 1\right)= \begin{cases}\left(\alpha_{i} \widetilde{\otimes} 1\right) & \text { Se } i=j \\
0 & \text { Se } i \neq j\end{cases} \\
& {\left[\tilde{u}_{s}, \tilde{u}_{t}\right]=\left(1 \widetilde{\otimes} u_{s}\right)\left(1 \widetilde{\otimes} u_{t}\right)= \begin{cases}\left(1 \widetilde{\otimes} \delta_{s j}\right) & \text { Se } t=m+j, \quad 1 \leq s \leq j \leq m \\
0 & \text { Se } \quad s, t \geq m, \quad s, t \leq m\end{cases} }
\end{aligned}
$$

Das observações em (4.3), (4.4) e considerando o produto supersimétrico "os" em $U$ temos:

$$
\begin{aligned}
& \phi\left(v_{i}\right) \circ_{s} \phi\left(v_{j}\right)=\left(v_{i} \widetilde{\otimes} 1\right) \circ_{s}\left(v_{j} \widetilde{\otimes} 1\right)=\frac{1}{2}\left[\left(v_{i} \widetilde{\otimes} 1\right)\left(v_{j} \widetilde{\otimes} 1\right)+\left(v_{j} \widetilde{\otimes} 1\right)\left(v_{i} \widetilde{\otimes} 1\right)\right]= \\
& \frac{1}{2}\left[\left(v_{i} v_{j} \widetilde{\otimes} 1\right)+\left(v_{j} v_{i} \widetilde{\otimes} 1\right)\right]= \begin{cases}\frac{1}{2} 2\left(v_{i}{ }^{2} \widetilde{\otimes} 1\right) & \text { Se } i=j \\
\frac{1}{2}\left(v_{i} v_{j}+v_{j} v_{i} \widetilde{\otimes} 1\right) & \text { Se } i \neq j\end{cases}
\end{aligned}
$$


Isto é: $\phi\left(v_{i}\right) \circ_{s} \phi\left(v_{j}\right)=\left\{\begin{array}{ll}\left(\alpha_{i} \widetilde{\otimes} 1\right) & \text { Se } i=j \\ 0 & \text { Se } i \neq j\end{array}=\phi\left(v_{i} v_{j}\right)\right.$.

Por um raciocínio análogo se verifica $\phi\left(u_{i} \hat{u}_{j}\right)=\phi\left(f\left(u_{i}, u_{j}\right)\right)=\phi\left(u_{i}\right) \circ_{s}$ $\phi\left(u_{j}\right), \phi\left(v_{i} u_{j}\right)=\phi\left(f\left(v_{i}, u_{j}\right)\right)=\phi(0)=0$. Além disso, temos que $\operatorname{Ker} \phi=0$.

\section{4.) Superálgebra de Kaplansky $K_{3}$}

Seja $K_{3}=\mathbb{F} e \dot{+}(\mathbb{F} x+\mathbb{F} y)$ com produto dado por $e^{2}=e, e \cdot x=\frac{1}{2} x, e \cdot y=\frac{1}{2} y$ $\mathrm{e}[x, y]=e$.

$K_{3}$ é dita a superálgebra de Kaplansky, tambem denotada por Kap, ela tem a propriedade de ser a única superálgebra de Jordan simples de dimensão finita que não possui unidade.

Proposição 4.10 A superálgebra $K_{3}$ é uma superálgebra de Jordan especial.

Demonstração: Seja $B=M(1 \mid 1)\left(W_{1}\right)$ a álgebra de matrizes $2 \times 2 \mathrm{com}$ entradas na álgebra de Weyl $W_{1}=\operatorname{alg}\langle\gamma, \eta: \gamma \eta-\eta \gamma=1\rangle$, e seja a aplicação $\phi: K_{3} \longrightarrow B^{+s}$ tal que $e \mapsto\left(\begin{array}{ll}1 & 0 \\ 0 & 0\end{array}\right), x \mapsto 2\left(\begin{array}{ll}0 & 1 \\ \gamma & 0\end{array}\right), y \mapsto\left(\begin{array}{ll}0 & \eta \\ \gamma \eta & 0\end{array}\right)$.

Vamos verificar que $\phi$ é um homomorfismo de superálgebras.

1. Pelas propriedades das matrices temos $\phi$ é linear. Pela definição das imagens de $\phi$ e as propriedades da superálgebra $M(2,2)$ é claro que $\phi(A) \subseteq(M(2,2))_{0}$ e $\phi(M) \subseteq(M(2,2))_{1}$.

2. Vejamos agora que $\phi$ é homomorfismo de álgebras. Para isso, vamos só considerar $\phi(e x), \phi(e y), \phi([x, y])$.

$$
\phi(e x)=\phi\left(\frac{1}{2} x\right)=\frac{1}{2} \phi(x)=\left(\begin{array}{cc}
0 & 1 \\
\gamma & 0
\end{array}\right)
$$




$$
\begin{gathered}
\phi(e y)=\phi\left(\frac{1}{2} y\right)=\frac{1}{2} \phi(y)=\frac{1}{2}\left(\begin{array}{cc}
0 & \eta \\
\gamma \eta & 0
\end{array}\right) \\
\phi([x, y])=\phi(e)=\left(\begin{array}{ll}
1 & 0 \\
0 & 0
\end{array}\right)
\end{gathered}
$$

Por outro lado temos

$$
\begin{gathered}
\phi(e) \circ_{s} \phi(x)=\left(\begin{array}{ll}
1 & 0 \\
0 & 0
\end{array}\right) \circ_{s} 2\left(\begin{array}{ll}
0 & 1 \\
\gamma & 0
\end{array}\right)=\left(\begin{array}{ll}
1 & 0 \\
0 & 0
\end{array}\right)\left(\begin{array}{ll}
0 & 1 \\
\gamma & 0
\end{array}\right) \\
+\left(\begin{array}{ll}
0 & 1 \\
\gamma & 0
\end{array}\right)\left(\begin{array}{ll}
1 & 0 \\
0 & 0
\end{array}\right)=\left(\begin{array}{ll}
0 & 1 \\
0 & 0
\end{array}\right)+\left(\begin{array}{ll}
0 & 0 \\
\gamma & 0
\end{array}\right)=\left(\begin{array}{ll}
0 & 1 \\
\gamma & 0
\end{array}\right)=\phi(e x)
\end{gathered}
$$

De um modo análogo se prova que $\phi(e y)=\phi(e) \phi(y)$. Só resta verificar que $\phi([x, y])=\phi(x) \circ_{s} \phi(y)$.

$$
\begin{aligned}
\phi(x) \circ_{s} \phi(y) & =2\left(\begin{array}{cc}
0 & 1 \\
\gamma & 0
\end{array}\right) \circ_{s}\left(\begin{array}{cc}
0 & \eta \\
\gamma \eta & 0
\end{array}\right)=\left(\begin{array}{cc}
0 & 1 \\
\gamma & 0
\end{array}\right)\left(\begin{array}{cc}
0 & \eta \\
\gamma \eta & 0
\end{array}\right) \\
& +\left(\begin{array}{cc}
0 & \eta \\
\gamma \eta & 0
\end{array}\right)\left(\begin{array}{ll}
0 & 1 \\
\gamma & 0
\end{array}\right)=\left(\begin{array}{cc}
\gamma \eta & 0 \\
0 & \gamma \eta
\end{array}\right)-\left(\begin{array}{cc}
\eta \gamma & 0 \\
0 & \gamma \eta
\end{array}\right) \\
& =\left(\begin{array}{cc}
\gamma \eta-\eta \gamma & 0 \\
0 & 0
\end{array}\right)=\left(\begin{array}{ll}
1 & 0 \\
0 & 0
\end{array}\right)=\phi(e)=\phi([x, y]) .
\end{aligned}
$$

Note que Ker $\phi=0$.

\section{5.) Superálgebra $D_{\tau}$}

Seja $D_{\tau}=\left(\mathbb{F} e_{1}+\mathbb{F} e_{2}\right) \dot{+}(\mathbb{F} x+\mathbb{F} y)$ com multiplicação dada por $e_{i}^{2}=e_{i}, \quad e_{1} e_{2}=0 \quad e_{i} x=\frac{1}{2} x, \quad e_{i} y=\frac{1}{2} y, \quad[x, y]=e_{1}+\tau e_{2}, \quad \tau \in \mathbb{F}$.

Para $\tau$ diferente de 0 temos que $D_{\tau}$ é simples, e reciprocamente. 
Proposição 4.11 A superálgebra $D_{\tau}$ é uma superálgebra de Jordan especial.

Demonstração: $\quad$ Seja $B=M(1 \mid 1)\left(W_{1}\right)$ a álgebra de matrizes $2 \times 2 \mathrm{com}$ entradas na álgebra de Weyl $W_{1}=\operatorname{alg}\langle\gamma, \eta: \gamma \eta-\eta \gamma=1\rangle$, e seja a aplicação $\phi: \nabla_{t} \longrightarrow B^{+s}$ tal que $e_{1} \mapsto\left(\begin{array}{ll}1 & 0 \\ 0 & 0\end{array}\right), e_{2} \mapsto\left(\begin{array}{ll}0 & 0 \\ 0 & 1\end{array}\right) x \mapsto 2\left(\begin{array}{ll}0 & 1 \\ \gamma & 0\end{array}\right)$, $y \mapsto\left(\begin{array}{cc}0 & \eta \\ \gamma \eta & 0\end{array}\right)$.

Para verificar que $\phi$ é um homomorfismo de superálgebras procedemos de um modo análogo à prova da Proposição 4.10.

\section{6.) Superálgebra de Kac $K_{10}$}

Seja $M=\mathbb{F} \cdot x+\mathbb{F} y+\mathbb{F} \cdot w+\mathbb{F} \cdot v, \quad A=A_{1} \dot{+} A_{2}$ onde

$A_{1}=\mathbb{F} \cdot e_{1}+\mathbb{F} \cdot w x+\mathbb{F} \cdot w y+\mathbb{F} \cdot v x+\mathbb{F} \cdot v y$ e $A_{2}=\mathbb{F} e_{2}$ com multiplicação definida por

$$
\begin{aligned}
& e_{i}^{2}=e_{i}, e_{1} \text { é a unidade de } A_{1} . \\
& e_{i} m=\frac{1}{2} m \quad \forall m \in M \\
& {[w, x]=w x, \quad[w, y]=w y \quad[v, x]=v x \quad[v, y]=v y} \\
& {[x, y]=e_{1}-3 e_{2}, \quad[w, x] \cdot y=-w, \quad[v, x] \cdot y=-v} \\
& {[w x, v y]=2 e_{1}}
\end{aligned}
$$

e qualquer outra multiplicação não nula é obtida da lista anterior por aplicação de qualquer uma das anti-simétrias $x \leftrightarrow y, v \leftrightarrow w$ ou das substituições simultâneas $x \leftrightarrow v, y \leftrightarrow w$.

Se o corpo $\mathbb{F}$ tem característica diferente de 3 , então $K_{10}$ é simples, caso contrario temos que $K_{10}$ não é simples embora contem um ideal simples $K_{9}=A_{1}+M$ de dimensão 9 .

$K_{9}$ é chamada superálgebra degenerada de Kiac. 


\section{7.) Superálgebra de colchetes de Poisson-Grassmann}

Seja $\Gamma$ a álgebra de Grassmann nas variáveis $e_{1}, \ldots e_{n} \ldots$ Nos vimos no Capítulo 3, que $\Gamma$ é uma superálgebra associativa, comutativa. Consideremos o colchete $\{$,$\} em \Gamma$ dada por:

$$
\left\{e_{i}, e_{j}\right\}=\left\{\begin{array}{cc}
-1, & i=j \\
0, & i \neq j
\end{array} \quad\{a b, c\}=a\{b, c\}+(-1)^{\bar{a} \bar{b}}\{a, c\} b .\right.
$$

Dados $f, g \in \Gamma_{0} \cup \Gamma_{1}$,

$$
\{f, g\}=(-1)^{\bar{f}} \sum_{j} \frac{\partial f}{\partial e_{j}} \frac{\partial g}{\partial e_{j}} \quad f, g \in \Gamma_{0}, \Gamma_{1}
$$

onde

$$
\frac{\partial}{\partial e_{j}}\left(e_{i_{1}} e_{i_{2}} \cdots e_{i_{n}}\right)= \begin{cases}(-1)^{k-1} e_{i_{1}} e_{i_{2}} \cdots e_{i_{k-1}} e_{i_{k+1}} \cdots e_{i_{n}}, & \text { se } j=i_{k} \\ 0, & \text { se } j \notin\left\{i_{1}, \ldots i_{n}\right\} .\end{cases}
$$

Note que $\{1, f\}=0, \quad \forall f \in \Gamma_{0} \cup \Gamma_{1}, \mathrm{e}\langle\Gamma,+,\{\}$,$\rangle é uma álgebra de Lie,$ portanto, o colchete é um colchete de Poisson.

O produto colchete descrito acima é dito colchete de Poisson-Grassmann.

Assim, temos a superálgebra de colchetes $J(\Gamma)=\Gamma+\Gamma \cdot u$. Como vimos anteriormente $J(\Gamma)$ é uma superálgebra com componentes $J(\Gamma)_{0}=\Gamma_{0}+\Gamma_{1} \cdot u$ e $J(\Gamma)_{1}=\Gamma_{1}+\Gamma_{0} \cdot u$.

Seja $\Gamma_{n}$ a subsuperálgebra de $\Gamma$ gerada por $e_{1}, \cdots e_{n}$. Denotamos por $J\left(\Gamma_{n}\right)$ a subsuperálgebra $\Gamma_{n}+\Gamma_{n} \cdot u$ de $J(\Gamma)$.

Proposição 4.12 Para todo $n>1$ a superálgebra $J\left(\Gamma_{n}\right)$ é simples.

Demonstração: Seja $I$ um ideal de $J\left(\Gamma_{n}\right)$. Se $0 \neq i=g+h u \in I$, $g \in \Gamma_{j}, h \in \Gamma_{1-j}, g=\sum g_{i}, h=\sum h_{j}$ então existem $e_{i_{1}}, \ldots, e_{i_{r}}$ tais que, para $f=e_{i_{1}} \cdots e_{i_{r}}$, verifica-se o seguinte:

$$
g f \neq 0 \text { e } h f=0 \quad \text { ou } \quad g f=0 \text { e } h f \neq 0 .
$$


(para observar isso, inicialmente reduzimos para o caso em que $g$ é um monômio, resultando em 2 casos: quando também podemos reduzir $h$ a um monômio, ou $h f=0$ ) Podemos supor que $g \in I$ ou gu $\in I$, onde $g \neq 0$ é um monômio de $\Gamma$. Assim, $g u \in I$, onde $g \neq 0$ é um monômio de $\Gamma$. Seja $\Gamma=g_{1} e_{m}$. Temos que $\pm g_{1}=\left\{g, e_{m}\right\}=g u \cdot e_{m} u \in I$. Portanto $g_{1} u \in I$. Procedendo com esse raciocínio, obtemos que $1 \in I$, conseqüentemente, $I \in J\left(\Gamma_{n}\right)$.

Estamos agora em condições de enunciar o primeiro teorema de classificação de superálgebras de Jordan simples de dimensão finita, dado por Kac 1977, [6], e completado por Kantor 1989, [8].

Teorema 4.13 (Kac, Kantor) Cada superálgebra de Jordan simples de dimensão finita sobre um corpo $\mathbb{F}$ algebricamente fechado de característica zero é isomorfa a uma das seguintes superálgebras

1. $M(n \mid m)^{+s}$.

2. $M_{n}[u]^{+s}, \quad n>1$.

3. $\operatorname{Osp}(n \mid 2 k)$.

4. $\operatorname{trp}(n \mid n), \quad n>1$.

5. $J(V, f)$.

6. $K_{3}$.

7. $D_{\tau}, \quad \tau \neq 0$.

8. $K_{10}$.

9. $J\left(\Gamma_{n}\right)$.

Onde as superálgebras de (1)-(7) são especiais. 


\subsection{Em corpos de característica prima $p$}

\subsubsection{Parte par de $J=A+M$ semi-simples}

Vamos considerar as superálgebras do tipo $H\left(B_{3}\right)$, onde $B$ é uma superálgebra alternativa com superinvolução $*$ e tal que os elementos $*$-simétricos pertencem ao centro de $B$.

O espaço $H\left(B_{3}\right)$ das matrizes *-hermitianas $3 \times 3$ sobre $B$ é uma superálgebra de Jordan sob a multiplicação de matrizes supersimétrica. Se $B$ é simples e não associativa, então $H\left(B_{3}\right)$ é simples e excepcional.

Consideremos os dois exemplos seguintes, ambas construções são dadas por Shestakov, [17].

1. $\mathrm{B}(1,2)$

Seja $A_{3}=J(V, f)$ a superálgebra de Jordan de superforma bilinear clada. na Proposição 4.9, com $\operatorname{dim} V_{0}=0, \operatorname{dim} V_{1}=2$ e $f(x, y)=x y$. As$\operatorname{sim} A_{3}=\mathbb{F}+\mathbb{F} x+\mathbb{F} y$ com multiplicação $[x, y]=-[y, x]=1 . A_{3}$ é uma superálgebra alternativa sobre corpos de característica três, [17], é denotada por $B(1,2)$. Considere a superinvolução em $A_{3}$ dada por $\overline{(\alpha \cdot e+x)}=\alpha \cdot e-x \operatorname{com} H\left(A_{3},-\right)=\mathbb{F} e$. Seja $*=^{-t}$ a involução em $H\left(A_{3}\right)$ induzida por - Como $A_{3}$ é simples e não associativa, então $H\left(A_{3}, *\right)$ é uma superálgebra de Jordan simples e excepcional de dimensão 12 .

2. $\mathrm{B}(4,2)$

Seja $B=M_{2}(\mathbb{F})+\left(\mathbb{F} m_{1}+\mathbb{F} m_{2}\right)$. A multiplicação em $M_{2}(\mathbb{F})$ é conhecida. 
As outras multiplicações não nulas estão dadas por

$$
\begin{array}{llll}
e_{11} m_{1}=m_{1}, & e_{22} m_{1}=0, & e_{12} m_{1}=m_{2}, & e_{21} m_{1}=0 . \\
m_{1} e_{11}=0, & m_{1} e_{22}=m_{1}, & m_{1} e_{12}=-m_{2}, & m_{1} e_{21}=0 . \\
e_{11} m_{2}=0, & e_{22} m_{2}=m_{2}, & e_{12} m_{2}=0, & e_{21} m_{2}=m_{1} . \\
m_{2} e_{11}=m_{2}, & m_{2} e_{22}=0, & m_{2} e_{12}=0, & m_{1} e_{21}=-m_{1} . \\
{\left[m_{1}, m_{1}\right]=-e_{21},} & {\left[m_{2}, m_{2}\right]=e_{12},} & {\left[m_{1}, m_{2}\right]=e_{11},} & {\left[m_{2}, m_{1}\right]=-e_{22} .}
\end{array}
$$

Sobre um corpo $\mathbb{F}$ de característica três $B$ é uma superálgebra alternativa com superinvolução $(\alpha+m)^{*}=\bar{\alpha}-m$, onde - é a involução simplética de $M_{2}(\mathbb{F})$. Então, $H_{3}(B)$ o conjunto das matrizes simétricas com respeito à superinvolução *-transposta é uma superálgebra de Jordan simples a qual é excepcional, pois $B$ não é associativa. A superálgebra $B$ é denotada por $B(4,2)$ e tem dimensão 21 .

Estamos agora em condições de enunciar o segundo teorema de classificação de superálgebras de Jordan de dimensão finita simples, demonstrado por Racine e Zelmanov, [11].

Teorema 4.14 (Racine, Zelmanov) Seja $J=A+M$ uma superálgebra de Jordan simples de dimensão finita sobre um corpo algebricamente fechado de característica diferente de dois. Se A é semi-simples e $M \neq\{0\}$ então $J$ é isomorfa a uma das seguintes superálgebras.

1. $M(n \mid m)^{+s}$.

2. $M_{n}[u]^{+s}, \quad n>1$.

3. $\operatorname{Osp}(n \mid 2 k)$.

4. $\operatorname{trp}(n \mid n), \quad n>1$.

5. $J(V, f)$.

6. $K_{3}$. 
7. $D_{\tau}, \quad \tau \neq 0$.

8. $K_{10}$.

9. $K_{9}$ superálgebra degenerada de Kac, quando $\operatorname{Car} \mathbb{F}=3$.

10. $H_{3}(B)$ quando Car $\mathbb{F}=3$, onde $B=B(1,2)$ ou $B=B(4,2)$.

\subsubsection{Parte par de $J=A \dot{+} M$ não é semi-simples}

1. Superálgebra $O(m, n)$

Seja $\mathbb{F}$ um corpo algebricamente fechado de característica $p>2$. Sejam $\Gamma_{n}$ a álgebra de Grassmann em $n$-variáveis e $O(m)=\mathbb{F}\left[a_{1}, a_{2}, \ldots, a_{m} \mid\right.$ $a_{1}^{p}=a_{2}^{p}=\cdots=a_{m}^{p}=0$ ] a álgebra de polinômios truncados. Consideremos a superálgebra produto tensorial $O(m, n)$ dada por

$$
O(m, n)=O(m) \otimes_{\mathbb{F}} \Gamma_{n}=O(m) \otimes_{\mathbb{F}}\left(\Gamma_{n}\right)_{0}+O(m) \otimes_{\mathbb{F}}\left(\Gamma_{n}\right)_{1}
$$

A superálgebra $O(m, n)$ é uma superálgebra associativa e comutativa.

\section{Superálgebra Cheng-Kac}

Seja $Z$ uma álgebra comutativa, associativa com unidade e derivação $d$ : $Z \longrightarrow Z$. Consideremos a superálgebra $\operatorname{CK}(Z, d)=A+M$, onde $A=$ $Z+\sum_{i=1}^{3} w_{i} Z, M=x Z+\sum_{i=1}^{3} x_{i} Z$ são $Z$-módulos de posto 4 . A multiplicação em $A$ é $Z$-linear e $w_{i} w_{j}=0, i \neq j$ e $w_{1}{ }^{2}=w_{2}{ }^{2}=1, w_{3}{ }^{2}=$ -1 .

Denotemos $x_{i \times i}=0, x_{1 \times 2}=-x_{2 \times 1}=x_{3}, x_{1 \times 3}=-x_{3 \times 1}=x_{2},-x_{2 \times 3}=$ 
$x_{3 \times 2}=x_{1}$. A estrutura de bimódulo $A \times M \longrightarrow M$ é definida via:

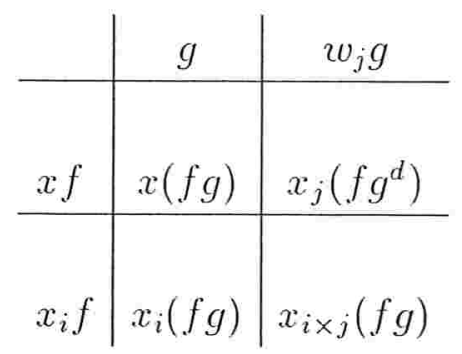

O colchete em $M$ é definido via:

\begin{tabular}{c|c|c} 
& $x g$ & $x_{j} g$ \\
\hline & & \\
$x f$ & $f^{d} g-f g^{d}$ & $-w_{j}(f g)$ \\
\hline & & \\
$x_{i} f$ & $w_{i}(f g)$ & 0
\end{tabular}

Vamos verificar que a multiplicação dada define uma superálgebra de Jordan.

Teorema $4.15 \mathrm{CK}(Z, d)$ é uma superálgebra de Jordan

Demonstração: Para uma demonstração deste resultado ver [13].

Lema $4.16 \mathrm{CK}(Z, d)$ é simples se, e somente se, $Z$ não contém ideais próprios d-invariantes.

Demonstração: $\quad$ Se $I$ é um ideal $d$-invariante de $Z$, então $I+\sum_{i=1}^{3} w_{i} I+$ $x I+\sum_{i=1}^{3} x_{i} I+x I$ é um ideal de $\mathrm{CK}(Z, d)$.

Reciprocamente, suponhamos que $B$ é um ideal de $\operatorname{CK}(Z, d)$. Se $B \cap Z \neq$ $\{0\}$ então $B \cap Z$ é um ideal $d$-invariante de $Z$. Assim, $Z \subset B$, e como $1 \in Z$, segue se que $B=\operatorname{CK}(Z, d)$. Suponhamos que $B \cap Z=\{0\}$. Logo qualquer ideal não nulo de $A$ encontra $Z$, segue se que $B \cap Z=\{0\}$. Portanto $[B, M]=\{0\}$. Seja $b \in B$ tal que $b=x z_{0}+\sum_{i=1}{ }^{3} x_{i} z_{i}$. Mas nós temos que 
$\left[b, x_{1}\right]=-w_{1} z_{0} \in B \cap A$. Assim, $z_{0}=0$. Agora, $[b, x]=\sum_{i=1}{ }^{3} w_{i} z_{i} \in B \cap A$. Portanto, $z_{i}=0$ para $1 \leq i \leq 3$.

Estamos finalmente em condições de enunciar o nosso último teorema de classificação das superálgebras de Jordan de dimensão finita. O Teorema foi demosntrado por Martinez e Zelmanov, [12].

Teorema 4.17 (Martinez, Zelmanov) Seja $J=A+M$ uma superálgebra de Jordan simples de dimensão finita sobre um corpo algebricamente fechado e de característica prima $p>2$ e tal que A não é semi-simples. Então, J é isomorfa a uma das seguintes superálgebras:

1. Existem inteiros $m, n$ e um colchete de Jordan $\{$,$\} em O(m, n)$ tal que $J=O(m, n)+O(m, n) x$ é uma superálgebra dobrada de Kantor.

2. Superálgebra de Jordan Cheng-Kac, $\mathrm{CK}(B(m), d)$ correspondente a uma derivação d $: B(m) \longrightarrow B(m)$.

Em [13], foi demonstrado para $n \geq 2$ que as superálgebras simples $J(O(m, n),\{\}$, são excepcionais, e a superálgebra de Jordan Cheng-Kac é especial.

\subsection{Algumas observações}

Algumas diferenças estruturais entre álgebras e superálgebras de Jordan

Como continuação vamos mencionar alguns resultados que nos permitem ver diferencias entre as álgebras de Jordan e as superálgebras de Jordan.

1. Primeiramente, o radical solúvel Solv $J$ em superálgebras de Jordan pode não ser nilpotente, mesmo assim com dimensão finita como se prova em [18]. 
2. A superálgebra semi-simples não necessariamente é uma soma direita de subsuperálgebras simples. Um exemplo disto é dada pela superálgebra com unidade $\operatorname{kap}_{3}^{\#}$.

3. Ainda está em aberto o problema de validade de um análogo ao Teorema de Wedderburn para o caso de superálgebras de Jordan. Isto é, dada uma superálgebra de Jordan de dimensão finita, e Solv $J$ seu radical, existe uma subsuperálgebra semi-simples $B$ de $J$ tal que $J /$ Solv $J$ é isomorfa $\operatorname{com} B$ e $J=B+\operatorname{Solv} J$

O nosso objetivo num seguinte trabalho, o qual pretendemos desenvolver no doutorado é estudar a validade do teorema de Wedderburn para o caso de superálgebras de Jordan de dimensão finita. 


\section{Referências Bibliográficas}

[1] R.D. Schafer., An introduction to nonassociative algebras, Academic Press, N.Y. (1966)

[2] N. Jacobson, Structure and representation of Jordan algebras; American Mathematical Society, Colloquium Publications, vol XXXIX (1968).

[3] K. Zhevlakov, I. Shestakov, A. Slin'ko, A. Shirshov, Rings that are nearly associative, Academic Press, N.Y. 1982.

[4] E.N. Kuzmin, and I.P. Shestakov, Nonassociative structures; VINITI, Itogi nauki i tekhniki, seria "Fundamental Branches", v.57, 179-266, Moskow, 1990; English transl. in "Encyclopedia of Math. Sciences, v.57, Algebra VI, 199-280" edited by A.I.Korstrikin and I.R. Shafarevich, Springer-Verlag.

[5] K. McCrimmon, Taste of Jordan Algebra, Springer Verlag, 2003

[6] V.G. Kac, Classification of simple $\mathbb{Z}$-graded Lie superalgebras and simple Jordan superalgebras; Comunications in algebra, 5(13),1375-1400 (1977).

[7] C. Ambrosi Gómez, On the simplicity of hermitian superalgebras, Nova J. Algebra Geometry. (1995) no. 3, 193-198. 
[8] I.L. Kantor, Connections between Poisson brackets and Jordan Lie superalgebras, Lie theory, diferential equations and representation theory, 213-225, Montreal.(1989)

[9] K. McCrimmon, Speciality and non-speciality of two Jordan superalgebras, J. of Algebra 149,(1992) no. 2, 326-351.

[10] D. King and K. McCrimmon, The Kantor constrution of Jordan superalgebras, Comm. in Algebra (1992) 20(1), 1009-126.

[11] M.L. Racine, and E.I. Zelmanov, Simple Jordan Superalgebras with semisimple even part, J. Algebra 270, no.2, 374-444 (2003).

[12] C. Martinez, and E.I. Zelmanov, Simple finite dimensional Jordan Superalgebras of prime characterisc, J. Algebra 236 no.2, 575-629.(2001)

[13] C. Martinez, I. Shestakov, E,Zelmanov, Jordan superalgebras defined by brackets. J. London Math. Soc. 264 (2001) no.2 357-368.

[14] V. G. Kac, C. Martinez e E. Zelmanov, Graded simple Jordan superalgebras of growth one, Mem. AMS Soc, 150 (2001), no.711.

[15] I.P. Shestakov, and E.I. Zelmanov, Prime Alternative and superalgebras and nilpotence of the radical of a free alternative algebra; izvetiya, 54 (1990), Math. URRS izvestiya, 37 (1991) 19-36.

[16] I.P. Shestakov, Alternative and Jordan superalgebras; Siberian Advances in Mathematics, (1999), v.9, no.2, 83-99.

[17] I.P. Shestakov, Prime alternative superlagebras of arbitrary characteristic, (Russian) Algebra i logica 36 (1997), n0. 6, 675-716, 722; traslation in Algebra and Logic 36 (1997) no. 6. 389-412. 
[18] I. P. Shestakov, Superalgebras and Counterexamples, Sibirsk. Matem, Zhurnal 32 (1991), no. 6, 187-196; English transl. in Siberian Math. J. 32 (1991), n0. 6, 1052-1060 (1992).

[19] I.P. Shestakov, Notas de aula Superalgebras; Instituto de Matematicas e Estatistica Universidade de São Paulo, (1999). 